Aus der Klinik für Mund-, Kiefer- und Gesichtschirurgie

(Prof. Dr. med. Dr. med. dent. H. Schliephake)

im Zentrum Zahn-, Mund- und Kieferheilkunde

der Medizinischen Fakultät der Universität Göttingen

\title{
Vergleichende Untersuchung unterschiedlicher Markersysteme für die präimplantologische Röntgendiagnostik mit dem Panoramaschichtgerät
}

INAUGURAL - DISSERTATION

zur Erlangung des Doktorgrades

für Zahnheilkunde

der Medizinischen Fakultät der

Georg-August-Universität zu Göttingen

vorgelegt von

Stephan Lorenz

aus

Elsterwerda

Göttingen 2016 
Dekan:

I. Berichterstatter/in:

II. Berichterstatter/in:

III. Berichterstatter/in:
Prof. Dr. rer. nat. H. K. Kroemer

Prof. Dr. med. dent. H. Visser

Prof. Dr. med. Jan Menke

Prof. Dr. hum. biol. Margarete Schön

Tag der mündlichen Prüfung: 27.07.2016 


\section{Inhaltsverzeichnis}

1 Einleitung $\quad 1$

1.2 Präimplantologische Diagnostik 2

1.3 Planungsschablone $\quad 4$

1.4 Bildgebende Verfahren $\quad 5$

1.4.1 Die Panoramaschichtaufnahme 6

1.4.2 Intraorale Zahnaufnahme 8

1.4.3 Transversale Schichtaufnahme 8

1.4.4 Fernröntgen und Aufbissaufnahmen 9

1.4.5 Digitale Volumentomographie 9

1.5 Strahlenexposition in der Zahnmedizin 11

1.6 Zielsetzung 13

2 Material und Methoden $\quad 14$

2.1 Erstellen eines Röntgenmodells $\quad 14$

2.1.1 Prothetische Überlegungen 14

2.1.2 Das Röntgenmodell 14

2.1.3 Modifikationen des Grundmodells $\quad 15$

2.1.4 Röntgenschablonen und Markersysteme 19

2.1.5 Geometrische Überlegungen zu den verwendeten Markersystemen 22

2.1.6 Herstellung der Röntgenschablonen 24

2.1.7 Anbringen der Röntgenmarker 25

2.1.8 Einstellen der Marker 26

2.1.9 Erstellen der Panoramaschichtaufnahmen 26

2.2. Vergleich der verschiedenen Markersysteme 32

2.2.1 Randomisation 32

2.2.2 Versuchsablauf 32

2.2.3 Statistische Methoden 34

2.3 Vergleich Panoramaschichttechnik und digitale Volumentomographie 34

2.3.1 Erstellen einer DVT-Aufnahme 34

2.3.2 Vermessung der DVT-Aufnahme 35

$\begin{array}{ll}\text { 2.3.3 Vermessung der Panoramaschichtaufnahme } & 37\end{array}$

2.3.4 Statistische Methoden 39 
3.1 Ergebnisse für die verschiedenen Markersysteme $\quad 40$

$\begin{array}{ll}\text { 3.1.1 Messung aller Marker } & 40\end{array}$

3.1.2 Messung Knochenhöhen $\quad 45$

3.1.3 Ergebnisse nach Kalibrierung $\quad 47$

3.2 Vergleich OPG XG Plus und NewTom DVT 51

3.3 Zusammenfassung der Ergebnisse $\quad 53$

4 Diskussion $\quad 55$

4.1 Motivation zur Untersuchung $\quad 55$

4.2 Diskussion der Methodik $\quad 56$

4.2.1 Das Röntgenmodell $\quad 56$

4.2.2 Die Röntgenschablonen $\quad 56$

4.2.3 Positionierungsfehler $\quad 57$

4.2.4 Genauigkeit der radiologischen Abbildungen $\quad 59$

4.3 Diskussion der Ergebnisse $\quad 60$

4.3.1 Vergleich verschiedener Markersysteme im OPG XG Plus 60

4.3.2 Vergleich OPG XG Plus und NewTom DVT 64

4.4 Schlussfolgerungen für die klinische Anwendung 67

$\begin{array}{ll}5 \text { Zusammenfassung } & 71\end{array}$

6 Literaturverzeichnis $\quad 73$

$\begin{array}{ll}7 \text { Anhang } & 80\end{array}$

$\begin{array}{ll}\text { 7.1 Abkürzungsverzeichnis } & 80\end{array}$

$\begin{array}{ll}7.2 \text { Auswertungsbögen } & 81\end{array}$

$\begin{array}{ll}7.3 \text { Rohdaten } & 85\end{array}$

7.3.1 Versuch 1: Vergleich verschiedener Markersysteme 85

$\begin{array}{ll}\text { 7.3.2 Versuch 2: DVT vs OPG } & 91\end{array}$ 


\section{Einleitung}

Für die Diagnostik des vertikalen Knochenangebotes vor einer Implantatinsertion ist die Panoramaschichtaufnahme zusammen mit einer Messreferenz als Basisdokumentation anzusehen (NITSCHE et al. 2012; SCHULZE et al. 2013). Zusätzlich angefertigte Zahnaufnahmen, Fernröntgenseitenaufnahmen, Aufbissaufnahmen oder Aufnahmen der Nasennebenhöhlen können dem Behandler weitere Informationen über das Implantatlager geben.

Eine dreidimensionale Beurteilung des Knochenlagers mittels Computertomographie oder digitaler Volumentomographie sollte wegen der Strahlenbelastung und der Kosten-Nutzen-Relation auf spezielle Fragestellungen begrenzt bleiben (SCHULZE et al. 2013). Die Strahlenbelastung bei Verwendung der digitalen Volumentomographie ist zwar geringer als bei der Computertomographie, aber immer noch höher als bei der Panoramaschichttechnik (LUDLOW et al. 2015).

Um den Forderungen nach einer möglichst geringen Strahlenbelastung gerecht zu werden (Bundesamt für Strahlenschutz 2003, Röntgenverordnung § 2c; FRASCH et al. 2014), müssen die zweidimensionalen diagnostischen Verfahren präzise eingesetzt werden. Der Beurteilung des Implantatlagers nach Qualität und Quantität kommt dabei eine entscheidende Bedeutung zu. Dabei sollte eine optimale Ausnutzung des vorhandenen Implantatlagers gewährleistet werden, ohne die Integrität angrenzender anatomischer Strukturen wie Nasen- und Kieferhöhlenboden oder den Canalis mandibularis zu gefährden (SCHWENZER und EHRENFELD 2009).

Ein wichtiges Hilfsmittel der präimplantologischen Diagnostik sind Planungsschablonen mit Röntgenmarkern. Sie dienen der Einschätzung des vorhandenen vertikalen Knochenangebotes sowie der präzisen Planung der Implantatposition (KOEK und WAGNER 2004). Hierbei können ganz unterschiedliche Markersysteme zum Einsatz kommen. Diese unterscheiden sich in ihrer Form und der Lage zum Implantatlager. Vergleichende Untersuchungen zur Eignung der unterschiedlichen Systeme fehlen, insbesondere was Schablonen betrifft, die mit konventioneller Zahntechnik erstellt werden. 


\subsection{Präimplantologische Diagnostik}

Jeder speziellen implantologischen Diagnostik geht die zahnärztliche Befundaufnahme voraus. Diese enthält neben der allgemeinmedizinischen und zahnärztlichen Anamnese die extra- und intraorale Inspektion und Palpation sowie die Beurteilung des Funktionsstatus des Patienten. Bei Vorliegen einer Funktionsstörung muss zunächst das funktionelle Gleichgewicht wiederhergestellt werden (GUTWALD et al. 2010).

Zur speziellen implantologischen Diagnostik gehören die Beurteilung der Schleimhautsituation mit Verlauf und Ausprägung der attached gingiva, der Knochenresorption und der Kieferrelationsverhältnisse. Die bidigitale Palpation der Basis mandibulae ermöglicht dem Behandler einen Eindruck des Knochenangebotes in der Breite (NEUKAM et al. 2007). Außerdem können Konkavitäten des Unterkieferknochens in der Transversalebene erkannt werden.

Ist eine Implantation indiziert und vom Patienten gewünscht, sollten Situationsmodelle erstellt werden. Diese dienen der Planung sowie dem Erstellen einer Röntgenmessschablone unter Verwendung von röntgenopaken Referenzierungsmarkern. Eine mit dieser Planungsschablone erstellte Panoramaschichtaufnahme ermöglicht die quantitative Abschätzung des vertikalen Knochenangebotes. Zudem kann die Dichte der Knochenstruktur orientierend beurteilt werden. Sollten hier osteolytische Defekte diagnostiziert werden oder ein Verdacht auf Osteoporose entstehen, muss diesen Befunden vorrangige Beachtung gegeben werden. Dasselbe gilt bei Verdacht auf eine Eröffnung der Kieferhöhle mit Gefahr einer Sinusitis maxillaris bei entsprechender Implantationsplanung in dieser Region.

Nach einem Zahnverlust kann es durch externes und internes Remodelling zu einem Knochenabbau kommen, der zu einem progredienten Höhenverlust der Pars alveolaris und im Unterkiefer somit zu einer Annäherung des Alveolarkamms an den Mandibularkanal führt. Die Abbauvorgänge können in eine vertikale und eine horizontale Resorption eingeteilt werden (SCHWENZER und EHRENFELD 2009). Die vertikale Resorption wird durch die fehlende Zugbelastung des Parodonts auch als Inaktivitätsresorption bezeichnet. Sie ist im Unterkiefer viermal größer als im Oberkiefer. Die horizontale Resorption des Knochens ist überwiegend durch muskulären Druck von Lippen, Wange und Zunge bedingt. Insgesamt atrophiert der Oberkieferzahnbogen nach zentripetal und der Unterkiefer nach zentrifugal, was 
prothetische Probleme nach sich zieht. Durch Verkleinerung des Winkels zwischen Interalveolarkammlinien und Okklusionsebene müssen Kompromisse zwischen der möglichst axialen Krafteinleitung in den Knochen und der Konstruktion des prothetischen Aufbaus eingegangen werden.

Bei einer Implantation im Unterkiefer besteht die Gefahr der Verletzung der Integrität des Canalis alveolaris inferior (KOECK und WAGENER 2004). Dieser verläuft in der Vertikalen in einer leicht nach unten gerichteten Konvexität im Corpus mandibulae. Im Bereich des ersten Molaren ist er dem Unterrand der Mandibula am nächsten. Eine traumatische Schädigung des Nervus alveolaris inferior kann zu einer Hyp- oder Anästhesie des Unterkiefers einer Seite und in seinem Endast als Nervus mentalis zu einem dauerhaften Sensibilitätsausfall der Haut im Bereich des Kinns und der Lippe der entsprechenden Seite führen. Der Canalis mandibulae geht in den Canalis mentalis über. Dieser verläuft häufig in einem halbkreisförmigen Bogen nach bukkal, kranial und distal und liegt somit intraossär häufig weiter mesial, als es die Lage des Foramen mentale vermuten lassen würde. Daher muss bei interforaminal gesetzten Implantaten ein Sicherheitsabstand von $5 \mathrm{~mm}$ vom Foramen mentale nach mesial eingehalten werden (SCHWENZER und EHRENFELD 2009). Die vertikale verfügbare Knochenhöhe ergibt sich aus dem Abstand zwischen Kieferkamm zum Mandibularkanal minus eines Sicherheitsabstands von $2 \mathrm{~mm}$. Wenn vor der Implantatinsertion die crestale Knochenkante geglättet werden muss, um in der transversalen Dimension ein ausreichend dimensioniertes Implantatlager zu schaffen, muss dies in der Planung berücksichtigt werden. Des Weiteren muss der konkave Aufbau der Mandibula caudal der Linea mylohyoidea besonders beachtet werden (SCHWENZER und EHRENFELD 2009).

Der Oberkieferseitenzahnbereich weist bei vielen Patienten eine enge Lagebeziehung zur Kieferhöhle auf. Hier kann es zu Überlagerungen dentaler und ossärer Strukturen mit dem Kieferhöhlenboden mit seinen Underwood-Septen kommen. Eine bildgebende Diagnostik in einer zusätzlichen Ebene kann somit sinnvoll sein (CACACl et al. 2006).

Als Mindestlänge der Implantate wurden noch vor einigen Jahren $10 \mathrm{~mm}$ gefordert. Ältere Studien zu Implantatmisserfolgsraten betrafen zumeist Implantate mit gefräster Oberfläche (BUSER et al. 1997; NEDIR et al. 2004). So haben mikrostrukturierte Implantate einer Länge zwischen 6 und $10 \mathrm{~mm}$ nach aktuellem Stand der 
Wissenschaft keine signifikant höhere Misserfolgsrate als Implantate größerer Längen (KROCKOW 2012).

\subsection{Planungsschablone}

Nach der klinischen Diagnostik und Modellanalyse erfolgt die Erstellung einer Röntgenmessschablone gegebenenfalls inklusive Wax-Up, um die spätere Lage der prothetischen Suprakonstruktion von Anfang an in die Planung einzubeziehen (sogenanntes Backwards Planning). Dazu sollten bei der präimplantologischen Diagnostik stets Planungsschablonen mit Röntgenmarkern eingesetzt werden (WEBER 2010; GUTWALD et al. 2010). Das Ausrichten der Röntgenmarker erfolgt idealerweise unter Berücksichtigung ihrer Lage und Kippung zur geröntgten Schicht. Die Implantatachse sollte in der transversalen Dimension auf die tragenden Höcker der Antagonisten ausgerichtet sein - bei zahnlosem Gegenkiefer auf die Kieferkammmitte. Die sagittale Ausrichtung der Implantate wird bei regelrechter Verzahnung durch die Achse der benachbarten natürlichen Zähne vorgegeben (GERNET et al. 2011). Die Achsstellung der Implantate im Unterkieferseitenzahngebiet richtet sich

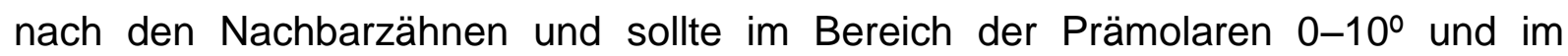
Bereich der Molaren 10-25 nach lingual gekippt sein (SCHWENZER und EHRENFELD 2009; SPIEKERMANN 1994). In dieser Region ist vor allem der Bereich kaudal der Linea mylohyoidea für eine Perforation der Kompakta gefährdet.

Die Aufstellung der zur präoperativen Planung verwendeten unterschiedlichen Marker erfolgt nach Möglichkeit in der transversalen Ebene direkt über dem Implantatlager. Bei projektionsradiographischen Verfahren werden Objekte, die bukkal und somit näher am Bildempfänger stehen, auf dem Röntgenbild kleiner abgebildet als Objekte, die lingual und somit näher an der Strahlenquelle stehen (PASLER und VISSER 2000). Objekte, die genau auf Höhe der Cresta senkrecht zum Zentralstrahl stehen, werden so mit demselben Vergrößerungsfaktor abgebildet wie der zu vermessende Knochen in der Implantatebene. Voraussetzung ist hier, dass diese Ebene auch mit der Schichtebene des Panoramaröntgengerätes übereinstimmt.

Die Röntgenmarker sollten sowohl senkrecht zur Okklusionsebene als auch senkrecht zum Zentralstrahl ausgerichtet sein. Steht der Marker nicht senkrecht zum Zentralstrahl, kann seine Projektion zu fehlerhaften Schlüssen führen. Hier haben 
kugelförmige Referenzierungsmarker den Vorteil, immer einen kippungsunabhängigen Projektionsschatten zu erzeugen.

Häufig werden Planungsschablonen so gefertigt, dass diese auch als Operationsschablonen eingesetzt werden können. Dann müssen zusätzliche Aspekte wie Führungsflächen für die entsprechenden Implantatbettbohrer, berücksichtigt werden (KOECK und WAGENER 2004). Zudem dürfen die Operationsschablonen den chirurgischen Eingriff nicht behindern und müssen intraoperativ kleine Positionskorrekturen zulassen.

Eine Studie von UHL et al. 2001, die über einen Zeitraum von 12 Monaten die Insertion von 2346 Implantaten bei 750 Patienten untersuchte, zeigte, dass oftmals die präimplantologische Diagnostik lediglich mit einem Orthopantomogramm $(54,7 \%)$, gefolgt von Orthopantomogramm mit Messkugel (40,4\%), erfolgte. Nur in wenigen Fällen wurden intraorale Zahnaufnahmen $(7,2 \%)$, Fernröntgenseitenaufnahmen $(4,8 \%)$ oder Computertomogramme $(2,2 \%)$ erstellt. Die Studie zeigte außerdem, dass für die Modellanalyse in der Mehrzahl einfache Situationsmodelle $(20,9 \%)$ hergestellt wurden, welche teilweise für die Okklusionsanalyse $(14,4 \%) \mathrm{im}$ Artikulator einartikuliert wurden. Selten wurde ein Wax-up (2\%) angefertigt. Bohrschablonen fanden in dieser Untersuchung nur in wenigen Fällen $(4,5 \%)$ Anwendung.

\subsection{Bildgebende Verfahren}

Um den Forderungen nach einer möglichst geringen Strahlenbelastung für den Patienten (Bundesamt für Strahlenschutz 2003, Röntgenverordnung § 23) nachzukommen, sollte bei der Auswahl des bildgebenden Verfahrens entschieden werden, ob bereits die zweidimensionale Panoramaschichtaufnahme zusammen mit klinischen Untersuchungsmethoden wie Palpation oder Schleimhautdickenmessung diagnostisch hinreichend sind. Zusätzliche Röntgenverfahren wie die transversale Schichtaufnahme, die intraorale Zahnaufnahme, die Computertomographie oder die digitale Volumentomographie sind mit zusätzlichen Strahlenbelastungen und erhöhten Kosten verbunden und sollten speziellen Fragestellungen vorbehalten werden. 
Die digitale Volumentomographie ist im Bereich der Implantologie der Computertomographie sowohl bezüglich der Strahlenexposition (LUDLOW und IVANOVIC 2008) als auch in der Wirtschaftlichkeit überlegen. Zudem können die Geräte in zahnärztlichen Praxen betrieben werden. Die Computertomographie hat in der Zahnmedizin eine Sonderstellung und sollte im Bereich der Zahn-MundKieferheilkunde nur bei traumatologische oder onkologische Fragestellungen indiziert sein, welche vorrangig in das Fachgebiet der Mund-Kiefer-Gesichts-Chirurgie fallen (NITSCHE et al. 2012).

\subsubsection{Die Panoramaschichtaufnahme}

Den Grundstein für die Entwicklung der Technik der Orthopantomographie legte Bocage 1921 mit der Entwicklung des Prinzips der Tomographie (PASLER 2008). Heckmann passte die Schichtaufnahmetechnik 1939 weiter an die Anatomie der Kiefer an. Schließlich führte Paatero 1949 das erste Gerät zur Produktreife (PASLER 2008). Seitdem sind große technische Fortschritte bei der Panoramaschichtaufnahme erzielt worden.

Die Panoramaschichtaufnahme bildet nicht nur die Zähne, sondern auch die Kiefer mit den benachbarten Strukturen wie den alveolären Buchten der Kieferhöhlen ab. Die Strahlenbelastung ist hierbei im Vergleich zu anderen Röntgenverfahren wie der digitalen Volumentomographie oder der Computertomographie relativ gering (NITSCHE et al. 2012).

Für die Aufnahme rotieren Röhre und Kassetten- oder Sensorträger gleichsinnig um den Gesichtsschädel. Dabei treten die Röntgenstrahlen aus einer fokusnahen Schlitzblende durch den Kopf des Patienten auf eine fokusferne vertikale Schlitzblende. Hinter dieser befindet sich der sich im Gegensinn bewegende Film mit einer Verstärkerfolie oder ein Sensor bzw. eine Speicherfolie. Die dabei entstehende „Zonographie“ (PASLER 2008) besitzt eine scharfzeichnende Schicht, die der Größe und Form des Zahnbogens angepasst werden kann. Dabei steigt die Dicke der Schicht von $9 \mathrm{~mm}$ im Frontzahnbereich bis zu ca. $20 \mathrm{~mm}$ im Bereich der Kiefergelenke (PASLER und VISSER 2003). Außerhalb der Schicht liegende anatomische Strukturen können nicht vollständig unterdrückt, sondern nur verwischt werden. Dies führt zu Summationseffekten, die fehlgedeutet werden können. 
Das Rotationszentrum ist nicht konstant, sondern wandert im Verlauf der Aufnahme von der Regio retromolaris im Gleichsinn mit dem Strahlenbündel entlang der Zahnbögen (PASLER und VISSER 2003). Außerhalb der Schicht liegende anatomische Strukturen oder Objekte werden dabei verformt abgebildet. Liegen sie vestibulär, werden sie unscharf, verschmälert und hochoval dargestellt. Oral von der Schicht liegende Objekte werden unscharf verbreitert und queroval dargestellt.

Panoramaschichtgeräte erlauben in der Regel die Schicht auf die individuellen Gegebenheiten der zu untersuchenden Person einzustellen. So können bei vielen Geräten Einstellungsmöglichkeiten zum Beispiel zwischen „Kind“, „kleine Person“ und „kräftiger Mann“ gewählt werden, um die scharfzeichnende Schicht an die Größe und Strahlendurchlässigkeit des jeweiligen Kiefers grob anzupassen.

Nachteile der Orthopantomographie sind positionsbedingte Verzerrungen, die durch nicht korrekte Platzierung des Patienten in die vorgegebene FokusProjektionsflächenanordnung des Röntgengerätes entstehen.

Häufig wird der Kopf des Patienten, wie auch von vielen Herstellern gefordert, waagerecht zur Frankfurter Horizontalen eingestellt. Diese ist definiert als Ebene des oberen Randes des Porus arcusticus externus zum tiefsten Punkt des knöchernen Randes der Orbita beider Seiten. Eine solche Einstellung des Patienten kann zu etwas verminderter Bildqualität führen (PASLER und VISSER 2003). Erst bei leichter Kippung nach hinten wird erreicht, dass der Zentralstrahl im Unterkiefer senkrecht zur Schicht verläuft, da die Frankfurter Horizontale individuell unterschiedlich einen Winkel von 10-15 Grad mit der Camperebene bildet (LEHMANN und HELLWIG 2012). SUCKERT (1992) gibt die Lage der Okklusionsebene zur Frankfurter Horizontalen mit 5,59 Grad und einer Standardabweichung von 5,73 Grad an.

Durch den kurzen Fokus-Objekt-Abstand und den relativ großen Objekt-Film-Abstand kommt es zu Vergrößerungen des Röntgenbildes um den Faktor 1:1,2 bis 1:1,35 gegenüber der tatsächlichen klinischen Situation (BEYER et al. 2011). In der horizontalen Dimension variieren die Vergrößerungsfaktoren zwischen 1:1,14 und 1:1,44 (ROMÁN et al. 2003). Auch innerhalb der Röntgenaufnahme verändert sich der Vergrößerungsfaktor, da der Fokus-Objekt- und Objekt-Filmabstand nicht konstant sind. Daher müssen Referenzierungsmarker möglichst genau an der Implantationsstelle platziert werden. Nicht berücksichtigt bleibt hierbei die Abweichung des Nervenkanals in der Horizontalebene. Auch die Implantatachse verläuft in den meisten Fällen nicht senkrecht zur Okklusionsebene. 
Die Verwendung von Mittelwertschablonen zur metrischen Auswertung von Panoramaschichtaufnahmen muss kritisch hinterfragt werden, da sie nur einen mittleren Vergrößerungsfaktor des Panoramaschichtgerätes berücksichtigen. Diese auf die Panoramaschichtaufnahme gelegten Folien zeigen Umrisse von Implantaten mit einem vorgegebenen Vergrößerungsfaktor und können somit nur Annäherungswerte vermitteln.

HÖHLER (2008) ermittelte Vergrößerungsfaktoren von 1,15 bis 1,23 bei Panoramaschichtaufnahmen. GOCH (2005) ermittelte unter Verwendung der Orthopantomograpie und der Annahme eines 1,25-fachen standardisierten Vergrößerungsfaktors Werte von 2,61 mm Abweichung zu den histologischen Werten.

\subsubsection{Intraorale Zahnaufnahme}

Zur präimplantologischen Diagnostik können intraorale Zahnaufnahmen eingesetzt werden. Bei der präimplantologischen Diagnostik ist die Rechtwinkel-Parallel-Technik die Technik der Wahl, wobei der Bildempfänger möglichst parallel zum Objekt ausgerichtet wird. Intraoperativ können bei lückenbegrenzenden Einzelzahnimplantaten Zahnaufnahmen mit entsprechenden Markern als Referenz Informationen über die Abstände zu Nachbarzähnen, deren Wurzeln und der Achsstellung in mesiodistaler Richtung geben.

\subsubsection{Transversale Schichtaufnahme}

Durch Zusatzprogramme kann mit entsprechenden Panoramaschichtgeräten eine transversale Schichtaufnahme in einem begrenzten Gebiet erfolgen (FUHRMANN et al. 2003). Dies ermöglicht die Visualisierung einer zusätzlichen Ebene, wodurch die vestibuloorale Breite des Kiefers, die Lage des Canalis mandibulae sowie eine linguale Konkavität des Knochens besser eingeschätzt werden können. Diese Aufnahmetechnik setzt ein besonderes $\mathrm{Maß}$ an Genauigkeit bei der Abstimmung der Projektionsrichtung um den Kieferverlauf voraus. Hierfür müssen spezielle Aufbissbehelfe und Zentriereinrichtungen verwendet werden. Zudem können verfahrensimmanente Unschärfen entstehen, welche die diagnostische Wertigkeit 
der Bilder herabsetzt. Dieses Verfahren kommt heute nur noch sehr selten zum Einsatz.

\subsubsection{Fernröntgen und Aufbissaufnahmen}

Die Fernröntgenaufnahme hat eine eingeschränkte Indikation in der Implantologie, da sie als zusätzliches Diagnoseverfahren nur begrenzte Informationen liefert. Sie dient aber der mandibulo-maxillären Relationsbestimmung in der rekonstruktiv präprothetisch-implantologischen Chirurgie. Zudem kann sie, bei geplanter interforaminaler Implantation, Informationen über die Stärke der Unterkieferspange im Symphysenbereich liefern (WEBER 2010).

Bei einem zahnlosen Patienten kann eine Aufbissaufnahme des Unterkiefers der präoperativen Einschätzung des Knochenangebotes in der Horizontalebene dienen.

\subsubsection{Digitale Volumentomographie}

Die digitale Volumentomographie wurde auf Grundlage der Cone-beam-Technik speziell für die dentale Röntgendiagnostik entwickelt (MOZZO et al. 1998). Sie erlaubt vergleichbare Darstellungen wie bei der Computertomographie, wobei die Hartgewebsdarstellung im Vordergrund steht (KYIAKOU et al. 2011).

Bei der digitalen Volumentomographie läuft eine Röntgenröhre in einer zirkulären Bahn um den Patienten. Dabei wird die Röntgenröhre in der Regel jeweils pro Winkelgrad ausgelöst und die kegelförmig ausgesendeten Röntgenstrahlen (cone beam) mit einem Flachdetektor (flat panel detector, FPD) registriert. Das empfangene Signal wird proportional in Grauwerte umgerechnet. Dabei entsteht ein Schwächungsbild des Messobjektes. Diese ergeben in der Fläche zweidimensionale Summations-Einzelbilder. Der Patient darf sich während der Aufnahmezeit von mehreren Sekunden (je nach Gerät derzeit < 20 s) nicht bewegen.

Mit dem Datensatz können Schnittbilder aller Raumebenen sowie dreidimensionale Ansichten errechnet werden.

Die im Vergleich zum OPG zusätzlichen Informationen in vestibulooraler Richtung ermöglichen eine Beurteilung des Implantatlagers in allen Raumrichtungen (SCHULZE et al. 2013). Zusätzlich können durch eine zweidimensionale Rekon- 
struktion senkrecht zur Horizontalebene Bilder der Zahnreihen erzeugt werden, die einer Panoramaschichtung ähneln. Dabei werden, anders als bei der Panoramaschichttechnik, je nach Auswahl ein einzelnes Schnittbild oder mehrere summierte Schichten hintereinander dargestellt.

Bei der digitalen Volumentomographie sind etwas weniger störende Artefakte durch metallische Restaurationen zu beobachten als bei der Computertomographie. Im Vergleich zu dieser weist die digitale Volumentomographie zudem eine geringere Strahlenbelastung auf (KYRIAKOU et al. 2011; LUDLOW und IVANOVIC 2008). 


\subsection{Strahlenexposition in der Zahnmedizin}

Die jährliche "Ganzkörperbelastung" (effektive Dosis) in Deutschland betrug im Jahr $20114 \mathrm{mSv}$ pro Einwohner, wobei 1,8 mSv auf medizinische Untersuchungen zurückzuführen waren (Bundesamt für Strahlenschutz 2012, Parlamentsbericht). Hierbei machten die CT-Untersuchung und die Arteriographie mit Intervention einen wesentlichen Anteil der kollektiven effektiven Dosis aus.

Während 2011 ca. $39 \%$ der Röntgenaufnahmen in der Zahnmedizin angefertigt wurden, betrug deren effektive Dosis nur 0,3\% der Summe aller medizinischen Untersuchungen (Abb. 1).

Spezielle diagnostische Verfahren in der zahnärztlichen Radiologie wie die Computertomographie oder digitale Volumentomographie führen zu einer deutlich höheren Strahlenexposition bei einzelnen Patienten.

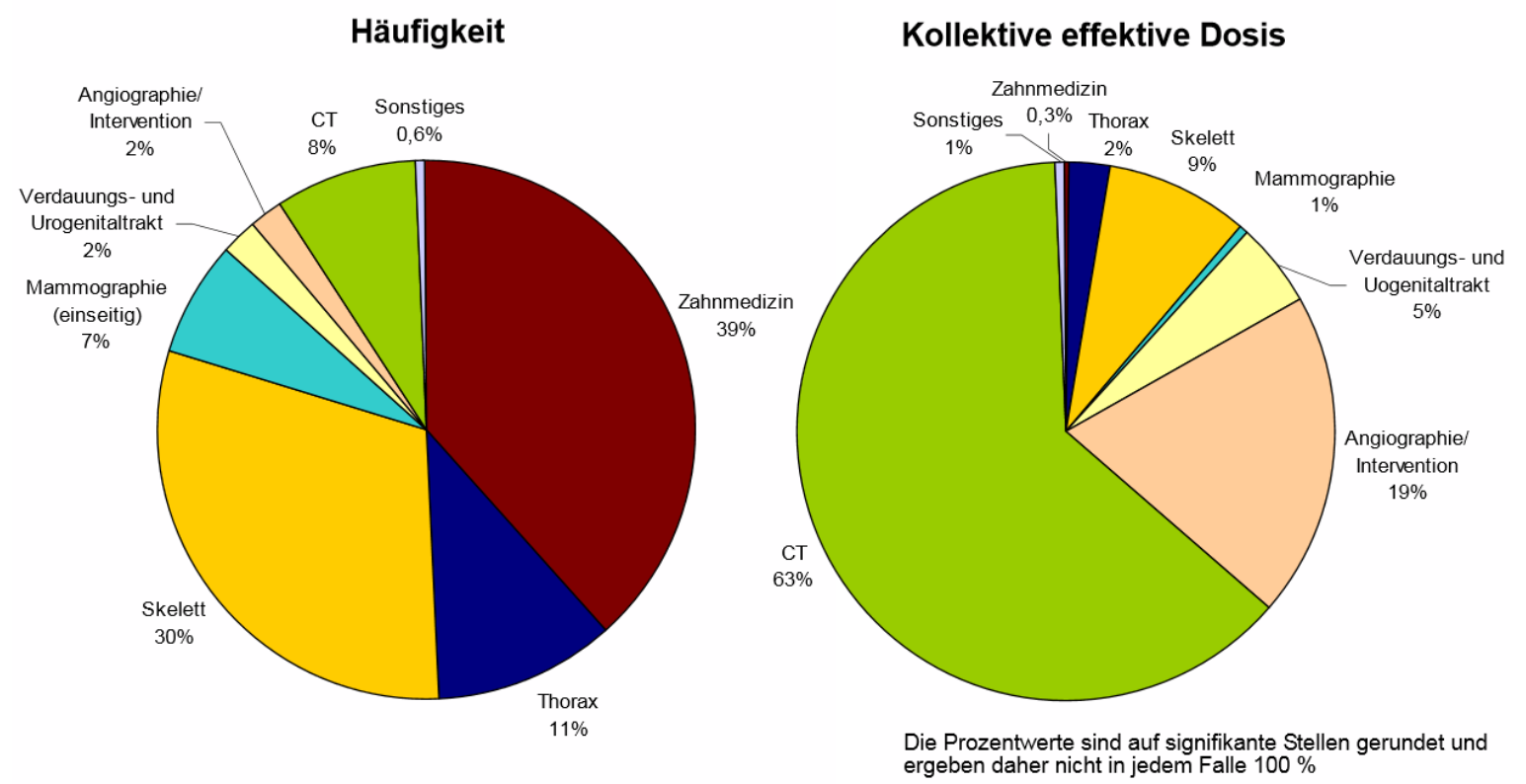

Abb. 1: Prozentualer Anteil der verschiedenen Untersuchungsarten an der Gesamthäufigkeit und an der kollektiven effektiven Dosis für das Jahr 2011 (Abbildung aus Bundesamt für Strahlenschutz 2012, Parlamentsbericht)

Bei den Aufnahmetechniken müssen konventionelle (Film, Verstärkerfolie) von digitalen Verfahren (Sensor, Speicherfolien) unterschieden werden. Letztere ermöglichen eine Dosisreduzierung. 
Eine Panoramaschichtaufnahme führt je nach Gerät und Einstellung zu einer effektiven Dosis in der Größenordnung von 2,7-24,5 $\mu$ Sv. Dagegen führt ein digitales Volumentomogramm zu einer effektiven Dosis von 11-674 $\mu \mathrm{Sv}$. Ein Dental-CT schließlich kann eine effektive Dosis von 180-2100 $\mu$ Sv verursachen. Dies entspricht etwa vier DVT-Aufnahmen (LUDLOW und IVANOVIC 2008). Auch spezielle CT-Protokolle für die Kiefer zeigen eine bis zu siebenfach höhere effektive Dosis bei CT-Aufnahmen im Vergleich zur digitalen Volumentomographie (KYRIAKOU et al. 2011).

Tab. 1: Effektive Dosis konventioneller und dreidimensionaler Röntgenaufnahmen in $\mu$ Sv nach ICRP 2007 (NITSCHE et al. 2012)

\begin{tabular}{|l|l|}
\hline Aufnahmeart & effektive Dosis $(\boldsymbol{\mu S v})$ \\
\hline Fernröntgenseitenaufnahme & 5,6 \\
\hline Panoramaschichtaufnahme (digital) & $2,7-24,5$ \\
\hline Zahnfilmstatus & $34,9-388$ \\
\hline Digitales Volumentomogramm (DVT) & $11-674$ \\
\hline Computertomogramm (CT) & $180-2100$ \\
\hline
\end{tabular}

Eine Metaanalyse von LUDLOW et al. (2015) zur effektiven Dosis von dentalen Volumentomographen zeigt einen sehr breiten Bereich der effektiven Dosen von 5 $\mu \mathrm{Sv}$ bis $1073 \mu \mathrm{Sv}$. Geringere effektive Dosen werden hier meist zulasten des erfassten Volumens oder der Ortsauflösung erreicht. Schutzmaßnahmen wie die Verwendung eines Tyoideaschildes ermöglichen eine Reduktion der effektiven Organdosis von über $40 \%$ bei DVT-Aufnahmen (QU et al. 2012).

KYRIAKOU et al. (2011) zeigen in einer vergleichenden Untersuchung, dass mit dem Mehrschicht-Spiral-CT bei entsprechend an den Gesichtsschädel angepassten Protokollen mit gleicher oder geringerer Strahlenexposition eine bessere Bildhomogenität als beim DVT erreicht werden kann.

Obwohl das Risiko einer Schädigung durch zahnärztliche Röntgenuntersuchungen nach allen vorliegenden Daten außerordentlich klein ist, sollte man bestrebt sein, die Strahlenexposition für den Patienten möglichst gering zu halten (Bundesamt für Strahlenschutz 2003, § 2c Röntgenverordnung). Im Bereich der präimplantologischen Röntgendiagnostik kann die Verbesserung der Aussagekraft von Verfahren mit relativ geringer Stahlenexposition dazu einen Beitrag leisten. 


\subsection{Zielsetzung}

Ziel der Arbeit war der Vergleich verschiedener Röntgenmarkersysteme zur präoperativen Planung vor einer Implantatinsertion mit der Panoramaschichtaufnahme. Dazu sollte die metrische Genauigkeit von vier verschiedenen gängigen Röntgenmarkern miteinander verglichen werden. Zusätzlich sollten die Lage der Röntgenmarker zur crestalen Knochenkante und deren Kippung in vestibulooraler Richtung differenziert betrachtet werden.

Für jeden Markertyp wurde unter gleichen Bedingungen eine Planungsschablone angefertigt, die anschließend zusammen mit einem weiterentwickelten Röntgenmodell eines Unterkiefers in einem digitalen Panoramaschichtgerät geröntgt wurde. Die dabei entstehenden Orthopantomogramme wurden von Versuchsteilnehmern vermessen.

Zudem wurden Aufnahmen des Röntgenmodells mit einer Planungsschablone mit einem digitalen Volumentomographen erstellt und von Versuchsteilnehmern vermessen. Die Ergebnisse wurden denen aus der Panoramaschichttechnik gegenübergestellt.

Insbesondere folgende Fragestellungen sollten bearbeitet werden:

- Gibt es Unterschiede in der Genauigkeit der Bestimmung des vertikalen Knochenangebotes bei der Verwendung verschiedener Röntgenmarkersysteme und welches liefert die genauesten Ergebnisse?

- Gibt es Unterschiede in der Auswertung der Röntgenaufnahmen, die vom Untersuchungsgebiet abhängig sind?

- Liefert der Einsatz einer digitalen Schieblehre im Vergleich zu einem handelsüblichen Geometriedreieck bei der Vermessung von ausgedruckten Orthopantomogrammen genauere Messwerte?

- Kann in einer Aufnahme mit einem digitalen Volumentomographen (DVT) unter Idealbedingungen eine höhere metrische Genauigkeit bei der Vermessung des vertikalen Knochenangebotes erreicht werden als bei der Panoramaschichttechnik unter Zuhilfenahme einer Markerschablone? 


\section{Material und Methoden}

\subsection{Erstellen eines Röntgenmodells}

\subsubsection{Prothetische Überlegungen}

Für die Untersuchungen wurde als typischer Fall die Kennedyklasse II mit einer unilateralen Freiendsituation im Unterkiefer gewählt. Eine mögliche prothetische Lösung für diesen Fall wäre ein herausnehmbarer Zahnersatz in Form einer Modellguss- oder Teleskopprothese. Bei dieser Art von Versorgung ist eine zusätzliche Abstützung auf der Gegenseite unter Einsatz eines Sublingualbügels erforderlich. Zur Verankerung des Zahnersatzes müssten hierbei gegebenenfalls Zähne beschliffen werden. In beiden Fällen wäre eine gemischt parodontal-gingivale Lagerung des Zahnersatzes unumgänglich, die vor allem bei einem bezahnten antagonistischen Kieferabschnitt zu Problemen führen kann wie Schleimhautirritationen oder verstärkter parodontaler Belastung und einem erhöhten Frakturrisiko an den lastnahen Zähnen (STRUB et al. 2005, S. 901). In jedem Fall entstehen durch eine Lage der Prothesenzähne außerhalb des Unterstützungspolygons ungünstige Hebelwirkungen.

Eine klinisch sinnvolle Alternative ist die Insertion von Implantaten im zahnlosen Kieferabschnitt zur Pfeilervermehrung. Diese dienen je nach Planung der Aufnahme von Einzelzahnkronen oder einer Brücke (RICHTER 2005; AUGTHUN und MUNT 2008). Bei vollständiger Gegenbezahnung wären bei der Planung mindestens zwei Implantate nötig.

\subsubsection{Das Röntgenmodell}

Für die geplanten Messaufnahmen wurde ein Röntgenmodell erstellt. Dadurch konnte mit verschiedenen Schablonen beliebig oft geröntgt werden. Hierzu wurde zunächst ein Unterkiefermodell zu einem Röntgenmodell weiterentwickelt, wofür mehrere Testläufe notwendig waren. Ziel war ein standardisiertes Modell, mit dessen Hilfe die zu untersuchenden Parameter erfasst werden konnten. Dazu mussten alle weiteren Einflussfaktoren möglichst ausgeschlossen werden. 
Als Basis diente ein Unterkiefermodell aus röntgenopakem Kunststoff, das für Implantatfortbildungen entwickelt und verwendet wurde (zur Verfügung gestellt von Dr. Bohne/Northeim). Das Röntgenmodell wurde aus einem autopolymerisierenden Kunststoff gefertigt, dessen Röntgenopazität durch Bariumsulfat erhöht wurde.

Das Modell bestand aus dem Unterkieferkorpus mit angedeuteten aufsteigenden Ästen. Im Alveolarfortsatz wies es Nachbildungen der klinischen Kronen von 37 bis 47 mit fehlenden Zähnen 35, 31, 41 und 45 auf. Mehrere Modifikationen mussten an diesem Modell durchgeführt werden, um den Ansprüchen der geplanten Versuchsreihen zu genügen.

\subsubsection{Modifikationen des Grundmodells}

Die ersten Probeaufnahmen des Grundmodells des Unterkiefers erfolgten mit Hilfe des digitalen Panoramaschichtgerätes Orthophos XG Plus (Siemens) in der Röntgenabteilung des Zentrums für Zahn-, Mund- und Kieferheilkunde der Universitätsklinik Göttingen. Dabei wurde ein Kamerastativ (Vanguard CT-2888) mit Plexiglasaufbau zur Ausrichtung des Unterkieferkorpus verwendet.

Eine Ausrichtung nach der Frankfurter Horizontalen ausschließlich mit dem Unterkiefermodell war nicht möglich, da sich die entsprechenden anatomischen Strukturen am Viszerokranium befinden und somit am Modell nicht verfügbar waren.

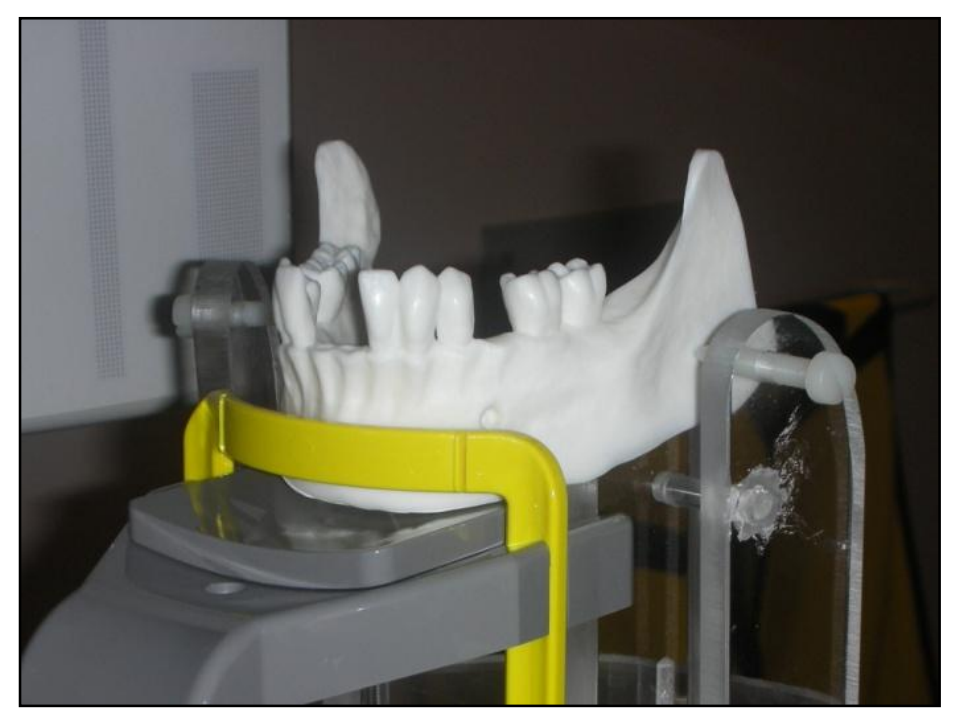

Abb. 2: Ursprüngliches Röntgenmodell fixiert auf Fotostativ im digitalen Panoramaschichtgerät Orthophos XG Plus 
Somit war die Bestimmung der Okklusionsebene allein anhand des Unterkiefermodells notwendig. Um den Interinzisalpunkt zu definieren wurden von einem zweiten Modell die Zähne 32 und 42 mit einer Laubsäge getrennt, seitlich verschmälert und mit Kronenwachs am Modell in Position 31 und 41 fixiert.

Danach wurden die beiden Zähne 46 und 47 mit einem Sägeschnitt auf Höhe der crestalen Knochenkante horizontal vom Modell gesägt. Mit einem Technikerhandstück und einer feinverzahnten Fräse wurde im Bereich der für den Versuch relevanten Regio 46/ 47 die Form einer eindeutigen crestalen Knochenkante ausgearbeitet. Dieses Modell wurde mit einer Beimengung von $\mathrm{BaSO}_{4}$ in einer Konzentration von 0,3 und $0,6 \%$ dupliziert.

Um die Messung der vorhandenen realen Knochenhöhe zu ermöglichen, wurde im Modell ein Nervkanal im Sinne des Canalis mandibulae angelegt. Des Weiteren mussten die Implantationsregion und Implantationsrichtung definiert werden. So sollte sichergestellt werden, dass die Versuchsteilnehmer bei der späteren Auswertung der Panoramaschichtaufnahmen dieselbe Implantatregion und Implantatausrichtung vermessen konnten.

Als erstes wurde die Okklusionsebene beidseitig auf den aufsteigenden Ästen des Unterkiefermodells mit einem dünnen wasserfesten Stift markiert. Als Hilfsmittel diente hierbei eine plane Wachsplatte. Diese reichte in ihrer viertelkreisförmigen Ausdehnung mit der Spitze bis zum Inzisalpunkt und bis zu den distobukkalen Höckern der zweiten unteren Molaren und entsprach somit der Okklusionsebene im Sinne eines erweiterten Bonwilldreiecks (LEHMANN und HELLWIG 2012, S. 64).

Anschließend wurde das Modell entlang des geplanten Verlaufes des Nervkanals mit Hilfe einer diamantierten Bandsäge (Exakt Trennschleifsystem Standard) mit einer Schnittbreite von 0,1 mm gesägt. Der Schnitt lag senkrecht zur Okklusionsebene, entlang der crestalen Knochenkante. 


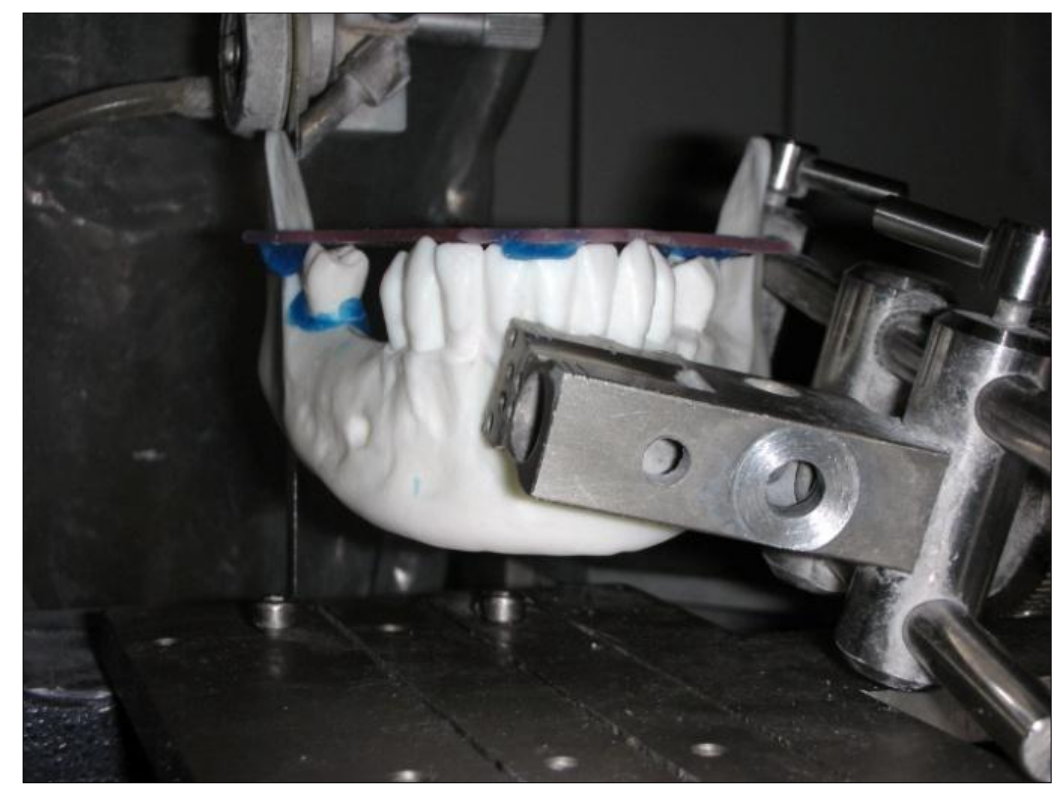

Abb. 3: Ausrichten des Unterkiefermodelles an der Bandsäge senkrecht zur Okklusionsebene

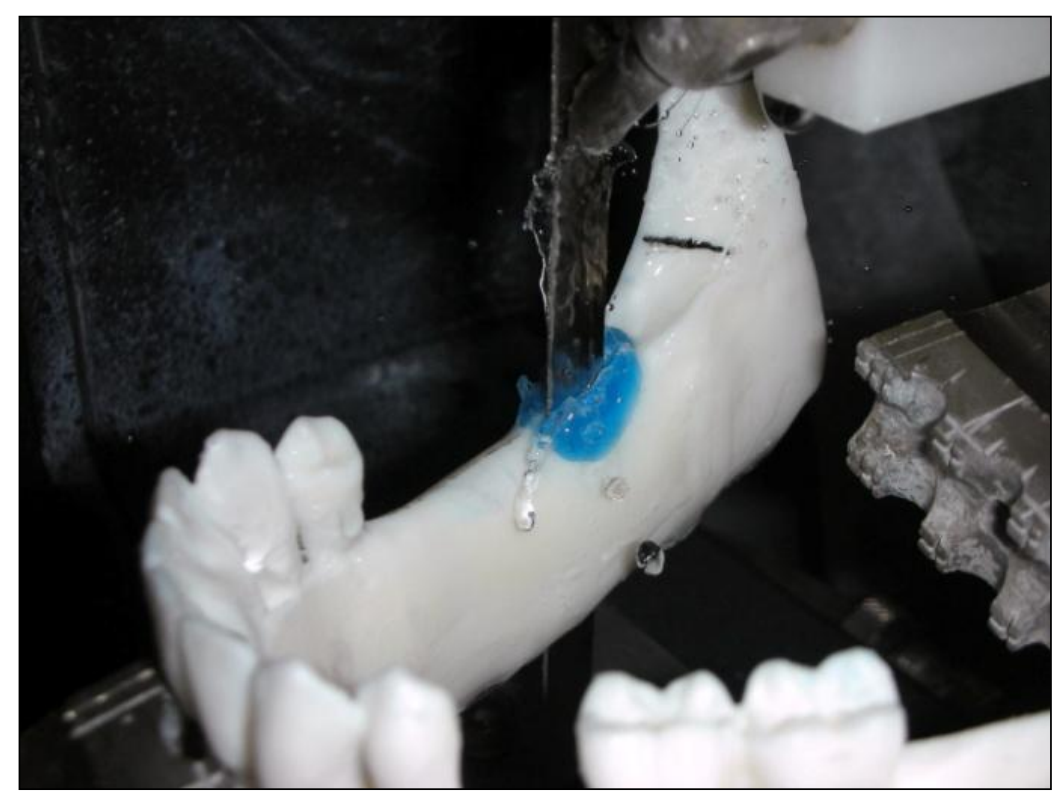

Abb. 4: Sägen des Unterkiefermodells mit einer diamantierten Bandsäge crestal und senkrecht zur Okklusionsebene

Zur exakten Ausrichtung des Modells in der Bandsäge wurden Wasserwaagen, eine senkrecht zur Okklusionsebene liegende Wachsplatte (Beauty Pink) und ein Lot verwendet. Die Fixierung des Unterkiefermodells erfolgte über eine fest mit der Sägevorrichtung verbundenen Schraubvorrichtung. Unter Wasserkühlung wurde dann das Modell von distal nach mesial bis kurz anterior vor das Foramen mentale gesägt. Anschließend erfolgte ein Schnitt von lateral distal des Zahnes 44, um das Modell zu teilen. 
Zur Darstellung des Verlaufs des Canalis mandibulae wurde mit dem Technikerhandstück und einer Kugelfräse (Durchmesser: 2,75 mm) ein Nervkanal jeweils zur Hälfte proximal und lateral in die beiden Modellteile gefräst. Um den Nervkanal auch röntgenologisch darstellen zu können, diente eine $40 \mathrm{~mm}$ lange und 2,5 mm dicke Kabelhülle als röntgenopake, kortikale Kanalwand. Zur Befestigung und zum Ausgleich des geringen Volumendefizites wurde Zahnwachs benutzt. Der Volumenverlust zwischen den beiden Modellteilen, den das Sägen verursachte, wurde ebenfalls mit einer $0,1 \mathrm{~mm}$ dünnen, gleichmäßigen Wachsschicht ausgeglichen.

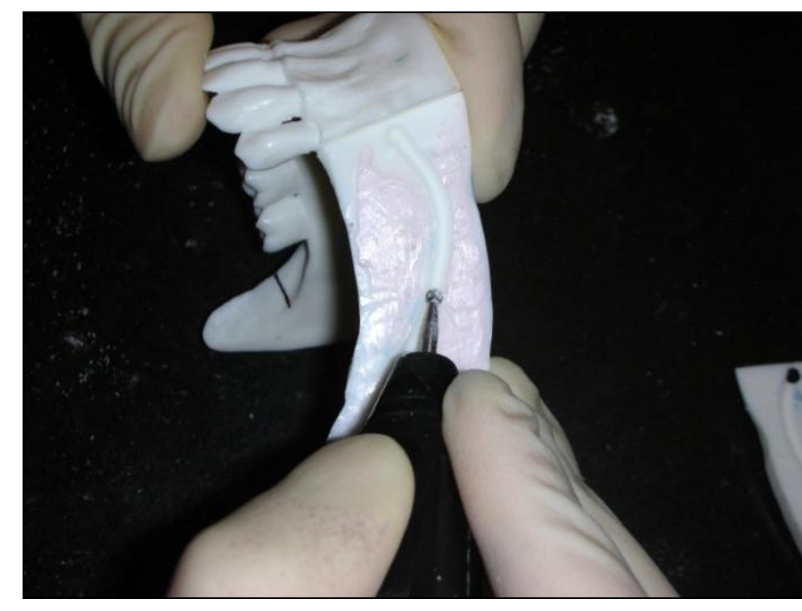

Abb. 5: Fräsen des Kanals

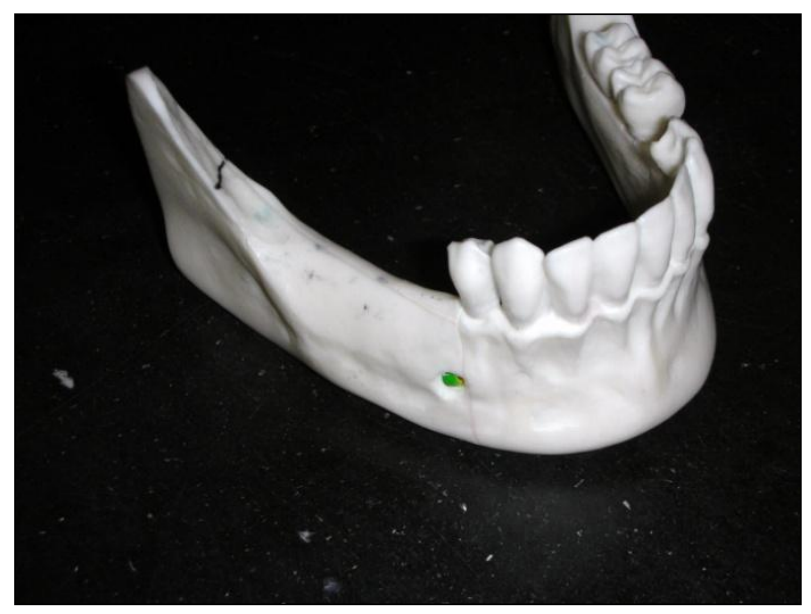

Abb. 6: Fertiges Röntgenmodell

Als genau definierbare Messpunkte (Referenzpunkte) für die Knochendicke über dem Nervkanal wurden zusätzlich an den beiden geplanten Implantationsorten jeweils crestal und direkt oberhalb des Nervkanals halbkugelförmige Aussparungen gefräst. Diese wurden mit röntgenopaker Guttapercha (Guttaperchapoints) gefüllt. Dieser Vorgang erfolgte durch Schmelzen der Guttapercha mit Hilfe eines heißen Wachsmessers und Modellieren mit einem LeCron-Instrument. Mit einem Skalpell konnten die Überschüsse sauber entfernt werden.

Die beiden Messpunkte für Regio 46 wurden auf die linguale Modellhälfte gesetzt und die für Regio 47 auf das vestibuläre Teilstück des Unterkiefermodells. Somit lagen die Messpunkte aus Guttapercha nach Ausrichten im Röntgengerät genau übereinander in der Transversalen senkrecht zur Okklusionsebene und konnten auch beim Zusammensetzen des Modells ihren Abstand zueinander nicht verändern. 
Diese Referenzpunkte aus Guttapercha sollten die Implantationsstellen und die Richtung der geplanten Implantation definieren, um die späteren Messungen immer an derselben Stelle durchführen zu können.

\subsubsection{Röntgenschablonen und Markersysteme}

Vor einer Implantat-OP wird eine Situationsabformung des entsprechenden Kiefers vorgenommen. Diese wird ausgegossen. Auf dem entstehenden Gipsmodell wird eine Tiefziehschablone angefertigt. Diese Schablone erfültt mehrere Aufgaben. Zum einen dient sie präoperativ der Abschätzung des vorhandenen Knochenangebotes, zudem kann sie intraoperativ als Positionierungs- und Richtungshilfe für die Pilotbohrung genutzt werden (GUTWALD et al. 2010). Diese Vorgehensweise wurde für die Versuche nachvollzogen.

Verschiedene Markersysteme, die sich in Material, Länge und geometrischer Form unterscheiden, können dafür auf der Tiefziehschablone angebracht werden. Für den Versuch sollte die Genauigkeit von folgenden Markersystemen miteinander verglichen werden:

- Titanhülsen aus Reintitan,

- Kugellagerkugeln,

- Titanplättchen und

- Guttaperchapoints mit individuellen Längen. 
Tab. 2: Verwendete Markersysteme

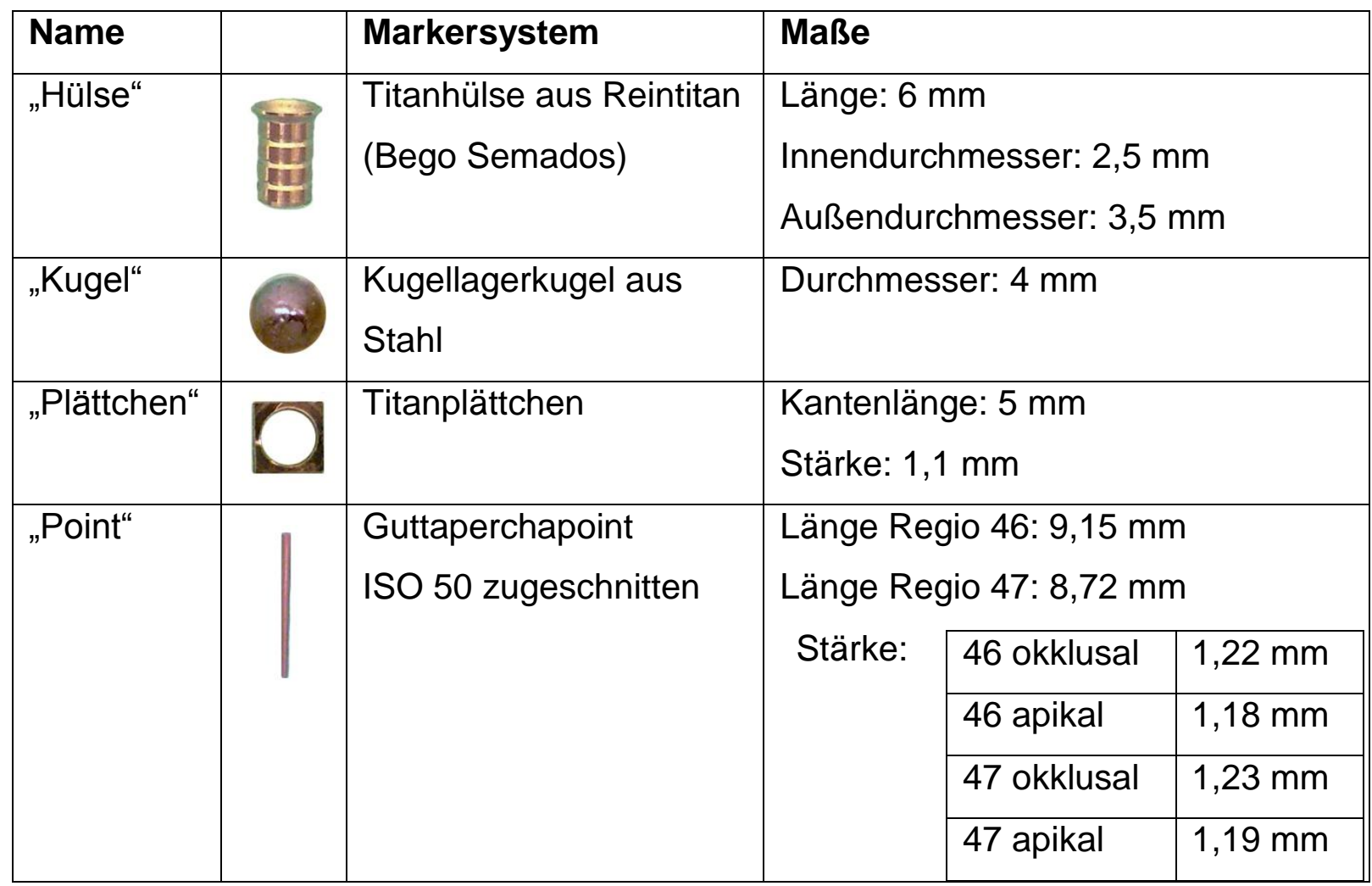

Für jeden Röntgenmarkertyp wird eine eigene Kunststoff-Röntgenschablone hergestellt. Die für die Versuchsreihe geplanten Positionen der Marker auf den entsprechenden Röntgenschablonen sind:

Standardisierte Bohrhülsen, welche in der Referenzierungsschablone crestal der Implatationsregion platziert werden.

Standardisierte Kugeln, welche in der Referenzierungsschablone crestal der Implatationsregion platziert werden.

Standardisierte Plättchen, welche in der Referenzierungsschablone bukkal der Implatationsregion platziert werden.

Guttaperchapoints, welche in der Referenzierungsschablone lingual der Implatationsregion platziert werden.

Die metrischen Angaben zur Lage der unterschiedlichen Marker relativ zur Kieferform sind in Tab. 3 zusammengefasst. 
Tab. 3: Horizontaler Abstand der Marker zur crestalen Knochenkante

\begin{tabular}{|l|l|l|l|l|}
\hline Regio & \multirow{2}{*}{$\begin{array}{l}\text { Hülse } \\
\text { (crestal) }\end{array}$} & $\begin{array}{l}\text { Kugel } \\
\text { (crestal) }\end{array}$ & $\begin{array}{l}\text { Plättchen } \\
\text { (vestiulär) }\end{array}$ & $\begin{array}{l}\text { Point } \\
\text { (lingual) }\end{array}$ \\
\cline { 1 - 3 } 46 okklusal & & & $6,33 \mathrm{~mm}$ & $3,20 \mathrm{~mm}$ \\
\cline { 1 - 1 } 46 apikal & \multirow{3}{*}{$0 \mathrm{~mm}$} & \multirow{2}{*}{$0 \mathrm{~mm}$} & $6,33 \mathrm{~mm}$ & $3,20 \mathrm{~mm}$ \\
\cline { 1 - 1 } 47 okklusal & & $5,72 \mathrm{~mm}$ & $2,15 \mathrm{~mm}$ \\
\cline { 1 - 1 } 47 apikal & & $6,25 \mathrm{~mm}$ & $2,75 \mathrm{~mm}$ \\
\hline
\end{tabular}

Der Marker in Regio 47 wurde jeweils um $10^{\circ}$ nach lingual gekippt aufgestellt. Dies schließt die Überlegung ein, dass bei der zahntechnischen Produktion der Schiene nicht in jedem Fall eine Ausrichtung der Röntgenmarker mit Hilfe eines Parallelometers erfolgt. Zudem werden Marker, die auch der Führung des Pilotbohrers dienen sollen (Bohrhülse), nach prothetischen Aspekten ausgerichtet. So können im Unterkieferseitenzahnbereich die Marker nach lingual gekippt aufgestellt werden, um die natürliche Lingualkippung des anterioren Restgebisses und den anatomischen Aufbau des Unterkiefercorpus in der Transversalen zu berücksichtigen.

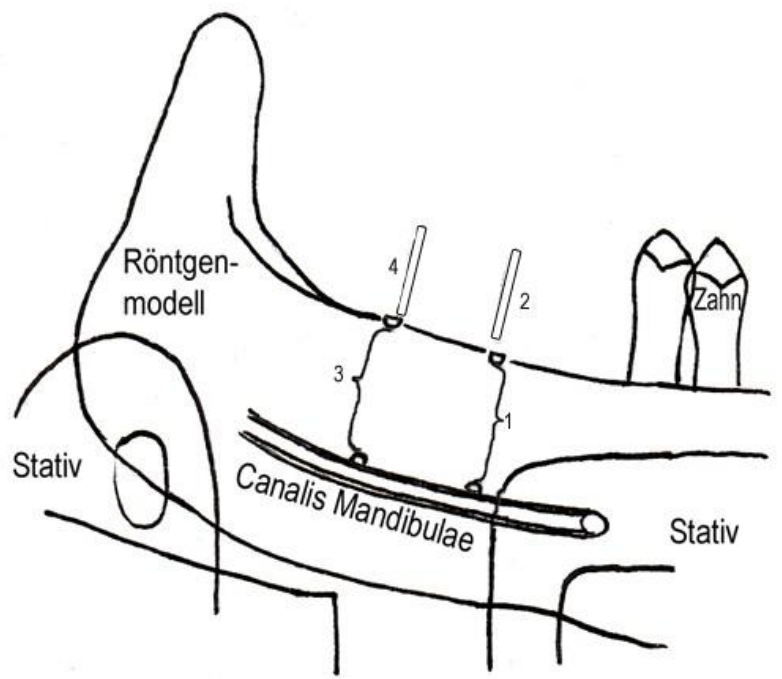

Abb. 7: Planzeichnung des Unterkiefermodels mit den zu vermessenden Strecken (1-4)

1 Distanz für die Referenzierungsmarker in Regio 46

2 Röntgenmarker für Regio 46

3 Distanz für die Referenzierungsmarker in Regio 47

4 Röntgenmarker für Regio 47 


\subsubsection{Geometrische Überlegungen zu den verwendeten Markersystemen}

In Zentralstrahlrichtung sehr schmale Objekte, die gekippt zum Zentralstrahl geröntgt werden, erscheinen verkürzt auf dem Röntgenbild. Die verwendeten Röntgenmarker sind dreidimensionale geometrische Formen. Daher ist es möglich, dass bei einer mäßigen Kippung des Markers zur Senkrechten des Zentralstrahls seine Projektion auf die zweidimensionale Röntgenebene diese Kippung kompensiert oder sogar zu einer längeren Abbildung führt.

Die Projektion der Titanhülse lässt sich vollständig mit zwei Dimensionen erklären. Hier wird aus dem Querschnitt eines Zylinder bzw. stumpfen Kegels ein Rechteck bzw. ein Trapez.

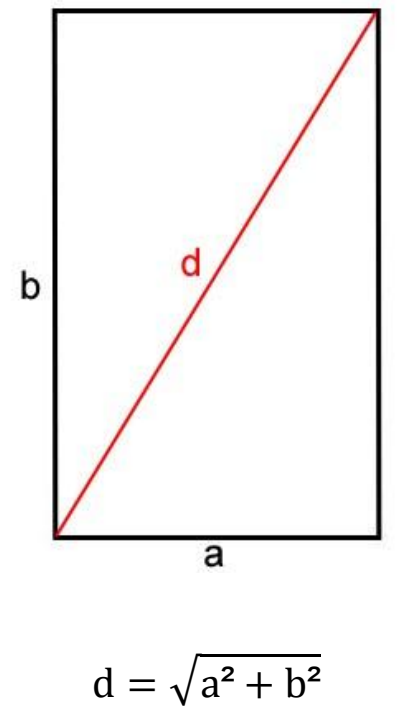

Abb. 8: Diagonalenlänge

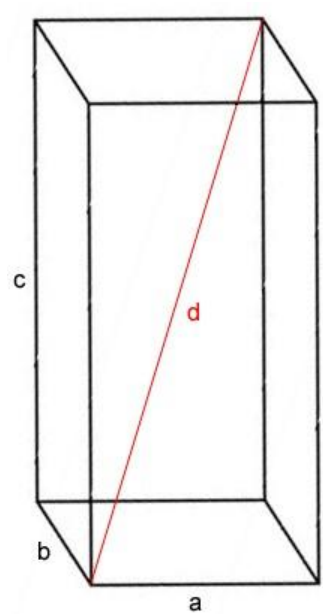

$$
d=\sqrt{a^{2}+b^{2}+c^{2}}
$$

Abb. 9: Länge der Raumdiagonalen

Die Kugel ist in der Anordnung vollkommen unproblematisch, da sie radialsymmetrisch ist. Nur hier können Verzeichnungen ausschließlich durch das bildgebende System entstehen. Die Kugel muss bei der Planung der Röntgenschablonen möglichst crestal über dem Implantatlager angebracht werden, um zu gewährleisten, dass sie bei der Panoramaschichtaufnahme in derselben Schicht wie das Knochenlager liegt.

Da das Titanplättchen eine gewisse Stärke aufweist, trifft hier eine dreidimensionale Betrachtungsweise zu. Ein dreidimensionaler Körper, z.B. ein Quader mit quadratischer Grundfläche, hat seine maximale Länge im Raum und nicht an seiner 
längsten Kante. Die längste Ausdehnung des Quaders und damit auch mögliche Projektion ist seine Raumdiagonale. Die Projektion des dünnen Guttaperchapoints lässt sich wieder vollständig mit zwei Dimensionen erklären.

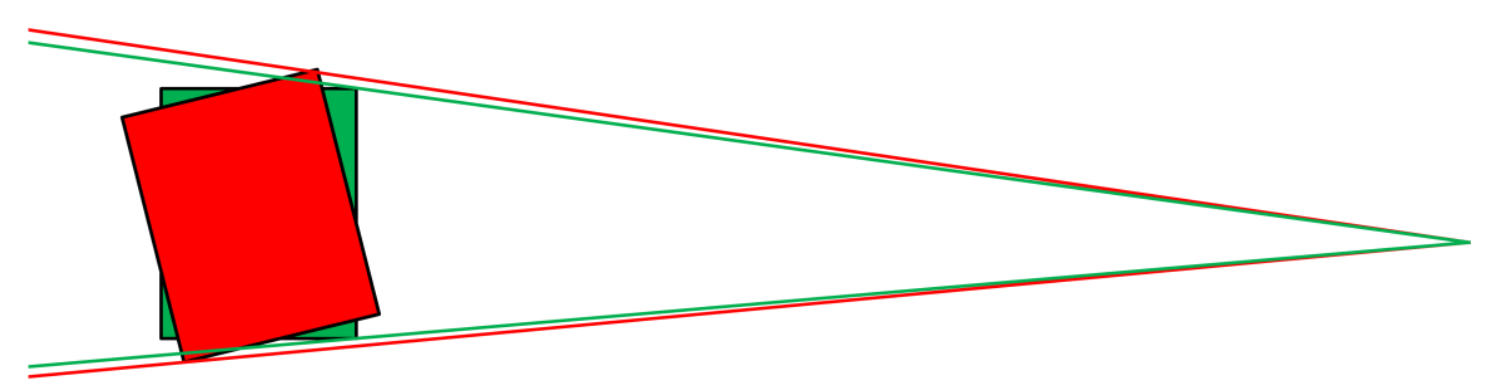

Abb. 10: Dicker Marker wie der Hülse mit Kippung in vestibulooraler Richtung

Es muss beachtet werden, dass im Strahlengang tiefe Körper, die leicht gekippt zur Senkrechten des Zentralstrahls stehen, ein etwas größeres Abbild auf einem Röntgenfilm bzw. Sensor hinterlassen als ein in Richtung des Strahlengangs schmales Objekt. Dieses erscheint in seiner Abbildung, je nach Kippungsgrad, weniger hoch als es in Wirklichkeit ist.

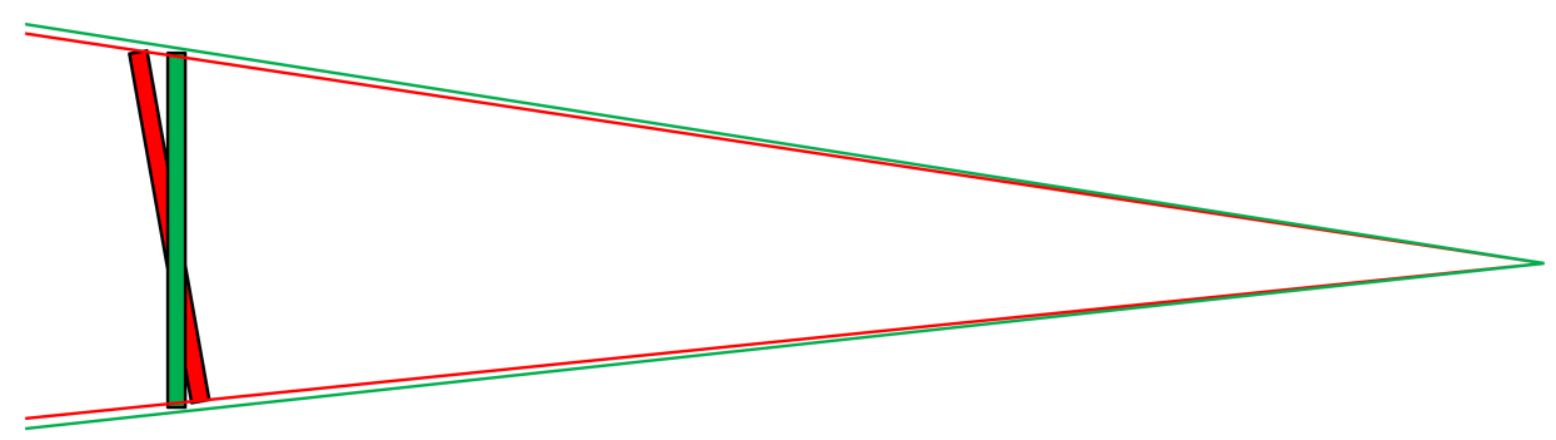

Abb. 11: Schmaler Marker wie der Guttaperchapoint mit Kippung in vestibulooraler Richtung

Weiterhin muss bedacht werden, dass die Stellung der Marker in bukkulingualer Richtung in der Röntgenschicht zusammen mit der Kippung zur Senkrechten des Zentralstahls dazu führen kann, dass sich vergrößernde und verkleinernde Effekte in der Projektion aufheben oder verstärken können. 


\subsubsection{Herstellung der Röntgenschablonen}

Um die Kunststoffschablonen herzustellen, auf denen die verschiedenen Röntgenmarker fixiert werden sollten, wurde das Modell abgeformt. Dazu wurde ein Abformlöffel (AlgiLock BI-3) mit Kerr angepasst, die Zahnzwischenräume und das Foramen mentale mit Peripheriewachs ausgeblockt und die Abformung mit Alginat vorgenommen. Nach Ausgießen mit Superhartgips wurde das Gipsmodell auf der Unterseite senkrecht zur Okklusionsebene plan getrimmt (Wassermann HSS88). Als Hilfsmittel dienten eine Kunststoffplatte, welche mit Stopps aus Palavit G und Peripheriewachs an dem Unterkiefermodell parallel zur Okklusionsebene ausgerichtet wurde, und eine Wasserwaage. Des Weiteren wurde das Gipsmodell zirkulär bis zur Umschlagfalte und distal bis zu den aufsteigenden Ästen des Unterkiefers zurückgetrimmt und gefräst. Mit Hilfe dieses Modells wurde eine Silikon-Negativform erstellt. Dazu wurde das Modell in eine Dublierform (Wiroil (Bego) gestellt und diese mit flüssigem Silikon (Adisil Rapid (Siladent) aufgefüllt. Nach dem Abbinden des Silikons wurden mit der erzeugten Form vier identische Superhartgipsmodelle hergestellt. Zur Oberflächenentspannung der Silikonform diente dabei KKD Realase Spray. Ein ebener Boden der Modelle wurde durch Auflegen einer kleinen dicken Glasplatte auf die über den Rand mit Superhartgips gefüllte Silikonform erreicht. So konnten mit leichten, gleichmäßigen Schlägen unter Ausnutzung der tixotrophen Eigenschaften des Gipses die Überschüsse verdrängt werden. An den so hergestellten Superhartgipsmodellen konnten die Grenzen zu den unter sich gehenden Stellen mit Hilfe einer in ein Parallelometer eingespannten Graphitmine markiert werden. Caudal dieser Regionen wurde das Gipsmodell großzügig mit Silaplast (Futura + Katalysator) ausgeblockt.

Anschließend erfolgte das Tiefziehen der Schiene mit Hilfe eines Vakuumtiefziehgerätes (Biostar). Für die Tiefziehschablonen wurden 2 mm dicke Kunststoffplatten (Erkodent Erkodur) verwendet. In das Tiefziehgerät eingespannt, wurden diese auf $220^{\circ} \mathrm{C}$ erhitzt (Einstellungscode: 192). Der Aufheizvorgang war nach 65 Sekunden abgeschlossen und die Folie wurde auf das ausgeblockte Modell gestürzt und durch das Vakuum an die Modelloberfläche gezogen. Nach einer Abkühlphase von 60 Sekunden konnte das Gerät entlüftet und das Modell samt Tiefziehfolie entfernt werden. 
Die Ausdehnung der Schablone wurde mit einem wasserfesten Stift auf der Tiefziehfolie festgelegt. Anschließend konnten mit einem Lötkolben (Engel-Löter 100S) die Überschüsse entfernt werden. Die Ränder wurden daraufhin mit einer feinen Fräse ausgearbeitet und mit feinem Bimsstein geglättet.

\subsubsection{Anbringen der Röntgenmarker}

Die Schablone für die Hülsenmarker musste vor dem Anbringen der Marker wie folgt bearbeitet werden. In Regio 45 bis 47 wurden die fehlenden Zähne in ihrer späteren prothetischen und implantologisch sinnvollen Stellung auf dem Kieferkamm aufgewachst (Modellierwachs Rosa Pluradent). Über diese Zähne erfolgte eine Silaplastabformung, die anschließend zusammen mit der Tiefziehschablone als Hohlform diente. In diesen wurde ein Kaltpolymerisat (Weitur Press) eingebracht. Dazu mussten die Wachszähne vorher abgeschmolzen und von distal ein Loch in die Silaplastabformung gebohrt werden. Zum Aushärten des eingegossenen Kaltpolymerisats musste die Schablone für 20 Minuten in einen Drucktopf (2,5 bar) gegeben werden. Für die Titanhülsen wurden von okklusal jeweils durch den Schablonenzahn 46 und 47 ausreichend dimensionierte Löcher gebohrt. Um diese senkrecht zur Okklusionsebene auszurichten, wurde ein Parallelfräsgerät (Fräsgerät F1 Degussa) eingesetzt. Dabei wurden, beginnend mit einem Rosenbohrer (4,5 mm) für eine Pilotbohrung, Fräsen (Spiralbohrer aus Hochleistungsschnellstahl) mit immer größerem Durchmesser verwendet. Die Hülsen wurden mit Wachs in der Schablone fixiert.

Zur Fixierung der Kugellagerkugel wurde auf eine der Schablonen in Regio 46 und 47 jeweils mit einem Rosenbohrer (Durchmesser: $4 \mathrm{~mm}$ ) eine Kuhle bis auf die crestale Kante des Gipsmodells gebohrt und anschließend jeweils eine Kugellagerkugel mit Sekundenkleber geklebt. Die Kugeln wurden mit einer dünnen Schicht Kaltpolymerisat überzogen, um sie sicher zu fixieren.

Auf einer weiteren Schablone, auf der die fehlenden Zähne durch Kaltpolymerisat ergänzt wurden, wurden zwei Metallplättchen jeweils vestibulär der Schablonenzähne 46 und 47 befestigt und mit Wachs fixiert.

Für die Guttaperchapoints musste zunächst wie für die Titanhülsen mit dem Parallelfräsgerät ein Loch in die durch Kaltpolymerisat ergänzten Zähne gebohrt 
werden. Zusätzlich wurde die vestibuläre Wand der aufgestellten Kunststoffzähne 46 und 47 entfernt. Die Guttaperchapoints wurden an der lingualen Wand des Fräsloches mit Wachs fixiert.

\subsubsection{Einstellen der Marker}

Die Ausrichtung der Marker erfolgte mit einem einstellbaren Konuswinkel, der in das Parallelfräsgerät eingespannt wurde. Dabei wurden jeweils in Regio 47 die Hülse, das Plättchen und der Guttaperchapoint um 10ํㅡ nach lingual gekippt aufgestellt.
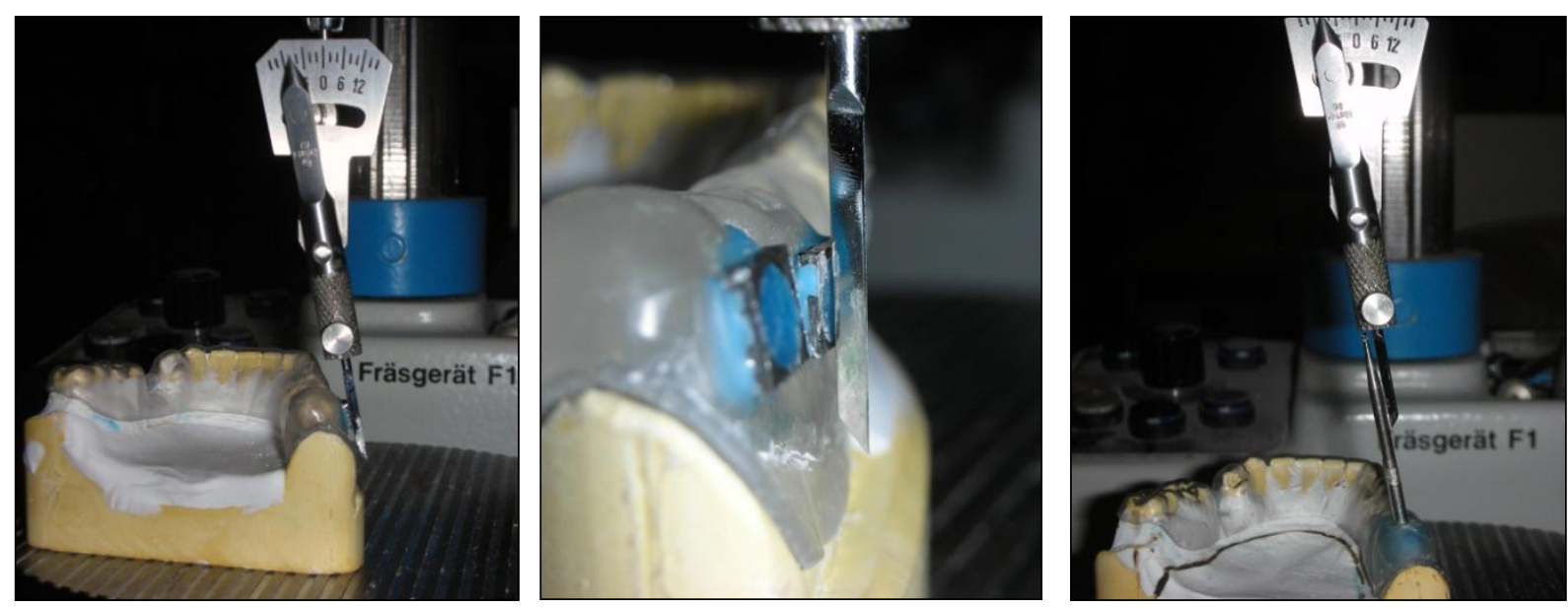

Abb. 12: Ausrichten der Röntgenmarker mit Hilfe eines Parallelfräsgerätes und eines Konuswinkelmessers

\subsubsection{Erstellen der Panoramaschichtaufnahmen}

Für die Versuchsreihe wurde das digitale Panoramaschichtgerät Orthophos XG Plus in der zahnärztlichen Röntgenabteilung des Zentrums für Zahn-, Mund- und Kieferheilkunde des Universitätsklinikums Göttingen verwendet. Mit dem verwendeten Röntgengerät war es möglich, mit geringen $\mathrm{kV} / \mathrm{mA}$-Werten $(60 \mathrm{kV}$ und $3 \mathrm{~mA}$ ) zu röntgen. Da zwischen der konventionellen und dem digitalen Aufnahmeverfahren im Ergebnis des Röntgenbildes kein Unterschied besteht (PASLER 2008) und dieses Verfahren auch in der Praxis immer breitere Anwendung findet, wurde diese Technik gewählt.

Das Röntgenmodell wurde parallel zur Okklusionsebene (PASLER und VISSER 2000 und PASLER und VISSER 2003) und der Mittellinie in das digitale Panorama- 
schichtgerät (Sirona Orthophos XG Plus) eingebracht. Aufgrund der fehlenden Bezugspunkte am Neurocranium und der individuellen Schwankungen der Frankfurter Horizontalen (vergleiche 2.1.3) wurde für die durchgeführten Versuche das Modell senkrecht zur definierten Okklusionsebene, parallel zur vorgegebenen Implantationsrichtung und den platzierten Markern ausgerichtet. Ein Kamerastativ (Vanguard CT-2888) und die Kinnstütze des Panoramaschichtgerätes dienten dabei dem Halt des Unterkiefermodells. So konnte es sicher fixiert werden, was das Wechseln der verschiedenen Markerschienen zwischen den Aufnahmen erleichterte. Anschließend wurden mit den verschiedenen Schablonen je eine Orthopantomogrammaufnahme mit den Einstellungen $60 \mathrm{kV}$ und $3 \mathrm{~mA}$ angefertigt und digital gespeichert. Für die Aufnahmen wurde das Programm P1 $\mathrm{C}$ aus der Programmgruppe Panorama Aufnahmen gewählt. Dieses ermöglicht Normalaufnahmen mit einer konstanten 1,25-fachen Vergrößerung und wird vom Hersteller Siemens für die Verwendung in der Implantologie empfohlen. Die vier Aufnahmen wurden in befundtauglicher Qualität auf Thermoprintfolien im Maßstab 1:1 ausgedruckt (Codonics np-1660m medical print). 


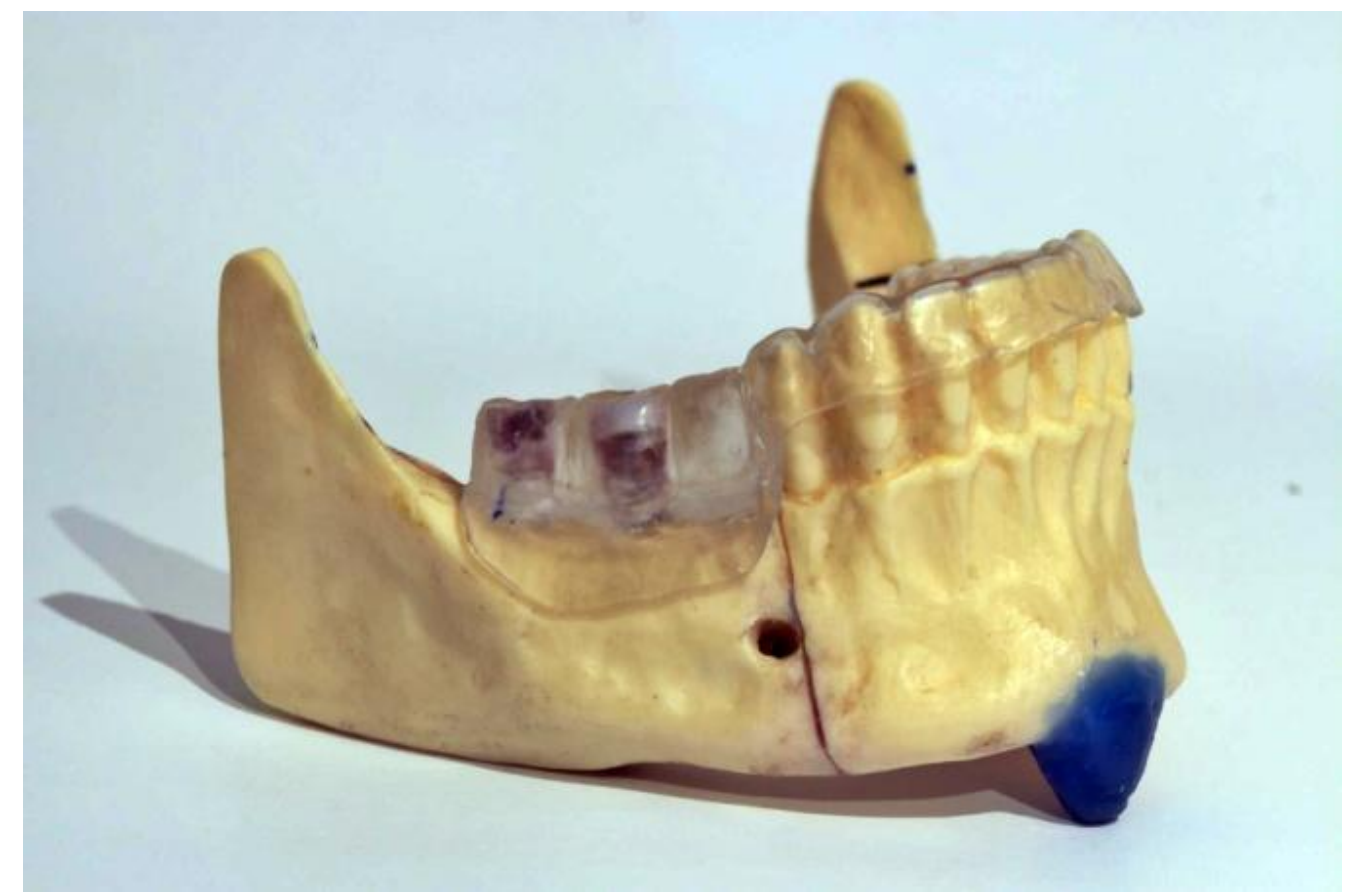

(a)

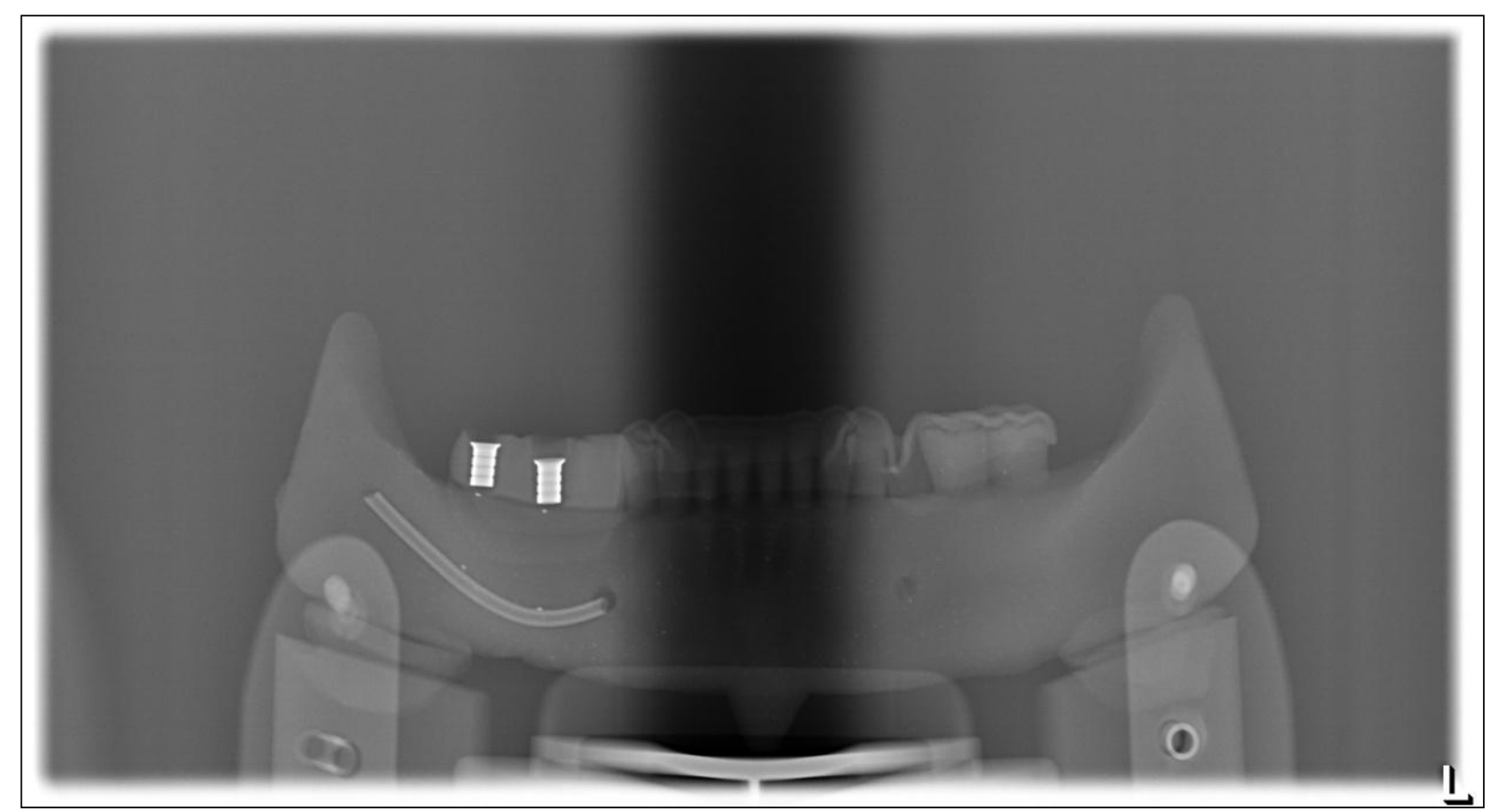

(b)

Abb. 13a: Markerschablone vom Typ „Hülse“ auf Röntgenmodell (a) und entsprechende Panoramaschichtaufnahme (b) 


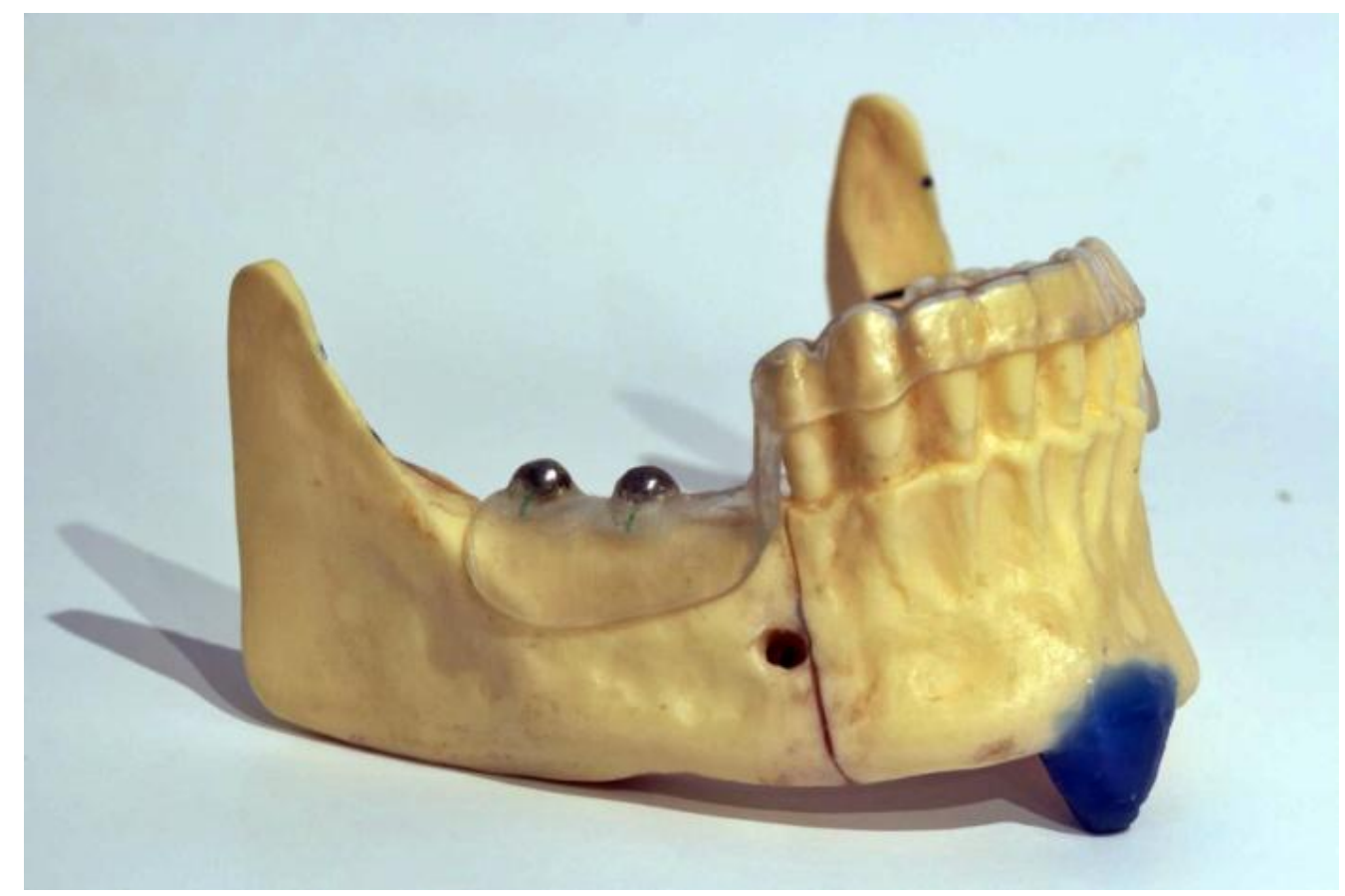

(a)

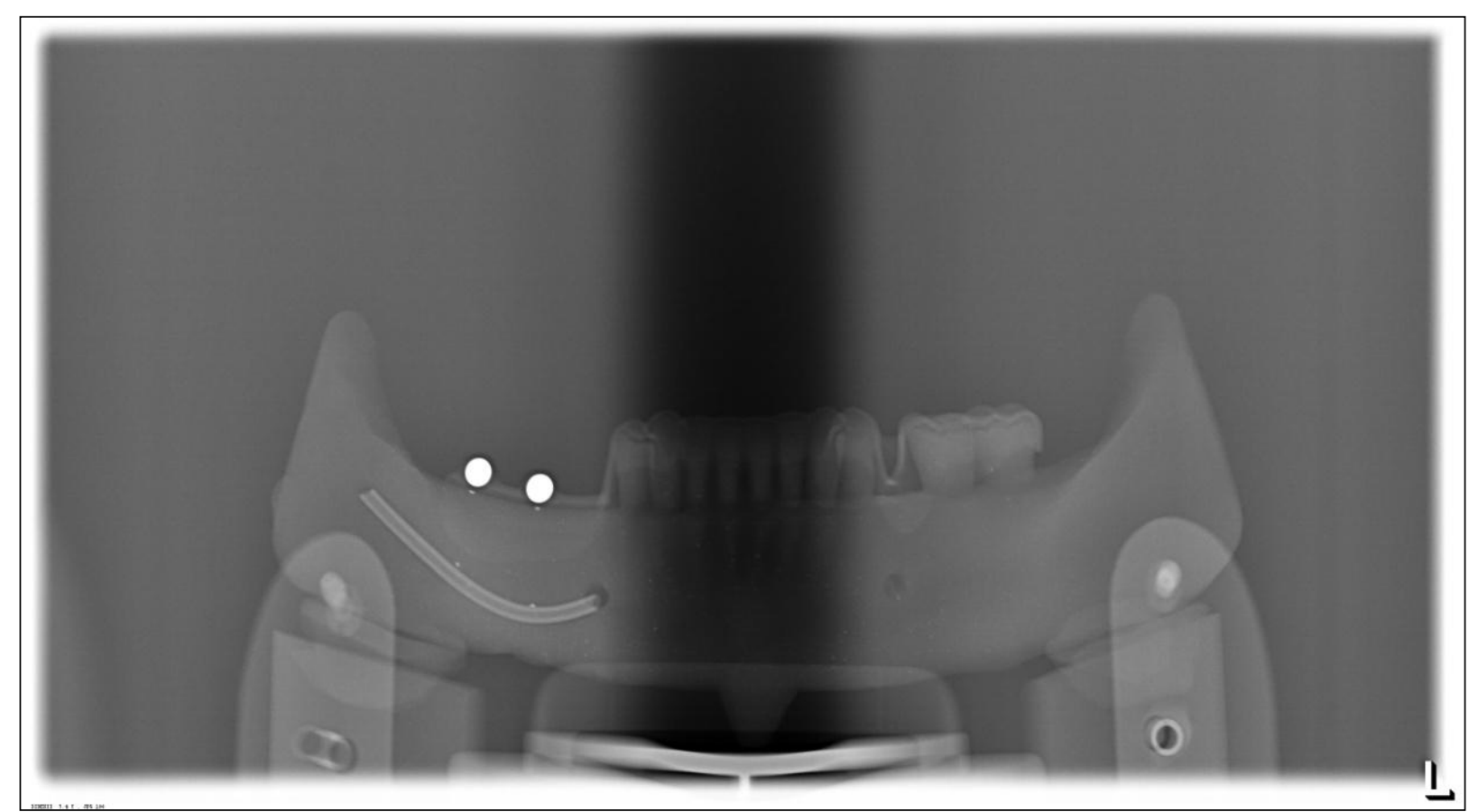

(b)

Abb. 14: Markerschablone vom Typ „Kugel“ auf Röntgenmodell (a) und entsprechende Panoramaschichtaufnahme (b) 


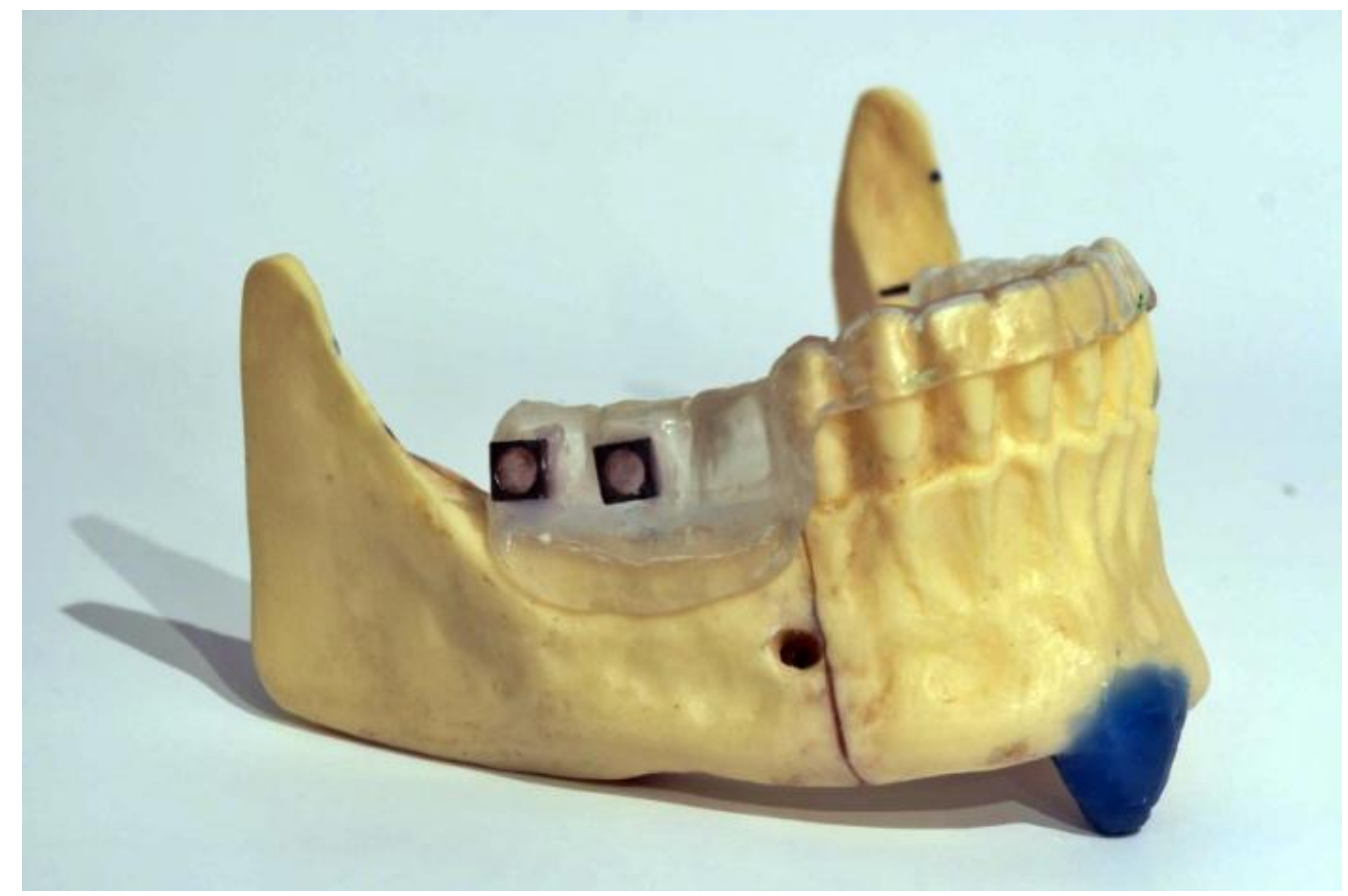

(a)

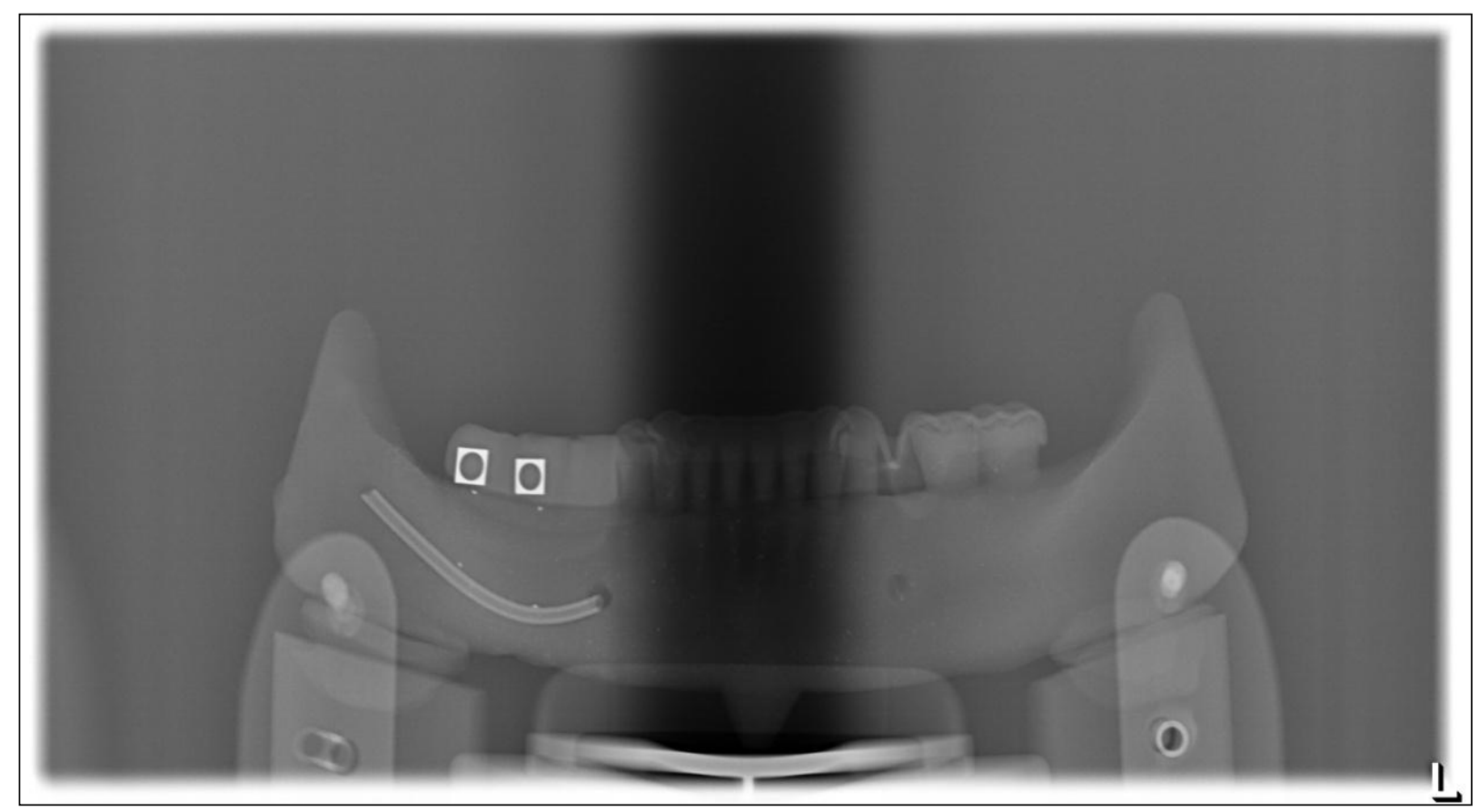

(b)

Abb. 15: Markerschablone vom Typ „Plättchen“ auf Röntgenmodell (a) und entsprechende Panoramaschichtaufnahme (b) 


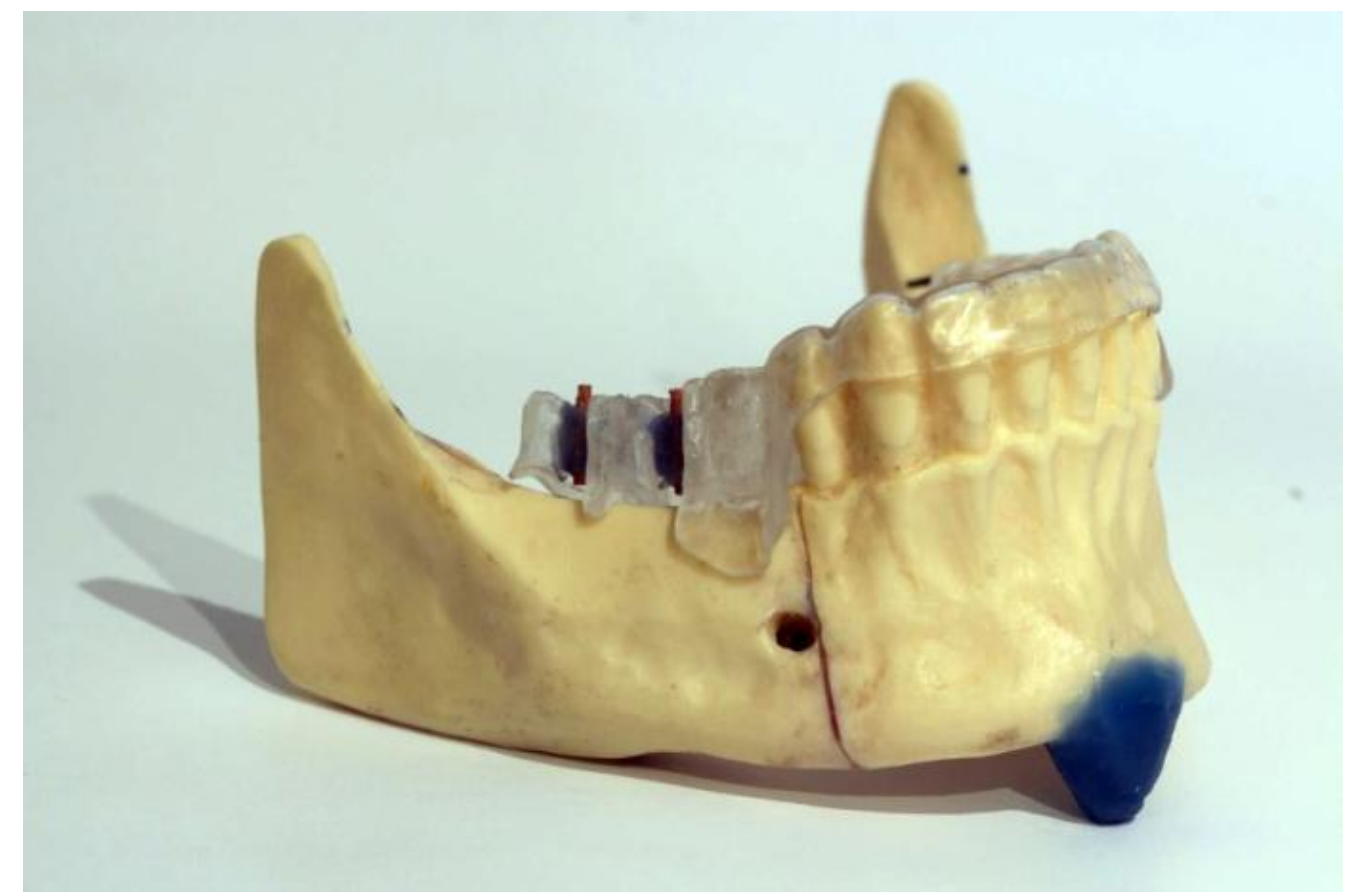

(a)

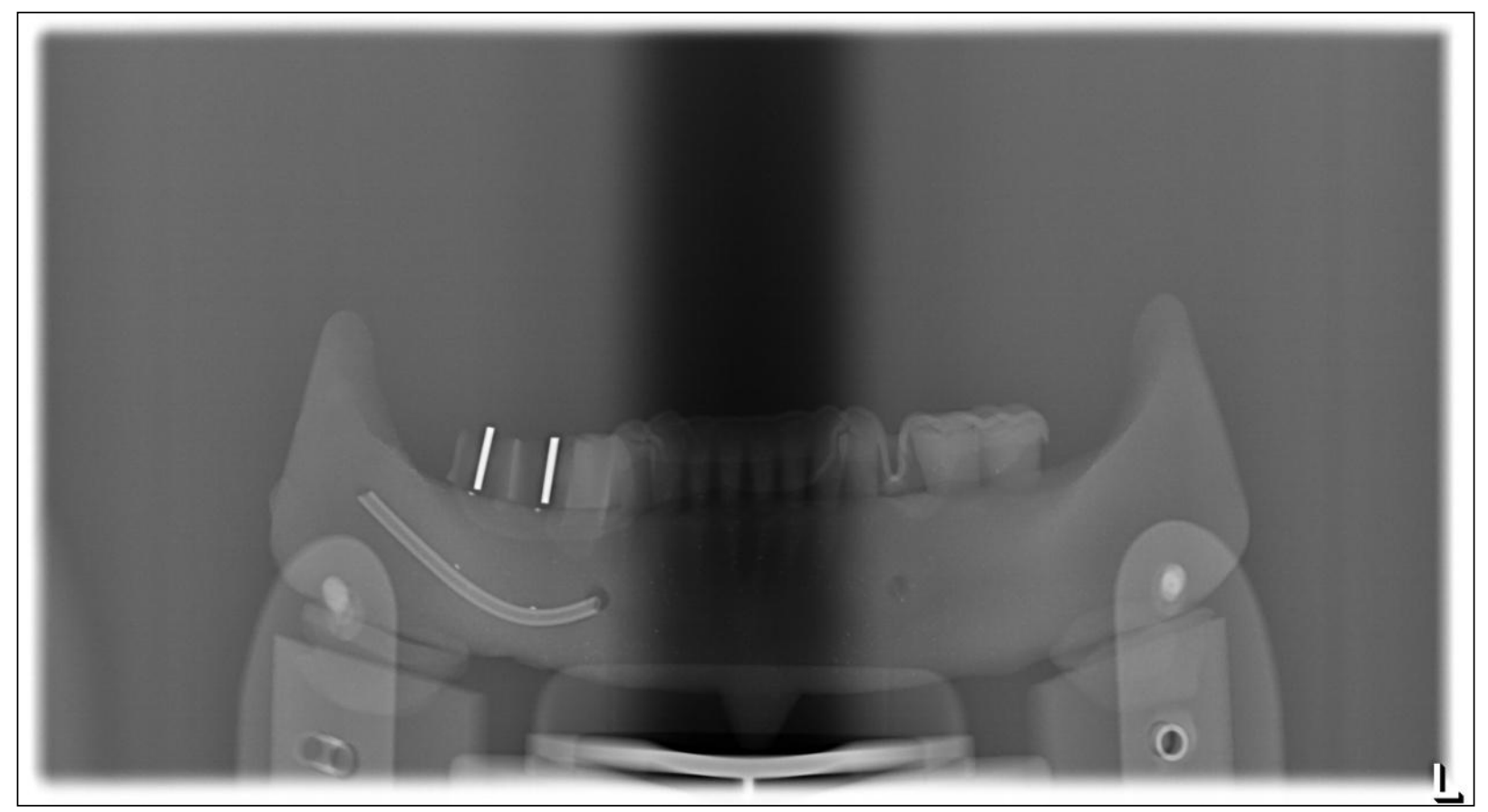

(b)

Abb. 16: Markerschablone vom Typ „Point“ auf Röntgenmodell (a) und entsprechende Panoramaschichtaufnahme (b) 


\subsection{Vergleich der verschiedenen Markersysteme}

\subsubsection{Randomisation}

Die Versuchsplanung erfolgte in Zusammenarbeit mit der Abteilung Medizinische Statistik der Universitätsklinik Göttingen. Es wurde eine Stichprobengröße von 10 Probanden je gleicher Vermessung je Versuchsanordnung festgelegt.

$\mathrm{Da}$ vier Panoramaschichtaufnahmen vermessen werden sollten, wurde eine verbindliche Teilnehmerliste von 40 Probanden, 20 Frauen und 20 Männern, erstellt. Hierbei handelte es sich um Zahnmedizinstudenten des 9. Semesters. Die Zuordnung der Probanden zu den vier Markergruppen erfolgte durch einfache, nach Geschlecht stratifizierte Randomisation. Je fünf Männer und fünf Frauen wurden zufällig einer Markergruppe zugeordnet. So war gewährleistet, dass jeder Proband nur eine Panoramaschichtaufnahme vermaß, um einen Lerneffekt zu vermeiden. Die Probanden wurden im Verlauf einer Woche einzeln zur Vermessung gerufen. Ihnen wurde untersagt, mit anderen Versuchsteilnehmern über die Ergebnisse zu sprechen, um eine gegenseitige Beeinflussung möglichst gering zu halten. Der Versuchsaufbau war für alle Probanden gleich angeordnet, um identische Messvoraussetzungen sicherzustellen.

\subsubsection{Versuchsablauf}

Die vier verschiedenen Panoramaschichtaufnahmen wurden jeweils von 10 Probanden vermessen. Dazu standen eine digitale Schieblehre (Anzeigegenauigkeit 0,01 mm; Messgenauigkeit 0,03 mm) und ein Geometriedreieck (Rotring GeoDreieck mit Griff 16 cm R823042) zur Verfügung. Nach jeder Messung wurde die digitale Schieblehre auf null kalibriert. 
Zusammenfassung des Versuchsablaufes:

- Es gibt vier verschiedene Markersysteme („Hülse“, „Kugel“, „Plättchen“ und „Point"), für welche jeweils eine Tiefziehschablone angefertigt wurde.

- Mit jeder der vier Tiefziehschablonen mit ihrem entsprechenden Markersystem wurde eine Röntgenaufnahme in identischer Aufnahmetechnik auf einem künstlichen Unterkiefer angefertigt und diese auf Thermoprintfolien ausgedruckt.

- Jedes der vier Röntgenbilder wurde von jeweils 10 Versuchsteilnehmern (fünf Männer und fünf Frauen) auf einem Lichtkasten (DIN 6856-1) vermessen.

- Die Strecken wurden von den Probanden mit einem Geometriedreieck und anschließend mit einer elektrischen Schieblehre gemessen. Gemessen wurden: jeweils für zwei Implantatorte (Regio 46 und 47) die röntgenologische Knochenhöhe und die röntgenologische Markerhöhe.

- Zusätzlich maß die „Point“-Gruppe die reale Markerlänge des Guttaperchapoints, da dieser der einzige nicht standardisierte Marker war.

Die gemessenen Werte wurden von den Probanden diktiert und vom Versuchsleiter in einen Erfassungsbogen eingetragen. Dann wurde mit einem Taschenrechner der Abstand zwischen den Referenzpunkten aus Guttapercha errechnet. Dazu diente der Dreisatz gemäß:

$$
\begin{aligned}
& \frac{\left[\mathrm{h}_{\mathrm{K} \text { real }}\right]}{\left[\mathrm{h}_{\mathrm{M}_{\text {real }}}\right]}=\frac{\left[\mathrm{h}_{\mathrm{K} \text { rö }}\right]}{\left[\mathrm{h}_{\mathrm{M} \text { rö }}\right]} \quad \rightarrow\left[\mathrm{h}_{\mathrm{K} \text { real }}\right]=\frac{\left[\mathrm{h}_{\mathrm{K} \text { rö }}\right] *\left[\mathrm{~h}_{\left.\mathrm{M}_{\text {real }}\right]}\right.}{\left[\mathrm{h}_{\mathrm{M} \text { rö }}\right]} \\
& \text { Legende: } \quad h_{K \text { real }} \text { Höhe Knochen real } \\
& \mathrm{h}_{\mathrm{K} \text { rŏ }} \text { Höhe Knochen röntgenologisch } \\
& \mathrm{h}_{\mathrm{M} \text { real }} \text { Höhe Marker real } \\
& \mathrm{h}_{\text {M rö }} \text { Höhe Marker röntgenologisch }
\end{aligned}
$$

Der Abstand der röntgenopaken Referenzpunkte aus Guttapercha entspricht der real vorhandenen Knochenhöhe. Dieser Anstand kann direkt gemessen werden, da das Modell des Unterkiefers durch die Spaltung auseinandernehmbar ist. 


\subsubsection{Statistische Methoden}

Die statistische Auswertung der Ergebnisse erfolgte in Zusammenarbeit mit der Abteilung Medizinische Statistik der Universitätsmedizin Göttingen. Die erhobenen Messdaten wurden tabellarisch in das Programm Excel (Microsoft) übertragen. Das Signifikanzniveau wurde für alle Tests mit $\alpha=5 \%$ festgelegt. Die statistischen Analysen wurden mit der Software SAS (Version 9.1, SAS Institute) vorgenommen. Der Einfluss von Geschlecht, Markertyp, Region und Messwerkzeug auf die Differenz zwischen echter und gemessener Markerhöhe bzw. Knochenhöhe wurde jeweils mit einer mehrfaktoriellen Varianzanalyse, inklusive aller Wechselwirkungen untersucht. Im Falle signifikanter Wechselwirkungen wurden die Varianzanalysen nach den Stufen der jeweiligen Einflussparameter aufgetrennt. Für die anschließenden Paarvergleiche wurden ebenfalls Varianzanalysen verwendet.

\subsection{Vergleich Panoramaschichttechnik und digitale Volumentomographie}

Die im Versuch 2.1.9 erstellte Panoramaschichtaufnahme mit der Kugelmarkerschablone und eine entsprechende Aufnahme des Unterkiefermodells mit Kugelmarkerschablone im digitalen Volumentomographen NewTom 9000 wurden auf ihre Genauigkeit bei der Bestimmung des vertikalen Knochenangebotes gegenübergestellt. Dazu wurden jeweils die vorhandenen Knochenhöhen in Regio 46 und 47 mit den jeweiligen programmintegrierten Messwerkzeugen am Computer vermessen.

\subsubsection{Erstellen einer DVT-Aufnahme}

Um das Röntgenmodell mit den jeweiligen Planungsschablonen im digitalen Volumentomograph vermessen zu können, musste zunächst eine Methode entwickelt werden, um die Röntgendichte des zu vermessenden Unterkiefermodells zu erhöhen. Eine Sicherung des Volumentomographen verhinderte, dass mit einer Spannung unter $110 \mathrm{kV}$ und einer Stromstärke unter 1,5 mA die Röntgenvorrichtung für ein dann unzureichend beurteilbares Bild auslöst. 
Um diesen Schutz zu umgehen, wurde das Unterkiefermodell am Boden eines Plexiglaszylinders (Höhe: $300 \mathrm{~mm}$, Durchmesser: $110 \mathrm{~mm}$, Wandstärke: $5 \mathrm{~mm}$ ) mit Hilfe von Silaplast befestigt. Dieser Zylinder wurde in einen mit vier Litern Leitungswasser gefüllten Eimer (Gesamtvolumen 10 I) gestellt.

Der Kunststoff des Röntgenmodells hatte eine nur gering höhere Röntgendichte als Wasser. Daher konnte es nicht einfach am Boden eines mit Wasser gefülten Gefäßes befestigt werden. Um eine ausreichende Röntgendichte von frontal zu erreichen, diente zusätzlich ein mit $500 \mathrm{ml}$ Wasser gefüllter Kunststoffbeutel, der direkt unterhalb des Wasserspiegels verschlossen wurde. Dieser wurde auf dem Modell im Zylinder platziert.

Die Aufnahmen wurden mit dem digitalen Volumentomographen NewTom 9000 der Röntgenabteilung des Zentrums für Zahn-, Mund- und Kieferheilkunde im Universitätsklinikum Göttingen erstellt. Dazu diente die Software NEWTOM (QR-DVT 9000 Version 3,37/NT). Die Kugelmarkerschablone wurde auf das Unterkiefermodell gesteckt und dieses wie oben beschrieben in der Versuchsanordnung platziert.

Mit Hilfe des Lichtvisiers wurde das Unterkiefermodell mittig im Röntgengerät ausgerichtet und die Aufnahme erstellt. Dabei stellten die Sensoren des NewTom automatisch Belichtungsparameter von $110 \mathrm{kV}$ und $17 \mathrm{~mA}$ ein.

\subsubsection{Vermessung der DVT-Aufnahme}

Die Schichtdicke beim hier verwendeten digitalen Volumentomographen NewTom 9000 kann bei der dreidimensionalen Rekonstruktion zwischen 0,3 mm, 0,5 mm und $1 \mathrm{~mm}$ gewählt werden. Die anschließende zweidimensionale Rekonstruktion kann in Schichtdicken von $0,5 \mathrm{~mm}, 1 \mathrm{~mm}, 2 \mathrm{~mm}, 3 \mathrm{~mm}$ und $5 \mathrm{~mm}$ durchgeführt werden (Software NEWTOM; QR-DVT 9000-study).

Zur Auswertung erfolgte eine computergestützte Rekonstruktion (primary reconstruction) mit einer axialen Schichtstärke von $0,3 \mathrm{~mm}$ (high resolution). Der daraus gewonnene dreidimensionale Datensatz des Röntgenmodells mit den jeweiligen Markertypen konnte anschließend mit einem im Programm integrierten Messwerkzeug ausgemessen werden. Dazu wurden in Aufsicht auf den Unterkieferbogen im Bereich der Röntgenmarker virtuelle Schnittbilder in einem Abstand von 0,5 mm, senkrecht zum Verlauf des Kieferkamms erstellt (secondary 
seconstruction $\rightarrow$ transaxials sections setup). Auf den berechneten Einzelbildern (sequenz of transaxial sections) konnte anhand der eingeschmolzenen Guttaperchapunkte die Knochenhöhe bestimmt werden.

Es wurde ein sagittales Schnittbild ausgewählt, auf dem alle vier Guttaperchahalbkugeln gut erkennbar waren. Dieses wurde mit dem programminternen Lupentool (zoom image) maximal vergrößert.

Zur Vermessung des digitalen Volumentomogramms wurden aus dem Pool der Versuchsteilnehmer (Versuch 2.2.1) 10 Studenten der Zahnmedizin bestimmt, fünf weibliche und fünf männliche.

Anschließend wurde das Bild von den 10 Versuchsteilnehmern nacheinander vermessen. Dazu diente das Messwerkzeug unter distances toolbar $\rightarrow$ new distance.

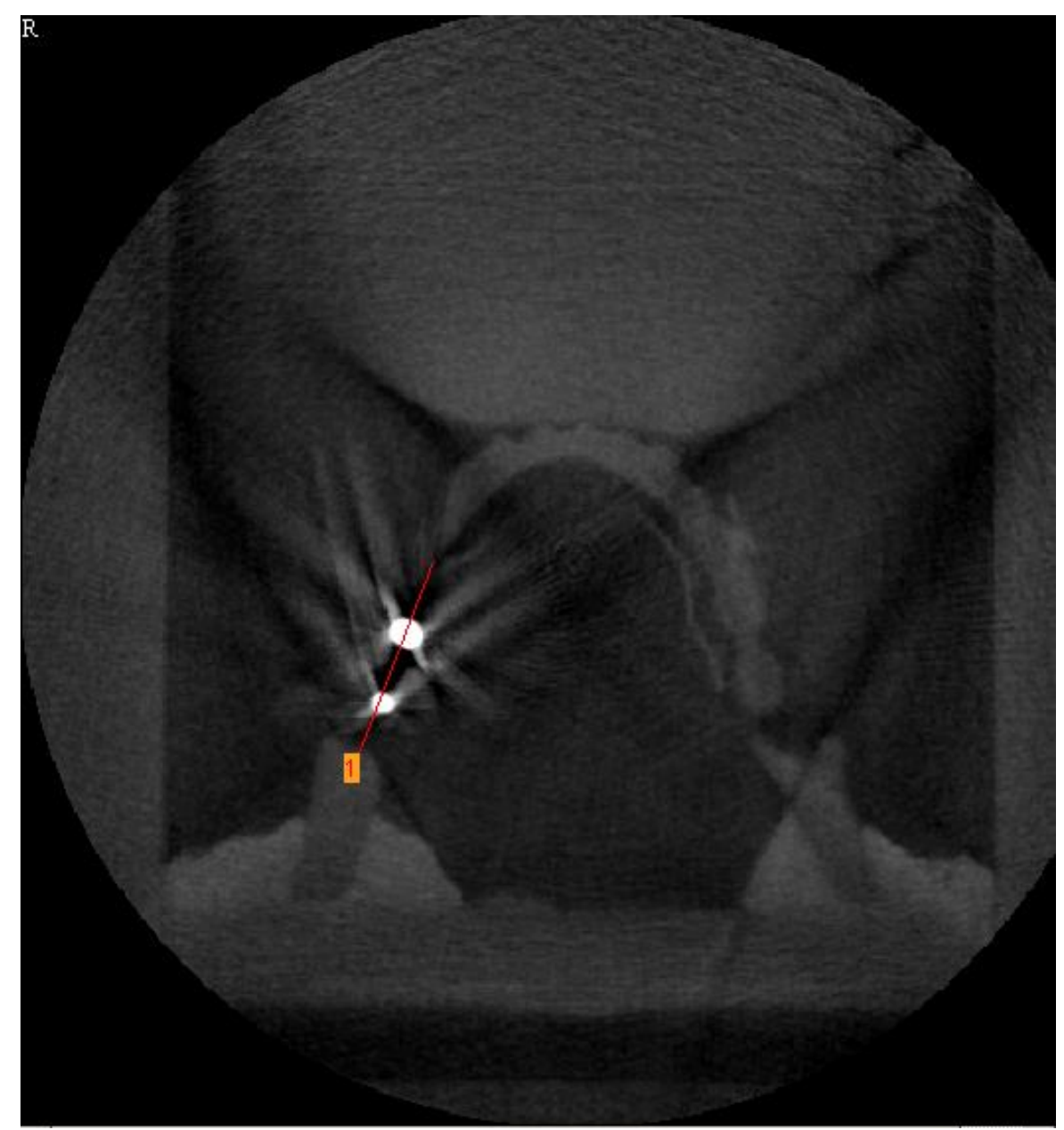

Abb. 17: Sagittaler Schnitt durch die crestal platzierten Kugelmarker auf dem Unterkiefermodell 


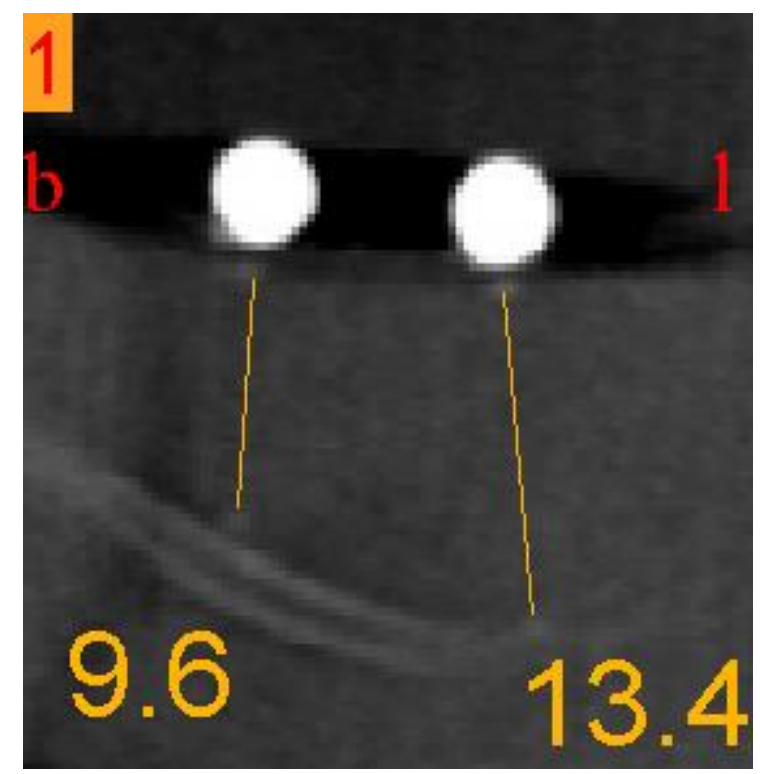

Abb. 18: Vermessung der Vertikalen Knochenhöhe nach sekundärer Rekonstruktion bei allen in einer Schicht getroffenen Guttaperchamarkern

\subsubsection{Vermessung der Panoramaschichtaufnahme}

Beim Orthophos XG Plus erfolgte die Bilddarstellung und Vermessung mit der Software „SIDEXIS neXt Generation 2.4“. Zunächst wurde mit dem Werkzeug Lupe der zu untersuchende Bereich im geöffneten Orthopantomogramm vergrößert dargestellt. Daraufhin musste mit dem, im Programm integrierten Messwerkzeug unter dem Menüpunkt Analyse $\rightarrow$ Länge messen, der Kugelmarker in Implantationsrichtung in seiner vertikalen Ausdehnung gemessen werden. Vor der eigentlichen Messung der Knochenhöhe konnte bei diesem Programm der Vergrößerungsfaktor der Panoramaschichtaufnahme über den Kugelmarker kalibriert werden. Dazu wurde im Menüpunkt Analyse $\rightarrow$ Länge messen $\rightarrow$ Maße anpassen die tatsächliche Länge auf $4 \mathrm{~mm}$ eingestellt (dies entspricht dem realen Durchmesser des Kugelmarkers). Die röntgenologische Knochenhöhe wurde durch Benutzen des Längenmesswerkzeuges unter dem Menüpunkt Analyse $\rightarrow$ Länge messen als kürzester Abstand zwischen den jeweiligen Verschattungen im Sinne der im Modell eingeschmolzenen Guttapercha-Viertelkugeln definiert. Der ermittelte Wert sollte durch den vorher vom Versuchsleiter durchgeführten Kalibriervorgang dem im Modell entsprechenden realen vertikalen Knochenangebot entsprechen. 
Die Vermessung der Panoramaschichtaufnahme erfolgte durch dieselben 10 Versuchsteilnehmer wie bei der DVT-Aufnahme (Versuch 2.3.2).

Für die Auswertung stand innen das Messwerkzeug unter dem Menüpunkt Analyse $\rightarrow$ Länge messen und die wie oben beschriebene auf der Computer-Arbeitsfläche vergrößerte Röntgenaufnahme zur Verfügung. Die Aufgabenstellung lautete, den kürzesten Abstand zwischen den röntgendichten Verschattungen der eingeschmolzenen Guttapercha-Viertelkugeln zu messen. Eine zufällige Zuordnung wie in Versuch 2.2 war nicht notwendig, da alle Versuchsteilnehmer dasselbe Bild vermessen sollten.

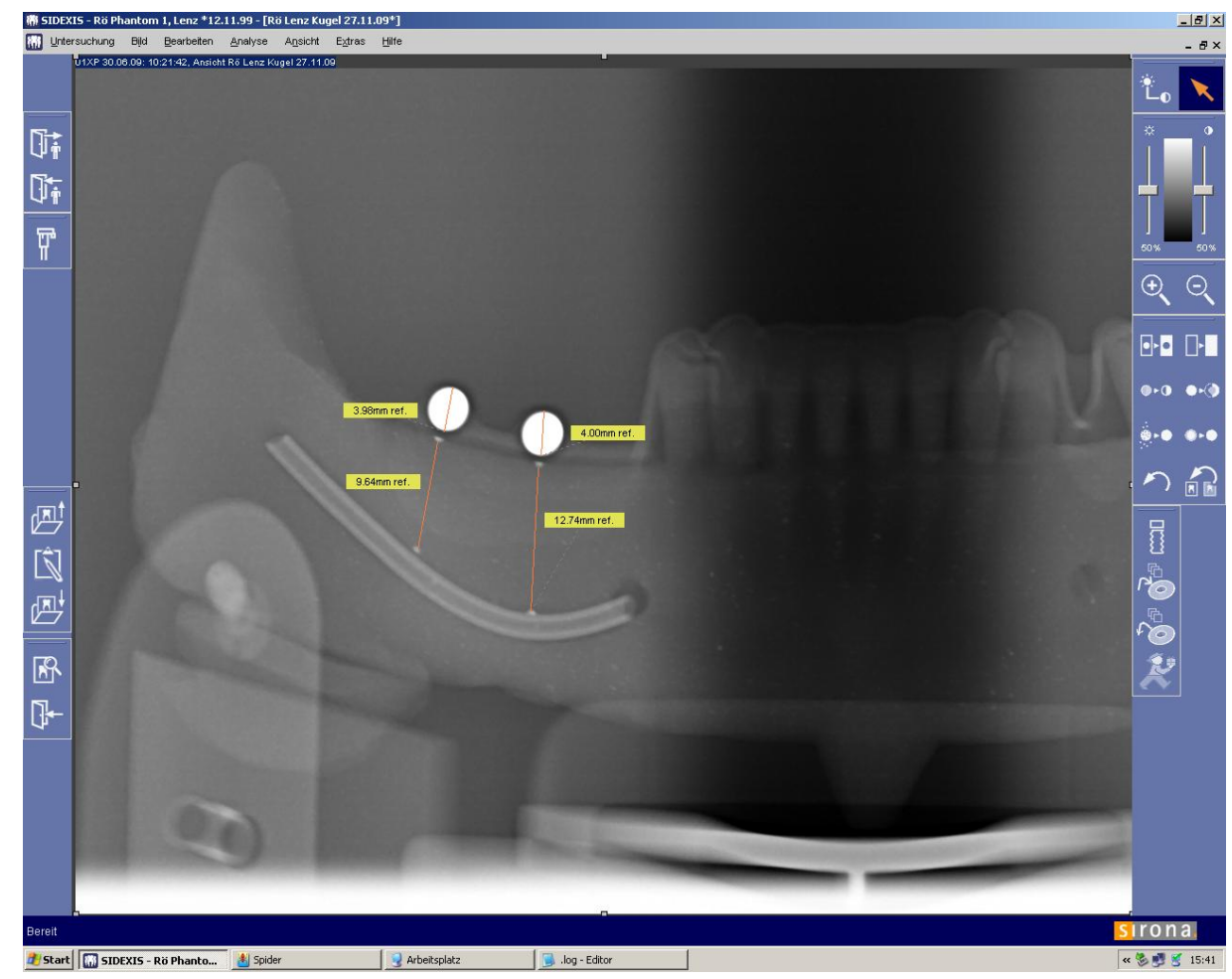

Abb. 19: Arbeitsoberfläche der Software SIDEXIS neXt Generation 2.4 des Orthophos XG Plus. Exemplarische Messung der Kugelmarker mit kalibriertem Vergrößerungsfaktor im Implantationsprogramm 


\subsubsection{Statistische Methoden}

Die statistische Auswertung der Ergebnisse erfolgte in Zusammenarbeit mit der Abteilung Medizinische Statistik der Universitätsmedizin Göttingen. Die erhobenen Messdaten wurden tabellarisch in das Programm Microsoft Excel übertragen. Die Analysen wurden mit der freien Software $\mathrm{R}$ (Version 2.8, www.r-project.org) vorgenommen. Das Signifikanzniveau wurde auf $5 \%$ festgesetzt. Der Einfluss des Messwerkzeuges und der Region auf die Messabweichung wurde mit einer zweifaktoriellen Varianzanalyse untersucht. Innerhalb jeder Region wurde nochmal ein Vergleich der Messwerkzeuge mit einem t-Test vorgenommen.

Die Darstellung der Ergebnisse der Varianzanalysen erfolgte tabellarisch. Hierbei wurden die einzelnen Einflussfaktoren und die Gruppen untereinander auf ihre statistische Signifikanz hin untersucht. 


\section{Ergebnisse}

Die geplante Stichprobengröße wurde erreicht. Die Probanden waren kooperativ. Die Vermessung der Röntgenaufnahmen verlief, wie geplant.

\subsection{Ergebnisse für die verschiedenen Markersysteme}

\subsubsection{Messung aller Marker}

Weder Geschlecht, noch Messwerkzeug hatten einen signifikanten Einfluss auf das Messergebnis. Daher wurden die Ergebnisse in den Boxplots zusammengefasst.

Die crestal platzierten Hülsen- und Kugelmarker standen im jeweiligen Röntgenbild genau über der Implantationsregion. Der Hülsenmarker in Regio 46 erschien auf den Röntgenaufnahmen gekippt. In der zweidimensionalen Abbildung zeigten sich dadurch Doppellinien am oberen und unteren Rand (Abb. 20).
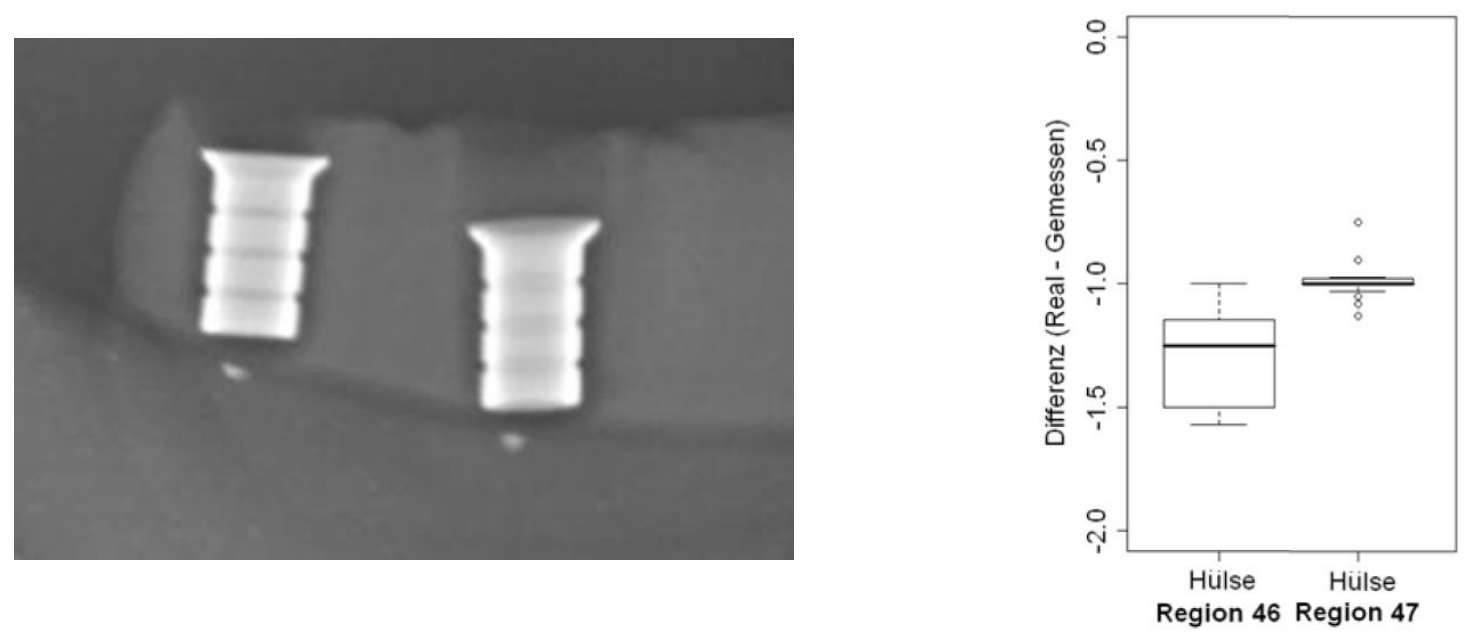

Abb. 20: Vergrößerter Ausschnitt des OPGs (links) und Differenz der im Bild gemessenen Höhe der Hülsenmarker (rechts) zur realen Höhe $(n=10)$

Die Abbildungen der Kugeln stellten sich nicht perfekt rund, sondern leicht hochoval dar (siehe Abb. 21). 

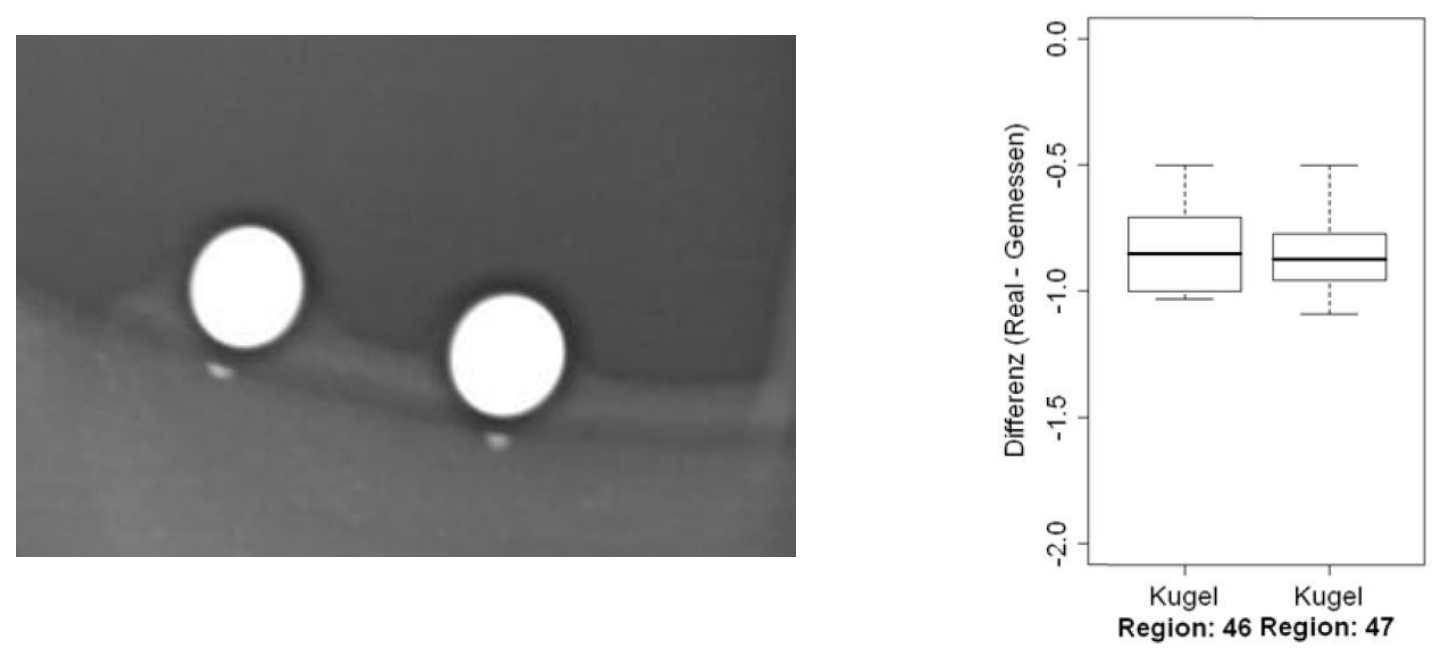

Abb. 21: Vergrößerter Ausschnitt des OPGs (links) und Differenz der im Bild gemessenen Höhe der Kugelmarker (rechts) zur realen Höhe $(n=10)$

Auch die quadratischen Plättchenmarker zeigten in ihrer Abbildung auf der Panoramaschichtaufnahme eine Verzeichnung (vergleiche Abb. 22).

Die Abbildungen der vestibulär stehenden Plättchenmarker projizierten sich im Röntgenbild nicht genau über den Guttaperchamarkern, welche Implantationsregionen definieren. So erschienen die beiden Plättchenmarker weiter distal verschoben auf der Panoramaschichtaufnahme. Dies verdeutlicht der vergrößerte Ausschnitt in Abb. 22.
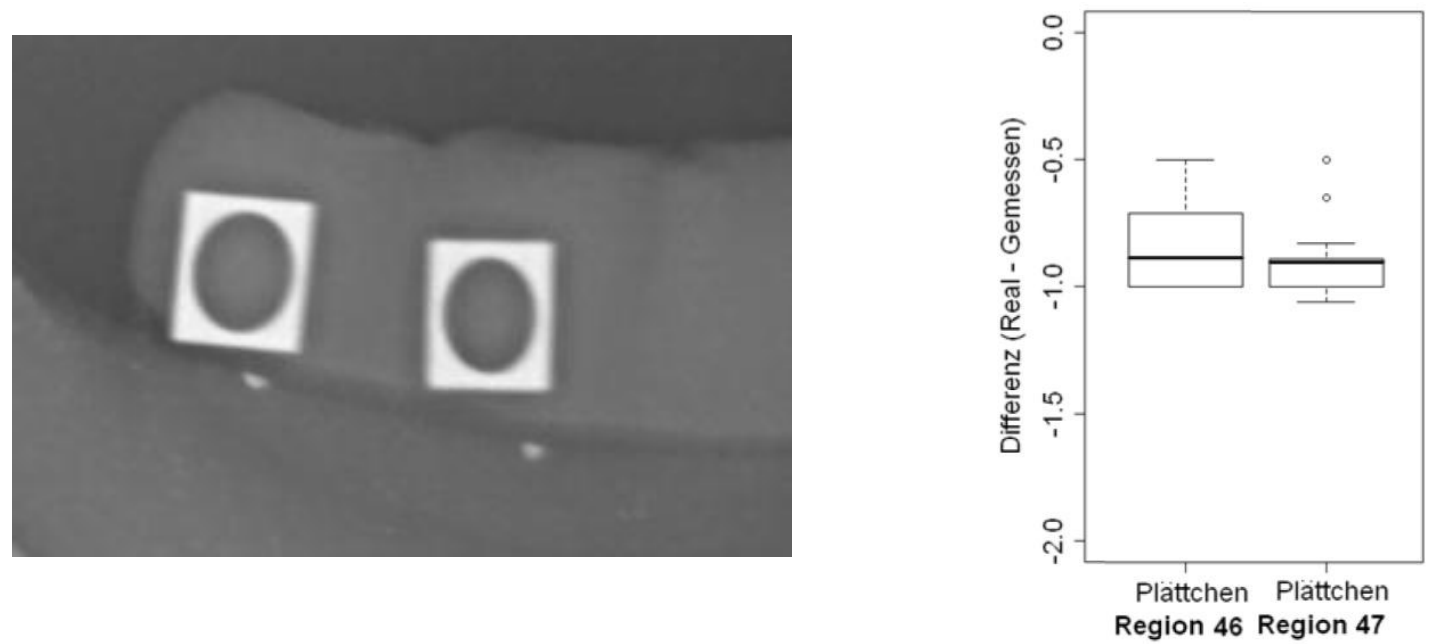

Abb. 22: Vergrößerter Ausschnitt des OPGs (links) und Differenz der im Bild gemessenen Höhe der bukkalen Plättchenmarker (rechts) zur realen Höhe $(n=10)$

Auch die Abbildungen der oral stehenden Pointmarker projizierten sich im Röntgenbild nicht genau über den Guttaperchamarkern, welche die Implantationsregionen 
definieren. So erschienen die beiden Pointmarker weiter mesial verschoben auf der Panoramaschichtaufnahme. Dies verdeutlicht der vergrößerte Ausschnitt in Abb. 23.
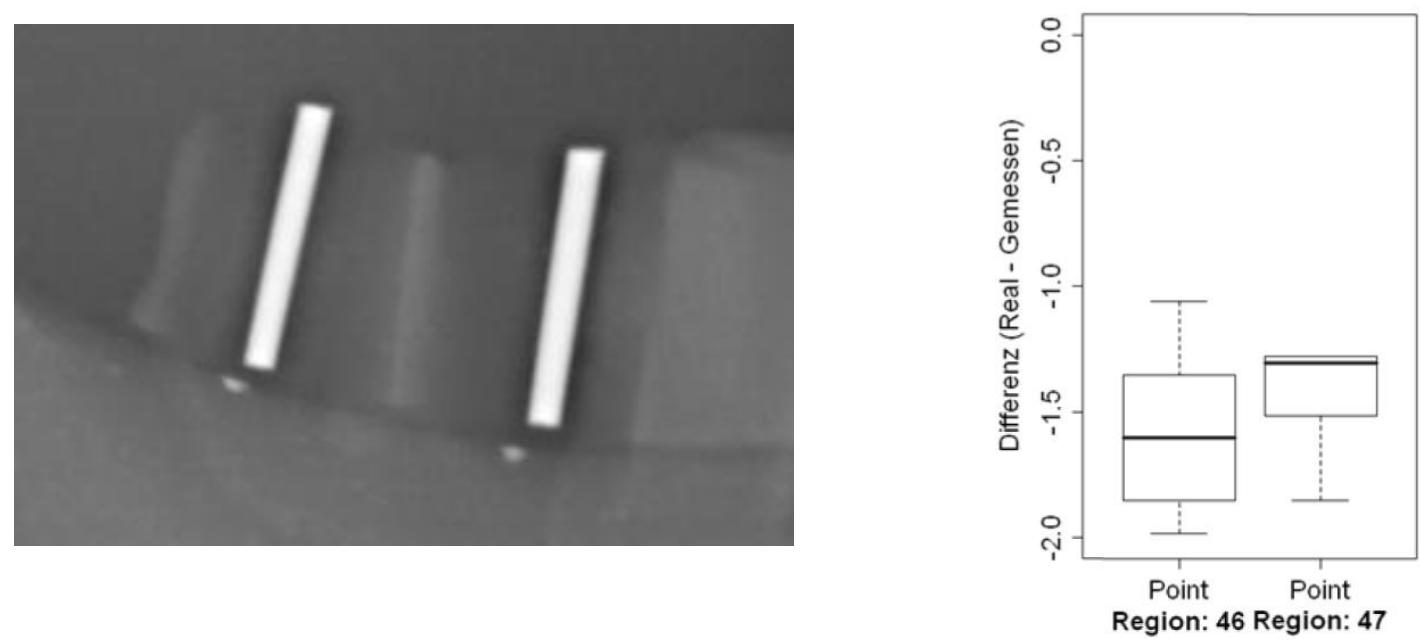

Abb. 23: Vergrößerter Ausschnitt des OPGs (links) und Differenz der im Bild gemessenen Höhe der lingualen Pointmarker (rechts) zur realen Höhe $(n=10)$

Die größte Differenz zwischen realer und gemessener Markerhöhe zeigte sich beim Pointmarker, die kleinste bei der Kugel (Tab. 4). Bei zunehmender Markerlänge nahm auch die Differenz zur realen Markerlänge zu.

Tab. 4: Mittelwert und Standardabweichung der Differenzen zwischen realer und gemessener Markerhöhe, getrennt nach Markertyp und Region

\begin{tabular}{|c|c|c|}
\hline \multirow[t]{2}{*}{ Marker } & \multicolumn{2}{|l|}{ Region } \\
\hline & 46 & 47 \\
\hline Hülse & $-1.29 \pm 0.20$ & $-0.98 \pm 0.08$ \\
\hline Kugel & $-0.84 \pm 0.15$ & $-0.84 \pm 0.16$ \\
\hline Plättchen & $-0.84 \pm 0.16$ & $-0.90 \pm 0.13$ \\
\hline Point & $-1.57 \pm 0.29$ & $-1.41 \pm 0.17$ \\
\hline
\end{tabular}

Bei den Analysen zeigte sich ein signifikanter Einfluss von Markertyp und Region bei der Differenz der gemessenen Markerhöhen zu den realen Abmessungen (Tab. 5). Außerdem konnten Wechselwirkungen zwischen den beiden Faktoren Markertyp und Region festgestellt werden. 
Tab. 5: Ergebnisse der Varianzanalyse für die Differenz zwischen realer und gemessener Markerhöhe

\begin{tabular}{|l|l|r|}
\hline Effekte & \multicolumn{1}{|c|}{ p } \\
\hline Haupteffekte & Geschlecht & 0.75 \\
\cline { 2 - 3 } & Marker & $<\mathbf{0 . 0 1}$ \\
\cline { 2 - 3 } & Region & $\mathbf{0 . 0 1}$ \\
\cline { 2 - 3 } & Messwerkzeug & 0.85 \\
\hline \multirow{5}{*}{ Wechselwirkungen } & Geschlecht x Marker & 0.38 \\
\cline { 2 - 3 } & Geschlecht x Region & 0.54 \\
\cline { 2 - 3 } & Geschlecht x Messwerkzeug & 0.85 \\
\cline { 2 - 3 } & Marker x Region & $<\mathbf{0 . 0 1}$ \\
\cline { 2 - 3 } & Marker x Messwerkzeug & 0.18 \\
\cline { 2 - 3 } & Region x Messwerkzeug & 0.31 \\
\cline { 2 - 3 } & Geschlecht x Marker x Region & 0.85 \\
\cline { 2 - 3 } & Geschlecht x Marker x Messwerkzeug & 0.26 \\
\cline { 2 - 3 } & Geschlecht x Region x Messwerkzeug & 0.26 \\
\cline { 2 - 3 } & Marker x Region x Messwerkzeug & 0.09 \\
\cline { 2 - 3 } & Geschecht x Marker x Region x Messwerkzeug & 0.45 \\
\hline
\end{tabular}

Um eine Überschneidung dieser Einflussfaktoren auszuschließen, wurde die Varianzanalyse für beide Regionen getrennt vorgenommen (Tab. 6). Die Ergebnisse der Varianzanalyse zeigten, dass die Marker wieder einen signifikanten Einfluss auf die Differenz der realen zur gemessenen Markerhöhe bzw. die Differenz der realen zur gemessenen Knochenhöhe hatten.

Tab. 6: Ergebnisse der Varianzanalyse für die Differenz der Markerhöhe, getrennt nach Region

\begin{tabular}{|c|c|c|c|}
\hline \multirow{2}{*}{$\begin{array}{l}\text { Region } \\
46\end{array}$} & \multicolumn{2}{|l|}{ Effekte } & \multirow{2}{*}{$\begin{array}{ll}\mathbf{p} & \\
& 0.57\end{array}$} \\
\hline & \multirow[t]{3}{*}{ Haupteffekte } & Geschlecht & \\
\hline & & Marker & $<0.01$ \\
\hline & & Messwerkzeug & 0.31 \\
\hline & \multirow[t]{4}{*}{ Wechselwirkungen } & Geschlecht x Marker & 0.73 \\
\hline & & Geschlecht x Messwerkzeug & 0.56 \\
\hline & & Marker x Messwerkzeug & 0.05 \\
\hline & & Geschlecht x Marker x Messwerkzeug & 0.19 \\
\hline \multirow[t]{7}{*}{47} & \multirow[t]{3}{*}{ Haupteffekte } & Geschlecht & 0.80 \\
\hline & & Marker & $<0.01$ \\
\hline & & Messwerkzeug & 0.75 \\
\hline & \multirow[t]{4}{*}{ Wechselwirkungen } & Geschlecht x Marker & 0.37 \\
\hline & & Geschlecht x Messwerkzeug & 0.26 \\
\hline & & Marker x Messwerkzeug & 0.73 \\
\hline & & Geschlecht x Marker x Messwerkzeug & 0.93 \\
\hline
\end{tabular}


Hinsichtlich des Regionenvergleichs war die Markerhöhendifferenz nur bei den Markertypen „Point“ und „Hülse“ verschieden (Abb. 20, Abb. 23 und Tab. 7).

Tab. 7: p-Werte für den Vergleich der Markerhöhendifferenz zwischen Region 46 und 47, getrennt nach Markertyp

\begin{tabular}{|c|c|c|c|c|}
\hline \multirow[t]{2}{*}{ Vergleich } & \multicolumn{4}{|l|}{ Marker } \\
\hline & Hülse & Kugel & Plättchen & Point \\
\hline 46 vs. 47 & $<0.01$ & 0.92 & 0.20 & 0.04 \\
\hline
\end{tabular}

Da die Marker einen signifikanten Einfluss auf die Differenz der Markerhöhe hatten, wurden die verschiedenen Marker paarweise gegenüberstellt. Tab. 8 zeigt die Ergebnisse. Hierbei ist zu beachten, dass in dieser Tabelle ein Signifikanzniveau von $\alpha=0.05 / 6=0.0083$ anzunehmen ist.

Tab. 8: p-Werte für die paarweisen Vergleiche der Differenz der Markerhöhe, getrennt nach Region (Signifikanz ist hier bei $p<0.05 / 6=0.0083$ gegeben)

\begin{tabular}{|l|r|r|}
\hline Vergleich & \multicolumn{2}{|l|}{ Region } \\
\cline { 2 - 4 } & $\mathbf{4 6}$ & $\mathbf{4 7}$ \\
\hline Hülse vs. Kugel & $<\mathbf{0 . 0 0 0 1}$ & $<\mathbf{0 . 0 0 0 1}$ \\
\hline Hülse vs. Plättchen & $<\mathbf{0 . 0 0 0 1}$ & 0.0193 \\
\hline Hülse vs. Point & $\mathbf{0 . 0 0 1 2}$ & $<\mathbf{0 . 0 0 0 1}$ \\
\hline Kugel vs. Plättchen & 0.9838 & 0.1754 \\
\hline Kugel vs. Point & $<\mathbf{0 . 0 0 0 1}$ & $<\mathbf{0 . 0 0 0 1}$ \\
\hline Plättchen vs. Point & $<\mathbf{0 . 0 0 0 1}$ & $<\mathbf{0 . 0 0 0 1}$ \\
\hline
\end{tabular}

Ausgehend von den Mittelwerten der gemessenen Längen wurden die mittleren lokalen Vergrößerungsfaktoren in den Panoramaschichtaufnahmen bestimmt (Tab. 9).

Tab. 9: Mittlere Vergrößerungsfaktoren, getrennt nach Markertyp und Region

\begin{tabular}{|c|c|c|}
\hline \multirow[t]{2}{*}{ Marker } & \multicolumn{2}{|l|}{ Region } \\
\hline & 46 & 47 \\
\hline Hülse & 1,18 & 1,16 \\
\hline Kugel & 1,21 & 1,21 \\
\hline Plättchen & 1,17 & 1,18 \\
\hline Point & 1,17 & 1,16 \\
\hline
\end{tabular}




\subsubsection{Messung Knochenhöhen}

Alle Probanden maßen die Knochenhöhe der Regionen 46 und 47 auf den ihnen vorgelegten Panoramaschichtaufnahmen aus. Da die Aufnahmen an demselben Modell und unter identischen Aufnahmebedingungen erstellt wurden, konnten die Daten zusammengefasst werden (Stichprobengröße $n=40$ ).

Beim Vergleich der beiden Regionen 46 und 47 zeigte sich eine Differenz zwischen realer und gemessener Knochenhöhe (Abb. 24, Tab. 10). Im Mittel war die Differenz in Regio 46 höher als in Regio 47 (Mittelwert \pm Standardabweichung: Regio 46: -2.2 \pm 0.4 ; Regio $47:-1.7 \pm 0.4$ ).

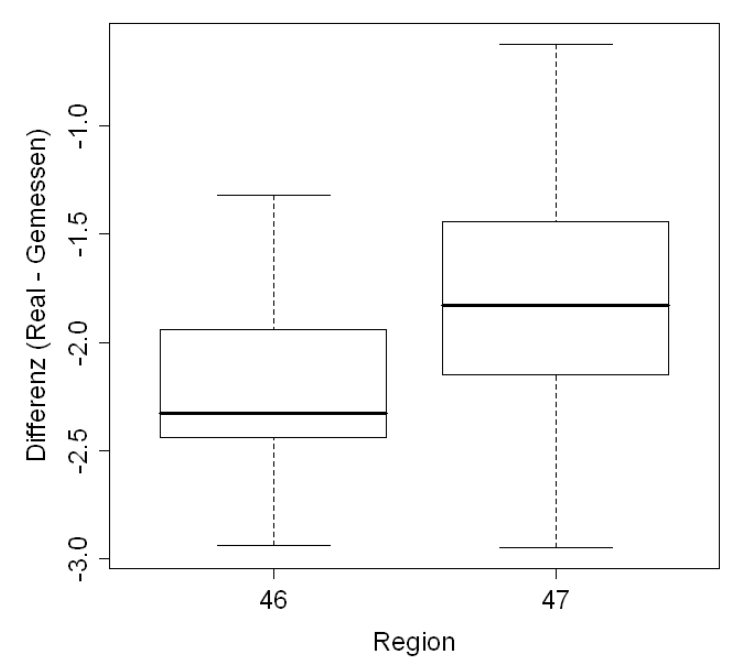

Abb. 24: Verteilungen der Differenzen zwischen realer und gemessener Knochenhöhe, getrennt nach Region $(n=40)$ 
Tab. 10: Ergebnisse der Varianzanalyse für die Differenz zwischen realer und in den

Panoramaschichtaufnahmen gemessenen Knochenhöhen

\begin{tabular}{|l|l|r|}
\hline Effekte & \multicolumn{1}{|c|}{ p } \\
\hline Haupteffekte & Geschlecht & 0.13 \\
\cline { 2 - 3 } & Marker & 0.61 \\
\cline { 2 - 3 } & Region & $\mathbf{0 . 0 1}$ \\
\cline { 2 - 3 } & Messwerkzeug & 0.23 \\
\hline Wechselwirkungen & Geschlecht x Marker & $\mathbf{0 . 0 4}$ \\
\cline { 2 - 3 } & Geschlecht x Region & 0.45 \\
\cline { 2 - 3 } & Geschlecht x Messwerkzeug & 0.54 \\
\cline { 2 - 3 } & Marker x Region & 0.90 \\
\cline { 2 - 3 } & Marker x Messwerkzeug & 0.54 \\
\cline { 2 - 3 } & Region x Messwerkzeug & 0.32 \\
\cline { 2 - 3 } & Geschlecht x Marker x Region & 0.91 \\
\cline { 2 - 3 } & Geschlecht x Marker x Messwerkzeug & 0.99 \\
\cline { 2 - 3 } & Geschlecht x Region x Messwerkzeug & 0.96 \\
\cline { 2 - 3 } & Marker x Region x Messwerkzeug & 0.95 \\
\cline { 2 - 3 } & Geschecht x Marker x Region x Messwerkzeug & 0.98 \\
\hline
\end{tabular}

Bei Betrachtung der röntgenologischen Knochenhöhe zur realen Knochenhöhe waren die Vergrößerungsfaktoren in Regio 46 und Regio 47 nahezu identisch (Tab. 11).

Tab. 11: Mittlerer Vergrößerungsfaktor Knochenhöhe getrennt nach Region

\begin{tabular}{|l|l|}
\hline Regio 46 & 1,17 \\
\hline Regio 47 & 1,18 \\
\hline
\end{tabular}




\subsubsection{Ergebnisse nach Kalibrierung}

Durch Messung der Markerhöhen in den Röntgenaufnahmen und die bekannten Abmessungen der Marker konnte eine Kalibrierung der Messungen in den Röntgenbildern mittels Dreisatz erfolgen (siehe Kapitel 2.2.2 S. 32).
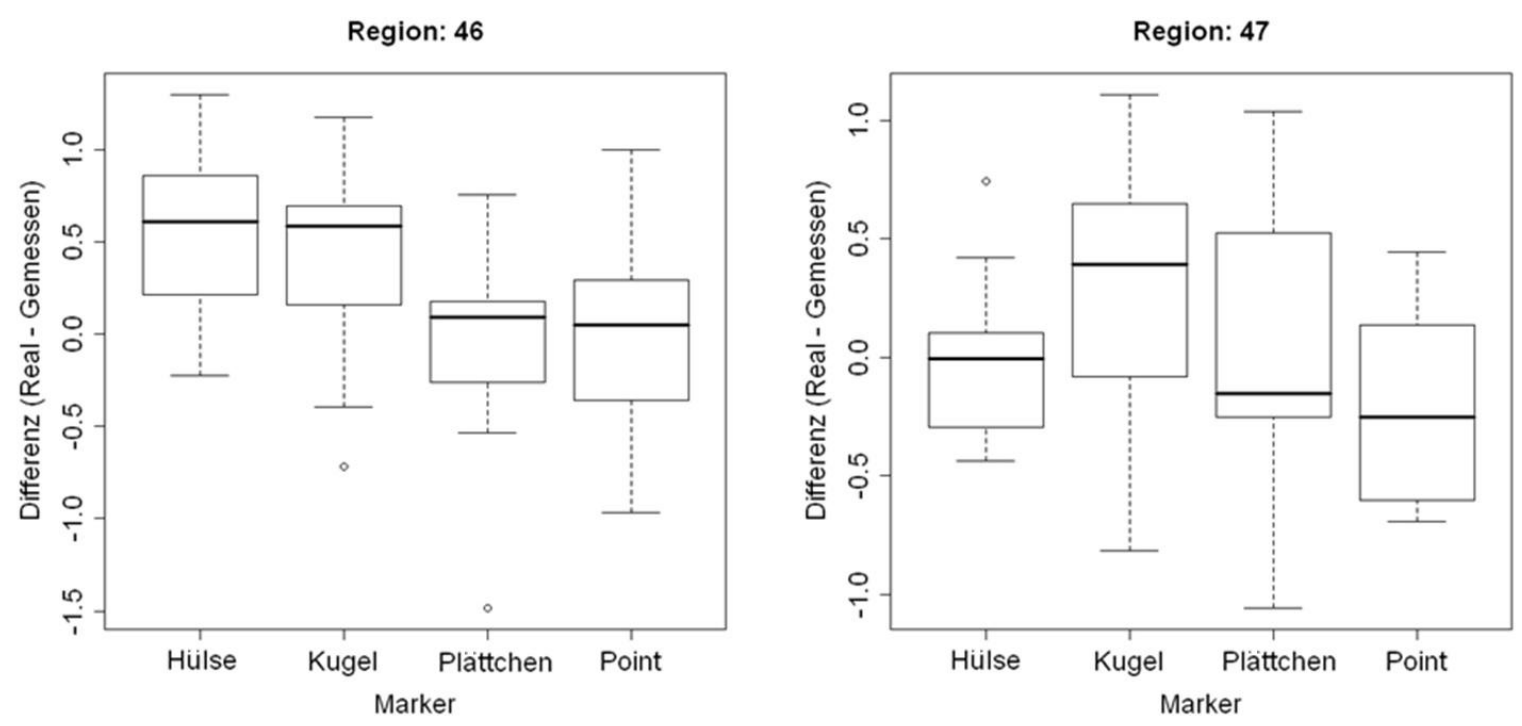

Abb. 25: Verteilungen der Differenzen (in $\mathrm{mm}$ ) zwischen realer und nach Kalibrierung gemessener Knochenhöhe, getrennt nach Markertyp und Region ( $n=10$ pro Marker)

Abb. 25 zeigt die Ergebnisse der durch den Dreisatz bestimmten Knochenhöhendifferenz (Kalibrierung). Hier zeigte die Hülse die stärkste Abhängigkeit vom Messort. Dies zeigt auch der Regionsvergleich bei dem die nach Kalibrierung bestimmte Knochenhöhe nur beim Markertyp Hülse signifikant vom realen Wert abwich (Tab. 12). Die Berechnung der Knochenhöhe mit Hilfe des leicht gekippten Hülsenmarkers in Regio 47 lag hier näher an der Höhe des realen Knochenangebotes. Die Kugel wies die höchste Differenz zwischen realer und nach Kalibrierung gemessener Knochenhöhe auf. Das Plättchen hatte im Mittel die geringste Differenz zwischen realer und nach Kalibrierung gemessener Knochenhöhe, jedoch eine im Vergleich hohe Standardabweichung. Der Point zeigte im Mittel geringe Differenzen, jedoch hohe Standardabweichungen. 
Tab. 12: p-Werte für den Vergleich der Differenzen der Knochenhöhen zwischen Region 46 und 47, getrennt nach Markertyp

\begin{tabular}{|c|c|c|c|c|}
\hline \multirow[t]{2}{*}{ Vergleich } & \multicolumn{4}{|l|}{ Marker } \\
\hline & Hülse & Kugel & Plättchen & Point \\
\hline 46 vs. 47 & $<0.01$ & 0.41 & 0.89 & 0.13 \\
\hline
\end{tabular}

Es konnten ein signifikanter Einfluss von Markertyp und Region der nach Kalibrierung bestimmten Knochenhöhendifferenz sowie eine Wechselwirkung zwischen diesen beiden Faktoren festgestellt werden (Tab. 13).

Tab. 13: Ergebnisse der Varianzanalyse für die Differenz zwischen echter und nach Kalibrierung ermittelter Knochenhöhe

\begin{tabular}{|l|l|r|}
\hline Effekte & p \\
\hline Haupteffekte & Geschlecht & 0.24 \\
\cline { 2 - 3 } & Marker & $<\mathbf{0 . 0 1}$ \\
\cline { 2 - 3 } & Region & $<\mathbf{0 . 0 1}$ \\
\cline { 2 - 3 } & Messwerkzeug & 0.72 \\
\hline Wechselwirkungen & Geschlecht x Marker & 0.47 \\
\cline { 2 - 3 } & Geschlecht x Region & 0.95 \\
\cline { 2 - 3 } & Geschlecht x Messwerkzeug & 0.97 \\
\cline { 2 - 3 } & Marker x Region & $\mathbf{0 . 0 5}$ \\
\cline { 2 - 3 } & Marker x Messwerkzeug & 0.49 \\
\cline { 2 - 3 } & Region x Messwerkzeug & 0.90 \\
\cline { 2 - 3 } & Geschlecht x Marker x Region & 0.81 \\
\cline { 2 - 3 } & Geschlecht x Marker x Messwerkzeug & 0.70 \\
\cline { 2 - 3 } & Geschlecht x Region x Messwerkzeug & 0.71 \\
\cline { 2 - 3 } & Marker x Region x Messwerkzeug & 0.68 \\
\cline { 2 - 3 } & Geschecht x Marker x Region x Messwerkzeug & 0.97 \\
\hline
\end{tabular}

Die Wechselwirkung bedeutet, dass der Markereffekt bei den beiden Regionen unterschiedlich ausgeprägt war bzw. dass der Regionseffekt bei den einzelnen Markern unterschiedlich ausgeprägt war. Daher wurde die Varianzanalyse für beide Regionen getrennt vorgenommen (Tab. 14) 
Tab. 14: Ergebnisse der Varianzanalyse für die Differenz der Knochenhöhe, die nach Kalibrierung ermittelt wurde, getrennt nach Region

\begin{tabular}{|l|l|l|r|}
\hline Region & Effekte & p \\
\hline 46 & Haupteffekte & Geschlecht & 0.45 \\
\cline { 3 - 4 } & & Marker & $<\mathbf{0 . 0 1}$ \\
\cline { 3 - 4 } & Messwerkzeug & 0.98 \\
\cline { 3 - 4 } & \multirow{3}{*}{ Wechselwirkungen } & Geschlecht x Marker & 0.74 \\
\cline { 3 - 4 } & & Geschlecht x Messwerkzeug & 0.78 \\
\cline { 3 - 4 } & & Marker x Messwerkzeug & 0.38 \\
\cline { 3 - 4 } & & Geschlecht x Marker x Messwerkzeug & 0.78 \\
\hline 47 & \multirow{3}{*}{ Haupteffekte } & Geschlecht & 0.36 \\
\cline { 3 - 4 } & & Marker & $\mathbf{0 . 0 1}$ \\
\cline { 3 - 4 } & & Messwerkzeug & 0.32 \\
\cline { 3 - 4 } & \multirow{2}{*}{ Wechselwirkungen } & Geschlecht x Marker & 0.86 \\
\cline { 3 - 4 } & & Geschlecht x Messwerkzeug & 0.89 \\
\cline { 3 - 4 } & & Marker x Messwerkzeug & 0.91 \\
\cline { 3 - 4 } & & Geschlecht x Marker x Messwerkzeug & \\
& & &
\end{tabular}

Da die Marker einen signifikanten Einfluss auf die nach Kalibrierung errechneten Knochenhöhe hatten, wurden die verschiedenen Marker paarweise gegenüberstellt. Tab. 15 zeigt die Ergebnisse. Hierbei ist zu beachten, dass in dieser Tabelle ein Signifikanzniveau von $\alpha=0.05 / 6=0.0083$ anzunehmen ist.

Tab. 15: $p$-Werte für die paarweisen Vergleiche der nach Kalibrierung bestimmten Knochenhöhendifferenz, getrennt nach Region (Signifikanz ist hier bei $p<0.05 / 6=0.0083$ gegeben)

\begin{tabular}{|c|c|c|}
\hline \multirow[t]{2}{*}{ Vergleich } & \multicolumn{2}{|l|}{ Region } \\
\hline & 46 & 47 \\
\hline Hülse vs. Kugel & 0.5259 & 0.0154 \\
\hline Hülse vs. Plättchen & 0.0009 & 0.7704 \\
\hline Hülse vs. Point & 0.0017 & 0.1195 \\
\hline Kugel vs. Plättchen & 0.0051 & 0.0825 \\
\hline Kugel vs. Point & 0.0085 & 0.0009 \\
\hline Plättchen vs. Point & 0.8819 & 0.1561 \\
\hline
\end{tabular}

Tab. 16 zeigt die Differenz zwischen realer und nach Kalibrierung bestimmter Knochenhöhe. Die stärkste mittlere Abweichung zeigte hier der Hülsenmarker in Regio 46 und der Kugelmarker in beiden Regionen. In diesen Fällen wäre eine Unterschätzung des Knochenangebotes von etwa $0,5 \mathrm{~mm}$ mit einer Standardabweichung von $\pm 0,5 \mathrm{~mm}$ die Folge. Die restlichen ermittelten Knochenhöhen wiesen eine Summe aus Mittelwert und Standardabweichung um $0,5 \mathrm{~mm}$ oder weniger auf. 
Tab. 16: Mittelwert und Standardabweichung der Differenzen zwischen echter und nach Kalibrierung errechneter Knochenhöhe, getrennt nach Markertyp und Region

\begin{tabular}{|c|c|c|}
\hline \multirow[t]{2}{*}{ Marker } & \multicolumn{2}{|l|}{ Region } \\
\hline & 46 & 47 \\
\hline Hülse & $0.53 \pm 0.49$ & $-0.04 \pm 0.31$ \\
\hline Kugel & $0.43 \pm 0.49$ & $0.30 \pm 0.52$ \\
\hline Plättchen & $-0.02 \pm 0.48$ & $0.00 \pm 0.55$ \\
\hline Point & $0.00 \pm 0.50$ & $-0.21 \pm 0.38$ \\
\hline
\end{tabular}

Keinem der Probanden sind die gekippten Röntgenmarker der Hülse, des Plättchens oder des Points im Zuge der Vermessung der Panoramaschichtaufnahmen aufgefallen. Auch bei der Vermessung der Pointmarker an der Röntgenschablone fiel den Probanden eine abweichende Achsstellung nicht auf. 


\subsection{Vergleich OPG XG Plus und NewTom DVT}

Bei dem Vergleich der Panoramaschichtaufnahme mit der digitalen Volumentomographie zeigte sich eine Überschätzung des Knochenangebotes des OPG XG Plus in Regio 46 und 47 und beim NewTom DVT eine Unterschätzung in Regio 46 (Abb. 26). Der Unterschied war signifikant $(p<0.01)$. Die Werte des NewTom DVT in Regio 47 wiesen die niedrigste Differenz zur realen Knochenhöhe auf.

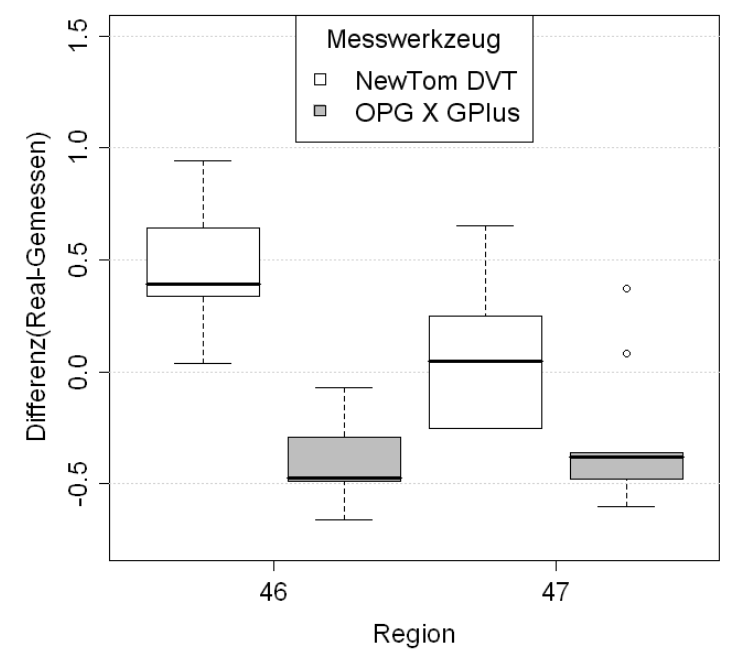

Abb. 26: Verteilungen der Differenzen zwischen realen und gemessenen Knochenhöhen, getrennt nach Region und Messwerkzeug (NewTom DVT und OPG XG Plus) bei $n=10$

Die Mittelwert und Standardabweichungen (Tab. 17) ergaben hierbei eine mittlere Unterschätzung des Knochenangebotes von $0,45 \mathrm{~mm}$ beim NewTom DVT in Regio 46 und eine mittlere Überschätzung des Knochenangebotes von $0,41 \mathrm{~mm}$ beim OPG XG Plus in Regio 46 und $0,31 \mathrm{~mm}$ in Regio 47. Der Mittelwert beim NewTom DVT in Regio 47 wich nur 0,09 mm vom realen Knochenangebot ab. Die Standardabweichungen liegen insgesamt unter $0,31 \mathrm{~mm}$. Die Unterschiede zwischen Regio 46 und 47 sind nicht signifikant $(p=0.15)$.

Der Vergleich der Ergebnisse von DVT und OPG zeigte eine signifikante Wechselwirkung ( $p=0.01$ ), welche in den verschiedenen Regionen unterschiedlich ausfiel. Die Ergebnisse der anschließend durchgeführten paarweisen t-Tests zeigten einen größeren mittleren Unterschied in Region 46. 
Tab. 17: p-Werte, Mittelwert und Standardabweichung der Untersuchung NewTom DVT gegen OPG XG Plus

\begin{tabular}{|l|l|r|r|}
\hline Region & Messwerkzeug & $\begin{array}{l}\text { Mittelwert } \pm \\
\text { Standardabweichung }\end{array}$ & $\mathbf{p}$ \\
\hline 46 & NewTom DVT & $0.45 \pm 0.31$ & $<0.01$ \\
& OPG XG Plus & $-0.41 \pm 0.18$ & $<0.01$ \\
\hline 47 & NewTom DVT & $0.09 \pm 0.30$ & \\
& OPG XG Plus & $-0.312 \pm 0.30$ & \\
\hline
\end{tabular}




\subsection{Zusammenfassung der Ergebnisse}

1. Es konnte ein signifikanter Einfluss des Markertyps bei der Differenz zwischen realer und in den Röntgenaufnahmen gemessener Markerhöhe festgestellt werden. Bei der Gegenüberstellung der Marker zeigte sich die größte Differenz der Markerhöhe beim Pointmarker und die kleinste Markerhöhendifferenz bei der Kugel.

2. Es konnte ein signifikanter Einfluss der Untersuchungsregion bei der Differenz zwischen realer und gemessener Knochenhöhe festgestellt werden. Im Mittel war die Differenz bei Region 46 höher als bei Region 47 (Mittelwert \pm Standardabweichung: 46: $-2.2 \pm 0.4$; 47: $-1.7 \pm 0.4$ ). Bei beiden Regionen kommt es zu einer Überschätzung des vorhandenen Knochenangebotes.

3. Die verschiedenen Messwerkzeuge (Geometriedreieck bzw. digitale Messlehre) hatten keinen signifikanten Einfluss auf das Messergebnis.

4. Die mittleren Vergrößerungsfaktoren fielen mit 1,16 bis 1,21 insgesamt geringer aus als der angegebene 1,25-fache Vergrößerungsfaktors des Programms P1C des verwendeten Panoramaschichtgerätes (Orthophos XG Plus).

5. Das Geschlecht der Probanden hatte keinen signifikanten Einfluss auf das Messergebnis.

6. Nach einfacher Kalibrierung war die Differenz zwischen realer und unter Verwendung der untersuchten Markersysteme gemessener Knochenhöhe geringer als ohne Kalibrierung. Die stärksten mittleren Abweichungen zeigten hier der Hülsenmarker in Regio 46 und der Kugelmarker in beiden Regionen (46 und 47).

7. Der Plättchenmarker zeigte im Mittel die geringste Differenz zwischen realer und nach Kalibrierung gemessener Knochenhöhe. Insgesamt lagen Mittelwert und Standardabweichung der Differenzen zwischen realer und nach Kalibrierung ermittelter Knochenhöhe bei allen Markersystemen innerhalb des geforderten Sicherheitsabstandes von mindestens einem Millimeter. 
8. Beim Regionsvergleich wich die nach Kalibrierung bestimmte Knochenhöhendifferenz nur beim Markertyp Hülse signifikant vom realen Wert ab. In Regio 47 wiesen die Ergebnisse der Berechnung der Knochenhöhe mit Hilfe des leicht gekippten Hülsenmarkers einen geringeren Abstand zur realen Knochenhöhe auf.

9. Beim Vergleich von DVT und OPG zeigten sich eine mittlere Unterschätzung des Knochenangebotes von 0,5 mm beim DVT (NewTom 9000) in Regio 46 und eine mittlere Überschätzung des Knochenangebotes von 0,5 mm beim OPG (Orthophos $X G$ plus) in Regio 46 und 47. Dieser Unterschied war signifikant $(p<0.01)$. Die Unterschiede zwischen Regio 46 und 47 waren dagegen nicht signifikant $(p=0.15)$. 


\section{Diskussion}

\subsection{Motivation zur Untersuchung}

Die Beurteilung des Implantatlagers mittels Panoramaschichtaufnahmen unter Verwendung von Kunststoffschienen mit entsprechenden röntgenopaken Markern definierter Längen gehört zum Standardverfahren in der Implantologie (WEBER 2010). Jedoch gibt es zur Herstellung, Anwendung und möglichen Fehlern nur wenige Untersuchungen (s.u.). Andererseits gibt es zahlreiche Studien zur CT- bzw. DVT-unterstützten, dreidimensionalen präimplantologischen Planung. Diese Verfahren spiegeln aber nicht die alltägliche Praxis wider. Zudem gebietet die Röntgenverordnung (§23) die Anwendung von Diagnoseverfahren mit möglichst geringer Stahlenexposition.

SARMENT et al. (2003) ermittelten Abweichungen von mehr als $2 \mathrm{~mm}$ bei der Verwendung von konventionellen Orientierungsschablonen. Mit Führungsschablonen, die auch intraoperativ eingesetzt wurden, konnten hier Abweichungen vom Implantatapex in vitro von weniger als $1 \mathrm{~mm}$ erreicht werden.

Eine Panoramaschichtaufnahme bietet neben der Diagnostik des Implantatlagers auch eine Übersicht über angrenzende Strukturen und eventuell vorhandene pathologische Befunde wie Osteolysen, Zysten und Tumoren. Eine weiterführende Diagnostik bedarf immer einer besonderen Indikation (Bundesamt für Strahlenschutz 2003, § 23 Röntgenverordnung). So kann eine DVT-Aufnahme bei spezieller Fragestellung ergänzend angefertigt werden. Strahlenhygienische Überlegungen sollten allerdings immer mit im Vordergrund stehen, da die effektive Dosis bei Aufnahmen mit DVT-Geräten um einen Faktor 4-40 über der einer Panoramaschichtaufnahme liegt (S1-Empfehlung der DGZMK zur Dentalen Volumentomographie). Auch bei entsprechender Lückensituation sollte zunächst der prothetische Versorgungswunsch des Patienten geklärt werden, um bei einer röntgendiagnostischen Routineuntersuchung mittels Orthopantommogramm röntgenopake Marker platzieren zu können. 


\subsection{Diskussion der Methodik}

\subsubsection{Das Röntgenmodell}

Durch die Benutzung eines weiterentwickelten Röntgenmodells des Unterkiefers war es in den durchgeführten Untersuchungen möglich, ohne Strahlenbelastung von Probanden verschiedene Markersysteme in Röntgenschablonen direkt miteinander zu vergleichen. Röntgenphantome oder -modelle finden in zahlreichen wissenschaftlichen Studien, in der Dosimetrie oder bei Fragen zur metrischen Genauigkeit neuer Verfahren Anwendung (HASSFELD et al. 1998; WESTENDORFF 2006; PARK et al. 2009; QU et al. 2012). Von Vorteil ist zudem die gute Reproduzierbarkeit der Position des Röntgenmodells, was für die Vergleichbarkeit der erhobenen Messwerte untereinander von entscheidender Bedeutung ist.

\subsubsection{Die Röntgenschablonen}

Bei der kritischen Betrachtung der erstellten Panoramaschichtaufnahmen fallen Details auf, die im Folgenden diskutiert werden sollen.

Die Verschattung durch die Hülse in Regio 46 zeigt eine leichte Kippung zur Senkrechten des Zentralstrahls (siehe Abb. 20). Somit weicht der Marker, der von der Planung aus senkrecht zum Röntgenstrahl stehen sollte, gering ab. Die Hülse in Regio 47 wird dagegen orthoradialer dargestellt.

Der Hülsenmarker in Regio 46, welcher auf den Röntgenaufnahme gekippt erscheint, zeigt durch seine geometrische Form in seiner zweidimensionalen Abbildung Doppellinien am oberen und unteren Rand. Diese könnten durch die Versuchsteilnehmer verschieden gemessen worden sein. Darauf deuten auch die stärkeren Mittelwertabweichungen der Hülse in Regio 46 hin (Abb. 20). Ursache hierfür könnte ein leicht von der Okklusionsebene abweichender Strahlengang sein.

Die Abbildungen der Kugeln sind nicht perfekt rund, sondern leicht hochoval gezeichnet (siehe Abb. 21). Auch die quadratischen Plättchenmarker zeigen in ihrer Abbildung auf der Panoramaschichtaufnahme diesen Effekt (vergleiche Abb. 22). Hier scheint eine bild- bzw. sensornahe Platzierung der betreffenden Marker vorzuliegen.

Dieser Effekt zeigt unmittelbar, dass für die Horizontal- und Vertikaldimension nicht dieselben Vergrößerungsfaktoren benutzt werden können. Zusätzlich variiert der 
Vergrößerungsfaktor in der Horizontalebene (SCHIFF et al. 1986; WELANDER et al. 1987). Dies kann entscheidende Auswirkungen bei der klinischen Auswertung haben, da nicht nur die Länge eines Implantates entscheidend ist. Durch diese aufnahmebedingten Verzerrungen kann der Abstand zu Nachbarzähnen und deren Wurzeln gegebenenfalls nicht sicher geklärt werden.

Die beiden crestal platzierten Marker Point und Plättchen stehen im Röntgenbild nicht genau über den Guttaperchamarkern, welche die Implantationsregionen definieren. So erscheinen die beiden oral platzierten Pointmarker weiter mesial und die beiden vestibulär stehenden Plättchenmarker weiter distal verschoben auf der Panoramaschichtaufnahme. Dies verdeutlichen die vergrößerten Ausschnitte in Abb. 22 und Abb. 23. Diese Beobachtung spricht für einen mesialen Strahlengang. Die crestal platzierten Hülsen- und Kugelmarker stehen dagegen genau über der Implantationsregion.

\subsubsection{Positionierungsfehler}

Trotz sorgfältiger Planung konnte die Röntgengeometrie der Versuchsanordnung nicht absolut perfekt definiert werden.

Das Unterkiefermodell wurde in der Sagittalebene mittig im Panoramaschichtgerät (Orthophos XG Plus) eingebracht. Durch die individuelle Geometrie des Unterkieferkorpus und eine einstellungsbedingte Verschiebung der zu röntgenden Strukturen zur Schicht konnte nicht gänzlich ausgeschlossen werden, dass die Guttaperchamarker, die die reale Knochenhöhe markieren, etwas lingual oder bukkal zur zentralen Röntgenschicht standen.

BEHFAR (2005) beschäftigte sich in einer In-vitro-Studie mit der Fehlposition des Patienten im OPG-Gerät. Fehlpositionierung des Schädels entlang der MedianSagittal-Ebene (X-Achse) und die Rotation des Schädels um die transversale Achse zeigten nur einen relativ geringen Einfluss auf die vertikale Verzerrung der Panoramaschichtaufnahme. Durch Lateralverschiebung des Schädels, Rotation des Schädels um die kranio-kaudale bzw. dorso-ventrale Achse wurden die vertikalen Distanzen dagegen entscheidend beeinflusst. Bei kombinierten Fehleinstellungen traten Summationseffekte auf. Besonders ungünstig war hier die Kombination aus der gleichzeitigen Neigung des Schädels um die dorso-ventrale und die kranio- 
kaudale Achse jeweils zur gleichen Seite. Die verzerrungsbedingten Asymmetrien waren jedoch nicht größer als $4 \%$.

Je nach Versuchsaufbau ergaben sich bei anderen In-vitro-Studien Messabweichungen von im Mittel 2,61 $\mathrm{mm}$ bei der Panoramaschichtaufahme, 0,24 $\mathrm{mm}$ bei der CT-Aufnahme und 0,22 mm bei der DVT-Aufnahme (GOCH 2005). Bei ersterer wurde allerdings ein standardisierter Vergrößerungsfaktor von 1,25 angenommen.

Viele Arbeiten beschäftigten sich mit der Planung mittels dreidimensionaler Bilder. Van ASSCHE et al. (2007) zeigte Abweichungen von bis zu $4^{\circ}$ und lineare Abweichungen von bis zu 2,4 mm. GOCKEL (2009) ermittelte cervical Abweichungen von $1,32 \mathrm{~mm} \pm 0,56 \mathrm{bzw}$. an der Implantatspitze $1,59 \mathrm{~mm} \pm 0,67$ und mittlere Abweichungen der Implantatachsen zwischen geplanter und tatsächlicher Ausrichtung der Implantate von 5,69 $\pm 3,1$. Die CT- und DVT-Daten unterschieden sich hier nicht signifikant.

Aus in vivo Studien liegen zurzeit nur wenige Daten vor, welche sich zudem nur auf geringe Fallzahlen stützen. Hier zeigten sich in Untersuchungen Achsabweichungen von bis zu $11^{\circ}$ und lineare Abweichungen von bis zu $6 \mathrm{~mm}$ (Di GlACOMO et al. 2005). Untersuchungen von MURAT et al. (2012) zur Positionierung von Implantaten ergaben eine mittlere cervicale Abweichung von 1,2 $\pm 0,3 \mathrm{~mm}$ und eine mittlere apikale Abweichung 1,3 $\pm 0,6 \mathrm{~mm}$.

SCHULZE et al. (2000) untersuchten „Auswirkung von Positionierungsfehlern auf Vergrößerungsfaktoren im Unterkiefer bei der digitalen Panoramaschichtaufnahme". Hier zeigte sich, dass vor allem horizontale Positionsänderungen zu ausgeprägten, schwer reproduzierbaren Vergrößerungsfaktoren führen. Insgesamt sei bei der digitalen Schichttechnik mit denselben Verzerrungscharakteristika zu rechnen wie bei konventionellen Aufnahmen. 


\subsubsection{Genauigkeit der radiologischen Abbildungen}

Sowohl bei der präoperativen Planung mittels Panoramaschichtaufnahme als auch mit Hilfe der digitalen Volumentomographie können sich technik- und anwenderbedingt eine Reihe von möglichen Fehlerquellen ergeben, welche zu Abweichungen vom realen Implantatbett führen können.

Tab. 18: Mögliche Fehlerquellen bei Orthopantomographie und digitaler Volumentomographie

\begin{tabular}{|c|c|}
\hline Orthopantomographie & digitale Volumentomographie \\
\hline \multicolumn{2}{|c|}{ In der Bilderfassung: } \\
\hline $\begin{array}{l}\text { - Schichtdicke und -verlauf } \\
\text { - Auflösungsvolumen des Sensors } \\
\text { - Patientenpositionierung } \\
\text { - Positionierung der Rö-Marker } \\
\text { - Bewegungsartefakte, } \\
\text { (Patientenbewegung) }\end{array}$ & $\begin{array}{l}\text { - Schichtdicke } \\
\text { - Voxelgröße } \\
\text { - Metallartefakte } \\
\text { - Bewegungsartefakte, } \\
\text { (Patientenbewegung) }\end{array}$ \\
\hline \multicolumn{2}{|c|}{ Bei der Auswertung: } \\
\hline \begin{tabular}{|l|} 
- Summationseffekte \\
- Verzeichnung und Vergrößerung \\
- Interpretation und Messung
\end{tabular} & $\begin{array}{l}\text { - Rekonstruktion } \\
\text { - Schichtauswahl } \\
\text { - Interpretation und Messung }\end{array}$ \\
\hline
\end{tabular}

Mehrere Studien beschäftigen sich mit dem Vergleich der Präzision verschiedener radiologischer Diagnoseverfahren in der Zahnmedizin (FOET 2007, HÜBINGER 2008, KLINGBERG 2008, CONRAD 2010). Hier zeigen die dreidimensionalen bildgebenden Verfahren wie CT und DVT in der Regel eine geringfügig höhere metrische Genauigkeit als Panoramaschichtaufnahmen.

Das hier verwendete Panoramaschichtgerät Orthophos XG Plus (Sirona) erreicht mit seinen CCD-Sensoren eine Pixelgröße von $27 \mu \mathrm{m}$ in der 2D-Darstellung. Dagegen verfügen digitale Volumentomographen wie das NewTom 9000 über eine Voxelgröße von $250 \mu \mathrm{m} \times 250 \mu \mathrm{m} \times 200 \mu \mathrm{m}$. Eine höhere mögliche Auflösung, aber ein kleineres abgebildetes Volumen, erreicht der digitale Volumentomograph Accuitomo (Fa. Morita, Kyoto, Japan) mit einer Voxelgröße von $80 \mu \mathrm{m}$. Neuste Geräte erreichen eine Voxelgröße von $50 \mu \mathrm{m}$ (Planmeca). Die Auflösung wird allerdings durch die Patientenbewegung limitiert, da eine Aufnahme je nach Gerät 20-72 s dauert 
(KYRIAKOU et al. 2011). Metaanalysen zur effektiven Dosis von dentalen Volumentomographen zeigen eine sehr große Bandbreite der effektiven Dosen von $5 \mu \mathrm{Sv}$ bis $1073 \mu$ Sv (LUDLOW et al. 2015). Geringere effektive Dosen werden zulasten des erfassten Volumens oder der Ortsauflösung erreicht.

\subsection{Diskussion der Ergebnisse}

Die Aufgabenstellung, mit beiden Messwerkzeugen möglichst genau die Markerlänge und das Knochenangebot auf den Panoramaschichtaufnahmen zu bestimmen, wurde von den Probanden sehr genau umgesetzt. Dies verdeutlichen insbesondere die einzelnen Messwerte, die mit Hilfe des Geometriedreiecks erhoben wurden. Die Auswahl der im ersten Versuch zur Verfügung gestellten Messwerkzeuge Geometriedreieck und elektrische Schieblehre, hatten keinen signifikanten Einfluss auf das Ergebnis der Messung. Auch ein Einfluss des Geschlechtes der Probanden auf die Messungen war nicht feststellbar.

\subsubsection{Vergleich verschiedener Markersysteme im OPG XG Plus}

Auf Grundlage der Überlegungen zur Röntgengeometrie ergeben sich folgende allgemeine klinische Vor- und Nachteile der untersuchten Markersysteme.

Der Hülsenmarker erlaubt eine Beurteilung des vertikalen und auch des horizontalen Knochenangebotes. Hierbei ist auf die Überlagerung der Abbildung der Ränder des zylinderartigen Markerkörpers zu achten, wenn dieser nicht genau senkrecht zum Zentralstahl ausgerichtet ist. Dieser Marker ist zudem für den intraoperativen Einsatz vorgesehen.

Der besondere Vorteil des Kugelmarkers ist die radialsymmetrische Form, durch die der Marker kippungsunabhänig bei der Ausrichtung zur Röntgenanlage ist. Nachteil ist die ausschließliche Verwendung als Messschablone, da sich die Kugeln genau kranial der Implantationsregion befinden. Somit ist die nachfolgende Verwendung als intraoperative Bohrschablone nicht möglich.

Vorteile des Plättchenmarkers sind die gute Beurteilbarkeit des vertikalen und des horizontalen Knochenangebotes durch separat beurteilbare Vergrößerungsfaktoren. Dabei ist jedoch zu beachten, dass der Marker kippungsabhänig ist. Somit muss 
dieser senkrecht zum Zentralstahl ausgerichtet werden, um reproduzierbare Ergebnisse zu erlangen. Seine vestibuläre Lage zum Implantatlager bedeutet eine film- beziehungsweise sensornähere und dadurch verkleinerte Abbildung. Diese hat nach den vorliegenden Ergebnissen aber keine klinische Bedeutung. Zudem ist der Marker dadurch auch bei Schablonen, die durch Führungslöcher intraoperativ Verwendung finden, geeignet.

Vorteile des Pointmarkers sind die individuell Längenanpassung an vertikale Platzverhältnisse und seine günstigen Kosten. Andererseits erlaubt er lediglich eine Beurteilung des vertikalen Knochenangebotes. Hierbei muss beim Kalibriervorgang zudem in jedem Fall der Marker jeder Implantationsregion separat gemessen werden, da dieser nicht wie die anderen untersuchten Marker längenkalibriert ist. Außerdem ist zu beachten, dass der Marker kippungsabhänig ist. Seine orale Lage zum Implantatlager bedeutet eine film- beziehungsweise sensorfernere und dadurch theoretisch vergrößerte Abbildung, die aber nach den vorliegenden Ergebnissen klinisch keine Bedeutung hat. Zudem kann der Marker auch bei Schablonen eingesetzt werden, die intraoperativ Verwendung finden.

Es zeigte sich eine Abhängigkeit vom Markertyp und der Region bei der Differenz der realen Markerlänge zu der durch die Versuchsteilnehmer ermittelten Länge. Zudem konnte ein signifikanter Einfluss der Region bei der Differenz zwischen realer und gemessener Knochenhöhe festgestellt werden. Diese Ergebnisse können wie folgt eingeordnet werden.

Für das Erstellen der Orthopantomogramme wurde das Programm P1 C mit einer konstanten 1,25-fachen Vergrößerung gewählt. Daher musste mit einer entsprechenden Vergrößerung der Abbildung der Röntgenmarker auf der Panoramaschichtaufnahme gerechnet werden. Die Missachtung dieses Vergrößerungsfaktors führt zu einer Überschätzung des Knochenangebotes und damit intraoperativ zu möglichen Verletzungen der zu schützenden Strukturen wie Kiefer- und Nasenhöhlenboden oder Nervus alveolaris inferior.

Die Abweichungen der Mittelwerte für die Differenz der gemessenen Markerlänge zu der realen Markerlänge (siehe Ergebnisse Abb. 20, Abb. 21, Abb. 22 und Abb. 23) korrelierten in etwa wieder mit dem durchschnittlichen Vergrößerungsfaktor durch das Programm P1 C der Panoramaaufnahme von 1,25 und konnten sowohl für Regio 
46 als auch für Regio 47 durch die verschiedene Länge der Röntgenmarker erklärt werden.

Von den verwendeten Röntgenmarkern hatten der Hülsen- und der Pointmarker in der Vertikalen die größte metrische Ausdehnung und zeigten daher bei einer Kippung zur Röntgenebene höhere messbare Abweichungen.

Die Ergebnisse der Messungen zeigten allerdings, dass sowohl in Regio 46 als auch 47 die Abweichung der gemessenen Markerlängen durch den aufnahmebedingten Vergrößerungsfaktor geringer ausfielen, als es die 1,25-fache Vergrößerung des Programms P1 C vermuten lassen würde (siehe Ergebnisse Abb. 20, Abb. 21, Abb. 22, Abb. 23 und Tab. 9).

Auch bei der direkten Messung des Knochenangebotes zeigen sich Differenzen zwischen realer und gemessener Knochenhöhe. Hier würde es zu einer deutlichen Überschreitung des Sicherheitsabstandes zum Mandibularkanal und somit zu einer möglichen Nervschädigung kommen.

Dies verdeutlicht die Wichtigkeit der Verwendung von röntgenopaken Markern definierter Längen und die Berücksichtigung des hiermit ermittelten Vergrößerungsfaktors, da die gerätetypischen Vergrößerungen zum Teil stark schwanken (SCHULZE et al. 2000; ROMÁN et al. 2003).

Nach der Kalibrierung ergaben sich insgesamt geringere Abweichungen zur realen Knochenhöhe. Der Hülsen- und der Kugelmarker führten im Mittel zu einer Unterschätzung von $0,5 \mathrm{~mm}$. Plättchen- und Pointmarker zeigten bei den ungekippten Markern in Regio $46 \mathrm{im}$ Mittel noch geringere Abweichungen. Bei den Markern in Regio 47 erzielte der Hülsenmarker die besten Ergebnisse (siehe Ergebnisse Abb. 25). Insgesamt streuten die Werte weniger als $2 \mathrm{~mm}$ um den jeweiligen Median.

Die stärksten Abweichungen fanden sich beim Hülsenmarker in Regio 46 und beim Kugelmarker in beiden Regionen. Nach den erhobenen Messwerten kam es hier zu einer Unterschätzung des Knochenangebotes.

Die Abweichung des radialsymmetrischen Kugelmarkers lässt den Schluss zu, dass die Kugelmarker nicht ganz genau in der Schicht standen, bei der der programmierte Vergrößerungsfaktor 1,25 erreicht wird. Eine mögliche Ursache wäre eine sensorferne Lage des Kugelmarkers, die zu einer vergrößerten Abbildung führen 
würde. So könnte nach Kalibrierung eine Unterschätzung des Knochenangebotes resultieren.

Beim Markertyp „Hülse“ zeigte der Regionsvergleich eine unterschiedliche Knochenhöhendifferenz. So ließen die Messungen mit dem Hülsenmarker in Regio 46 im Mittel auf ein geringeres Knochenangebot schließen. Hier könnte ebenfalls die vestibuloorale Lage des Markers Einfluss gehabt haben. Weiterhin wäre es möglich, dass der nicht axial geröntgte Hülsenmarker in Regio $46 \mathrm{zu}$ einer vergrößerten Abbildung auf dem Sensor führte (siehe Abb. 20 und Abb. 10). Bei genauer Betrachtung zeigte die Verschattung der Hülse in dieser Region eine Kippung zur Senkrechten des Zentralstrahls. Somit wich der Marker, der von der Planung aus senkrecht zum Röntgenstrahl stehen sollte, gering ab.

Die Verschattung der Hülse in Regio 47 dagegen schien orthoradialer zu stehen. Hier führte die Summe der Einflussfaktoren im Median zu einer genaueren Bestimmung der realen Knochenhöhe.

Den Probanden sind im Röntgenbild gekippt abgebildete Marker nicht aufgefallen. Die Suche nach gekippten Objekten durch die Versuchsteilnehmer war aber auch nicht explizit Aufgabe bei der Messung der röntgenologischen Markerlänge und des röntgenologischen Knochenangebotes auf den Panoramaschichtaufnahmen. Eine gezielte Frage danach hätte möglicherweise von der eigentlichen Aufgabe abgelenkt. 


\subsubsection{Vergleich OPG XG Plus und NewTom DVT}

Beim Vergleich von OPG XG Plus und NewTom DVT zeigte sich ein signifikanter Unterschied. Die Daten, die mit dem NewTom DVT erhoben wurden, zeigten eine Unterschätzung des Knochenangebotes. Die Auswertung des Orthophos XG Plus dagegen zeigte eine Überschätzung des Knochenangebotes. Beide mittleren Abweichungen lagen im Bereich von weniger als $1 \mathrm{~mm}$ vom realen Knochenangebot und somit im Bereich des empfohlenen Sicherheitsabstandes. Dabei wichen die Werte im Mittel nicht mehr als $0,5 \mathrm{~mm}$ ab und hatten eine geringe Streuweite von $0,3 \mathrm{~mm}$.

Die Werte der metrischen Genauigkeit der Untersuchungen mit dem DVT waren mit denen anderer Untersuchungen mit ähnlichen Fragestellungen zu vergleichen. GOCH (2005) ermittelte Messabweichungen von im Mittel 2,61 mm bei der Panoramaschichtaufahme wobei ein gerätespezifischer standardisierter Vergrößerungsfaktor von 1,25 angenommen wurde. Die metrische Genauigkeit der CTAufnahme lag hier bei 0,24 mm und mit der DVT-Aufnahme bei 0,22 mm. KÜKER (2010) verglich die metrische Genauigkeit von CT, DVT und konventionellen Röntgentechniken und erreichte vor allem bei gekippten Objekten eine höhere metrische Genauigkeit bei den dreidimensionalen bildgebenden Verfahren. CONRAD (2010) ermittelte durchschnittliche vertikale Vergrößerungsfaktoren von 1,00 beim NewTom QR-DVT 9000 und bei den Panoramaschichtgeräten geräteabhängig zwischen 1,05 und 1,25.

Die Unterschätzung des Knochenangebotes bei der Untersuchung mittels DVT könnte auf die im Vergleich zum Orthophos XG Plus geringere Auflösung der rekonstuierten Datensätze des NewTom DVT zurückgeführt werden. Die geringere Voxelgröße und die berechneten Schnittbilder von 0,3 mm lagen deutlich unter der Auflösung der digitalen Panoramaschichtaufnahme.

Darauf deutete auch der geringere Interquartilsabstand bei Verwendung des Orthophos OPG XG Plus hin. Dieser war deutlich geringer als bei der Verwendung des NewTom DVT. Auch die höhere Auflösung des Bildes und die Möglichkeit der größeren Darstellung der zu vermessenden Region auf der Arbeitsoberfläche beim Programm des Orthophos XG Plus „SIDEXIS neXt Generation 2.4“ könnten hierfür ursächlich sein. Dagegen war die Vermessung der Datensätze mit dem im Programm „NEWTOM; QR-DVT 9000-study“ des NewTom DVT integrierten Messwerkzeuges 
durch eine geringere maximale Vergrößerung des relevanten Bildausschnitts erschwert.

Tab. 19 stellt die Vor- und Nachteile der digitalen Volumentomographie gegenüber der Orthopantomographie in der präimplantologischen Planung unter Bezug auf die Ergebnisse der vorliegenden Arbeit dar.

Tab. 19: Vor- und Nachteile von digitalem OPG vs. DVT bei der präimplantologischen Diagnostik

\begin{tabular}{|c|c|}
\hline OPG (Orthophos XG Plus) & DVT (NewTom 9000) \\
\hline $\begin{array}{l}\text { Hohe optische Auflösung der zur } \\
\text { Vermessung verfügbaren Bilddaten }\end{array}$ & $\begin{array}{l}\text { Relativ niedrige optische Auflösung } \\
\text { (kann erhöht werden wenn eine höhere } \\
\text { Strahlendosis akzeptiert wird) }\end{array}$ \\
\hline $\begin{array}{l}\text { Digitales Messwerkzeug muss vor der } \\
\text { Messung kalibriert werden }\end{array}$ & Keine Kalibrierung erforderlich \\
\hline $\begin{array}{l}\text { Einstellung des Patienten mit } \\
\text { Positionierung des Markers und Verlauf } \\
\text { zum Rö-Strahl beeinflussen stark das } \\
\text { Ergebnis }\end{array}$ & Weniger einstellungssensibel \\
\hline Bewegungsartefakte & $\begin{array}{l}\text { Hohe Anfälligkeit für } \\
\text { Bewegungsartefakte }\end{array}$ \\
\hline $\begin{array}{l}\text { Geringe Artefakte durch metallene } \\
\text { Strukturen- Darstellung periimplantärer } \\
\text { Knochenstrukturen }\end{array}$ & Metallartefakte \\
\hline Zweidimensionale Abbildung & $\begin{array}{l}\text { Durch transversale Schnittbilder kann } \\
\text { auch bei schwierigen Fragestellungen } \\
\text { das Knochenlager dargestellt werden }\end{array}$ \\
\hline
\end{tabular}

In dem mit dem DVT durchgeführten Versuch hatte die Region beim verwendeten Kugelmarker keinen signifikanten Einfluss. Die Ausdehnung der Abbildung der beiden Kugelmarker war identisch. Der Kugelmarker wurde in beiden Regionen jeweils crestal des zu vermessenden Knochenabschnitts angebracht und zeigte durch seine geometrische Form in jeder Betrachtungsebene dieselbe Ausdehnung. Hier ist davon auszugehen, dass die beiden Kugeln in vestibulooraler Richtung gleich in der Schicht stehen. 
Die vertikalen Ausmessungen der Kugeln wurden zur Kalibrierung der Software SIDEXIS neXt Generation 2.4 des Orthophos XG Plus eingesetzt. Die Knochenangebote der beiden Implantationsregionen konnten so anschließend durch die Versuchsteilnehmer direkt über das in der Software integrierte Messwerkzeug bestimmt werden.

Die Software QR-DVT 9000-study des NewTom 9000 ermöglichte eine direkte Vermessung des Implantatlagers, ohne dass zuvor eine Kalibrierung stattfinden musste. Für die Vermessung gaben die Kugeln der Markerschablone lediglich die Implantationsposition vor. Somit war die Abweichung vom realen Knochenangebot der beiden Regionen sehr gering.

Die Entwicklung von CT/DVT-gestützten implantatprothetischen Führungsschablonen hat auch intraoperativ zu einer höheren Präzision bei der Implantation geführt (NICKENIG und SPIEKERMANN 2006). Auch Untersuchungen von WESTENDORFF (2006) zur computergestützten Planung und bilddatengestützten Navigation unter Zuhilfenahme eines CT-Daten gestützten Navigationssystems zeigen einen Trend zu immer präziseren, aber auch techniksensibleren Implantationsverfahren.

Die Differenz zwischen realer und gemessener Knochenhöhe zeigte einen Einfluss des Messwerkzeuges (hier Gesamtsystem der Kugelmarkerschablone im OPG XG Plus und der Kugelmarkerschablone im NewTom DVT). Hier war der mittlere Unterschied in Regio 46 etwas größer. Eine mögliche Erklärung für diesen Unterschied könnte im Versuchsaufbau und der Breite der rekonstruierten Schichten mit dem NewTom DVT begründet liegen. Das Schnittbild, welches den Versuchsteilnehmern zur Vermessung vorgelegt wurde, zeigte die vier Guttaperchamarker, die das Knochenangebot definierten. Durch die vorgegebene Schichtstärke der rekonstruierten Bilder von $0,3 \mathrm{~mm}$ und der erstellten Schnittbilder durch den Kieferkamm von 0,5 mm kann nicht zwingend davon ausgegangen werden, dass alle vier Guttaperchamarker je mit ihrer größten Ausdehnung getroffen wurden. 


\subsection{Schlussfolgerungen für die klinische Anwendung}

Die individuelle Form des Zahnbogens und bukkolinguale Neigung des Corpus mandibulae im Seitenzahngebiet erfordern eine möglichst genaue Beurteilung des Knochenlagers im Rahmen der präimplantologischen Voruntersuchungen. Klinische Untersuchung und bildgebende Diagnostik gehören dabei eng zusammen. In manchen Fällen kann zusätzlich eine dreidimensionale Röntgenaufnahme indiziert sein, um eine ausreichende Planungssicherheit zu gewährleisten.

Die durchgeführten Untersuchungen konzentrierten sich auf das vertikale Knochenangebot. Zu beachten war, dass das Implantatlager stets eine dreidimensionale Ausdehnung hat. Die Angulation des erforderlichen Implantats oder dessen Position wurden durch den Versuchsaufbau fest definiert.

Sowohl bei analog als auch bei digital erstellte Panoramaschichtaufnahmen zeigen sich immer technikbedingt vergrößerte Abbildungen der geröntgten Strukturen. Die Sensortechnik und die Verarbeitung der Bilddaten über eine Computerschnittstelle beim hier verwendeten Orthophos XG Plus erlaubt zwar eine Einstellung der Druckgröße von $100 \%$, welche aber nicht als Wiedergabe der realen Ausdehnung der abgebildeten anatomischen Strukturen interpretiert werden darf. Die Ergebnisse zeigen, dass eine einfache Messung der Knochenhöhe am Röntgenbild zur Bestimmung des realen Knochenangebotes nicht ausreichend genau ist. Daher sollten entsprechende Planungsaufnahmen immer mit einem Röntgenmarker als Referenz erstellt und erst nach Kalibrierung metrisch ausgewertet werden.

Die Vergrößerungsfaktoren können zusätzlich innerhalb der Horizontal- oder Vertikaldimension variieren (SCHULZE et al. 2000). Das zeigen auch die Untersuchungen der Abbildungen der verschiedenen Röntgenmarker in der vorliegenden Arbeit. Die nicht crestal platzierten Plättchen- und Pointmarker weichen in mesiodistaler Richtung von der vorgesehenen Implantatregion ab.

Implantatumrissschablonen mit standardisierten Vergrößerungsfaktoren sind nur bedingt einsetzbar, da vertikale und horizontale Vergrößerungsfaktoren innerhalb eines Orthopantomogramms stark schwanken können (SCHULZE et al. 2000). Diese sollten daher lediglich einen orientierenden Charakter haben. Die unmittelbare Messung der Knochenhöhe am Röntgenbild zur Bestimmung des realen Knochenangebotes ist nicht ausreichend genau und daher obsolet. Eine Referenzierungsschablone ist stets zu fordern. 
Die Point- und Plättchenmarker können durch ihre orale beziehungsweise vestibuläre Stellung zum Implantatlager zu fehlerhaften Projektionen in mesiodistaler Richtung führen. Beim Hülsenmarker und beim Kugelmarker kann dieses Problem durch ihre crestale Stellung weitestgehend ausgeschlossen werden.

$\mathrm{Da}$ die Bewegung moderner Panoramaschichtgeräte während des Röntgenvorganges komplex ist und Verzerrungen innerhalb der Panoramaschichtaufnahme unvermeidbar sind, sollte stets für jede Implantationsstelle ein Marker in der präimplantologischen Diagnostik vorgesehen werden. Der daraus ermittelbare jeweilige Vergrößerungsfaktor ist die grundlegende Voraussetzung für eine präzise Bestimmung des vorhandenen Knochenangebotes. Die Marker sollten immer möglichst senkrecht zum Sensor ausgerichtet werden, da Projektionsfehler durch Kippung der Marker oder des Knochenlagers zur Schicht nach Erstellen der Aufnahmen nur noch schwer erkannt oder nachvollzogen werden können.

Die Verwendung der Vermessungssoftware des OPG (Orthophos XG Plus) zusammen mit Kalibrierung durch den Kugelmarker ermöglichte einen geringeren Interquartilsabstand der Untersuchungsergebnisse. So können Fehler bei der manuellen Vermessung sowie bei der Umrechnung des Vergrößerungsfaktors vermieden werden.

Die Messschablone des Kugelmarkersystems kann allerdings nicht gleichzeitig als Operationsschablone genutzt werden. Die Systeme des Point-, Titanhülsen- und Plättchenmarkers können dagegen intraoperativ zur Körnung und zur Pilotbohrung genutzt werden. Der Operateur erhält so wichtige Informationen über die prothetische Implantatposition und Achsrichtung.

Eine Kombination mehrerer Markersysteme in einer Messaufnahme/Messschablone könnte zu einer höheren Planungssicherheit beitragen. Ein Beispiel wäre die Verwendung des Kugelmarkers zum Bestimmen des standardisierten Vergrößerungsfaktors und von Hülsen zum Markieren der einzelnen Implantationsregionen und als Führung der Angulation für den Pilotbohrer.

Wichtig ist hier das Zusammenspiel von Zahnarzt und Zahntechniker. Der Zahntechniker ist beim Erstellen der Referenzierungs- oder Operationsschablonen eher prothetisch orientiert. Der Operateur muss zusätzlich die zu schützenden anatomischen Strukturen und die Morphologie und Physiologie des Knochenlagers 
berücksichtigen. Daher ist ein enger Informationsaustausch zum Erreichen des individuellen Optimums für den Patienten unabdingbar.

Die leicht gekippte Stellung der Röntgenmarker in Regio 47 hatte in den durchgeführten Untersuchungen nur beim Hülsenmarker einen signifikanten Einfluss auf das ermittelte Knochenangebot (Regio 46: $0.53 \pm 0.49$; Regio 47: $-0.04 \pm 0.31$ ). Eine leichte Lingualkippung der Implantatachse ist in manchen Fällen aus prothetischer Sicht geboten. Daher ist es möglich, Marker wie die Bohrhülsen, die gleichzeitig als Richtungsvorgabe für die Implantatachse dienen, im gewissen Rahmen der prothetischen Achse anzugleichen.

Die metrische Genauigkeit der erzielten Ergebnisse zum Errechnen des vertikalen Knochenangebotes über die Auswertung von Panoramaschichtaufnahmen stimmt im Wesentlichen mit den Ergebnissen der digitalen Volumentomographie überein. Entscheidend hierfür könnten die exakte geometrische Planung der Versuchsanordnung und des Röntgenmodells sein.

Sowohl die Qualität computergestützter Planungen mittels dreidimensionaler Bilddaten als auch deren Anzahl haben durch die rasante technische Entwicklung in den letzten Jahren stark zugenommen (Bundesamt für Strahlenschutz 2012). Hierbei müssen stahlenprophylaktische und wirtschaftliche Aspekte der Planungssicherheit gegenüber abgewogen werden.

In den vorliegenden Untersuchungen konnte beim Vergleich von Panoramaschichtaufnahme und digitaler Volumentomographie ein signifikanter Unterschied festgestellt werden. Dieser Effekt kann aber nach den vorliegenden Ergebnissen als klinisch weniger relevant eingestuft werden, da sich die Abweichungen der DVTMessungen nicht maßgeblich von denen der Auswertung der Panoramaschichtaufnahmen unterscheiden.

Unter Verwendung der Panoramaschichttechnik gelang es allen Versuchsteilnehmern, in den vorliegenden Untersuchungen die Knochenhöhe präoperativ innerhalb des allgemein geforderten Sicherheitsabstandes von 1-2 mm zu bestimmen. Die Vermessung des DVT-Datensatzes durch die Versuchsteilnehmer erbrachte etwas bessere Mittelwert und Standardabweichungen, die nur bis zu $0,7 \mathrm{~mm}$ vom eigentlichen Knochenangebot abwichen.

Die Ergebnisse dieser Arbeit können auch auf den Oberkiefer übertragen werden, da sich hier eine ähnliche Problematik ergibt. Die Lage des Kieferhöhlenbodens im Implantationsgebiet kann zweidimensional nur schwer beurteilt werden. Zudem ist 
gerade im distalen Oberkieferseitenzahnbereich das Knochenangebot durch die schnelle Atrophie nach Zahnverlust in vielen Fällen eingeschränkt.

Sowohl die vorliegenden Ergebnisse als auch die diskutierten Studien belegen, dass trotz genauer Planung immer eine gewisse Streubreite der Messwerte und somit der räumlichen Abweichung vom geplanten Implantatlager besteht. Daher sollte bei der Planung der Insertionstiefe im Bereich des N. alveolaris inferior in jedem Fall der geforderte Sicherheitsabstand von 1-2 mm eingehalten werden, da sonst die Gefahr einer iatrogenen Nervschädigung besteht.

Abschließend lässt sich sagen, dass die Panoramaschichtaufnahme mit einer Referenzierungsschablone mit den untersuchten Markersystemen weiterhin für die meisten Fälle der implantologischen Basisdiagnostik geeignet ist, wobei der Plättchenmarker im Mittel die genauste Bestimmung der Knochenhöhe ermöglichte. Klinisch muss aber fallbezogen beurteilt werden, ob bei kritischer Knochenhöhe und möglicher Gefährdung anatomisch wichtiger Strukturen zusätzlich eine dreidimensionale Bildgebung notwendig ist. 


\section{Zusammenfassung}

Die Panoramaschichtaufnahme unter Verwendung einer Röntgenschablone mit Referenzierungsmarkern wird als Basisdiagnostik der präimplantologischen Planung angesehen. Während es zur dreidimensionalen präimplantologischen Planung mit Schablonen zahlreiche Studien gibt, fehlen systematische Untersuchungen zur Eignung unterschiedlicher Markersysteme für die Panoramaschichtaufnahme.

Ziel der Arbeit war der direkte Vergleich verschiedener Markersysteme wie Hülsen, Kugeln, Plättchen oder Guttaperchapoints bei Aufnahmen mit dem Panoramaschichtgerät. Dazu wurde ein selbstständig weiterentwickeltes Röntgenmodell eines teilbezahnten Unterkiefers verwendet. Für jedes Markersystem wurde eine Planungsschalone mit Markern für Implantate in Regio 46 und 47 angefertigt und mit einem digitalen Panoramaschichtgerät geröntgt. Hierbei stand der Marker in Regio 47 leicht zur Okklusionsebene gekippt.

Die Auswertung der Aufnahmen durch Probanden wurde nach Rücksprache mit dem Institut für Medizinische Statistik der Universitätsmedizin Göttingen geplant. Die vier Aufnahmen wurden von je 10 Versuchsteilnehmern ausgewertet. Des Weiteren wurde vom Röntgenmodell eine Aufnahme mit dem digitalen Volumentomographen NewTom 9000 angefertigt. Diese wurde durch 10 Versuchsteilnehmer vermessen und die Ergebnisse mit denen der Panoramaschichtaufnahme verglichen. Die verschiedenen Einflussfaktoren wie Messinstrument, Geschlecht, Region und abweichende Stellung der Marker wurden mit mehrfaktoriellen Varianzanalysen inklusive aller Wechselwirkungen untersucht.

Die Ergebnisse zeigten einen signifikanten Einfluss der Region bei der Differenz zwischen realer und gemessener Knochenhöhe. Im Mittel war die Differenz bei Region 46 höher als bei Region 47 (Mittelwert \pm Standardabweichung: Regio 46: -2.2 \pm 0.4; Regio 47: $-1.7 \pm 0.4$ ). Die mittleren Vergrößerungsfaktoren waren bei allen Markern geringfügig niedriger $(1,16-1,21)$ als der für das Gerät ausgewiesene Vergrößerungsfaktor von 1,25. Das verwendete Messwerkzeug oder das Geschlecht der Probanden hatten keinen signifikanten Einfluss auf das Messergebnis.

Nach Kalibrierung lagen Mittelwert und Standardabweichungen insgesamt bei allen Markersystemen innerhalb des geforderten Sicherheitsabstandes von mindestens einem Millimeter zum Nervkanal. Beim Regionsvergleich war die nach dem Kalibrier- 
vorgang bestimmte Differenz zwischen realer und gemessener Knochenhöhe nur beim Markertyp Hülse signifikant. Die Berechnung der Knochenhöhe mit Hilfe des leicht gekippten Hülsenmarkers in Regio 47 lag hier näher an der Höhe des realen Knochenangebotes. Der Plättchenmarker zeigte im Mittel die geringste Differenz zwischen realer und nach Kalibrierung gemessener Knochenhöhe.

Beim Vergleich der Panoramaschichtaufnahme mit der digitalen Volumentomographie zeigte sich ein signifikanter Unterschied $(p<0.01)$. Es kam zu einer mittleren Unterschätzung des Knochenangebotes von $0,5 \mathrm{~mm}$ beim DVT (NewTom 9000) in Regio 46 und einer mittleren Überschätzung des Knochenangebotes von $0,5 \mathrm{~mm}$ beim Panoramaschichtgerät (Orthophos XG Plus) in Regio 46 und 47. Der Regionseffekt war dagegen nicht signifikant $(p=0.15)$.

Als Schlussfolgerung für die klinische Anwendung kann die Panoramaschichtaufnahme unter Verwendung einer Referenzierungsschablone für die präimplantologische Röntgendiagnostik bei Fällen ohne besondere Schwierigkeit als hinreichend genau betrachtet werden. Unter dem Gesichtspunkt der Strahlenhygiene sollte die digitalen Volumentomographie komplexen Fällen vorbehalten werden, da dem zusätzlichen Informationsgewinn durch dieses Verfahren eine im Vergleich zur Panoramaschichtaufnahme deutlich erhöhte Strahlenexposition gegenübersteht. 


\section{Literaturverzeichnis}

Augthun M, Munt T (2008): Implantatprothetische Konzepte zur Ergänzung der verkürzten Zahnreihe. Gemeinsame Stellungnahme der Deutschen Gesellschaft für Zahnärztliche Prothetik und Werkstoffkunde (DGZPW) und der Deutschen Gesellschaft für Zahn-, Mund- und Kieferheilkunde (DGZMK). [Zugriff am 9.4.2013]. URL: http://www.dgzmk.de/uploads/tx_szdgzmkdocuments/Implantatprothetische_Konzepte_zur_Ergaenzung_der_verkuerzten_Zahnreihe_.pdf

Behfar L: Fehlpositionierungsbedingte „Verzerrungen“ der Panoramaschichtaufnahme. Med. Dent. Diss. Hamburg 2005

Beyer D, Herzog M, Zanella F, Bohndorf K, Walter E, Hüls A: Röntgendiagnostik von Zahn- und Kiefererkrankungen. Ein klinisch-radiologisches Konzept; Springer Verlag, Berlin 2011

Bundesamt für Strahlenschutz: Umweltradioaktivität und Strahlenbelastung im Jahr 2012: Unterrichtung durch die Bundesregierung. Vom 3.4.2014 [Zugriff am 30.4.2015]. URL: http://nbn-resolving.de/urn:nbn:de:0221-2014040311384

Bundesministerium für Umwelt, Naturschutz, Bau und Reaktorsicherheit: § 2c: Vermeidung unnötiger Strahlenexposition und Dosisreduzierung. In:

Bekanntmachung der Neufassung der Röntgenverordnung. Vom 30. April 2003. Bundesgesetzblatt Jahrgang 2003 Teil I Nr. 17, Bonn 2003 (zuletzt geändert durch Art. 6 V v. 11.12.2014/2010) [Zugriff am 9.5.2015]. URL: http://www.gesetze-iminternet.de/bundesrecht/r_v_1987/gesamt.pdf

Buser D, Mericske-Stern R, Bernard JP, Behneke A, Behneke N, Hirt HP, Belser UC, Lang NP (1997): Long-term evaluation of non-submerged ITI implants. Part 1: 8-year life table analysis of a prospective multi-center study with 2359 implants. Clin Oral Implants Res $\underline{8}(3), 161-172$ 
Cacaci C, Neugebauer J, Schlegel A, Seidel F: Orale Implantologie; Georg Thieme Verlag, Stuttgart 2006

Conrad C: Vergleich der metrischen Genauigkeit von extraoralen Röntgenverfahren:

Digitale Volumentomographie versus konventionelle und digitale

Orthopantomographie- In-vitro-Studie an humanen Schädelpräparaten. Med. Dent.

Diss. Gießen 2010

Di Giacomo GA, Cury PR, de Araujo NS, Sendyk WR, Sendyk CL (2005): Clinical application of stereolithographic surgical guides for implant placement: preliminary results. J Periodontol $\underline{76}(4), 503-507$

Foet JH: Vergleich verschiedener diagnostischer Verfahren zur Schonung des Nervus Alveolaris inferior bei der enossalen Implantation im Unterkieferseitenzahngebiet. Med. Dent. Diss. Bonn 2007

Frasch G, Kammerer L, Karofsky R, Mordek E, Schlosser A, Spiesl J: Die berufliche Strahlenexposition in Deutschland 2012 - Bericht des Strahlenschutzregisters; Bundesamt für Strahlenschutz / Fachbereich Strahlenschutz und Gesundheit, Salzgitter 2014

Fuhrmann A, Schulze D, Rother U, Vesper M (2003): Digitale transversale Schichtverfahren in der dento-maxillo-fazialen Radiologie. Int J Comput Dent $\underline{6}, 129$ 138

Gernet W, Biffar R, Schwenzer N, Ehrenfeld M: Zahnärztliche Prothetik. 4. Auflage; Thieme Verlag, Stuttgart 2011

Goch I: Vergleich metrischer Genauigkeit von Computertomographie, digitaler Volumentomographie und Orthopantomographie in der modernen Implantologie. Med. Dent. Diss. Düsseldorf 2005 
Gockel F: Eine Untersuchung zur Genauigkeit von dreidimensional, durch CT/DVT geplanten Bohrschablonen für die Insertion von Implantaten in den Kieferknochen. In-vitro Studie an Humankiefern. Med. Diss. Marburg 2009

Gutwald R, Gellrich NC, Schmelzeisen R: Einführung in die zahnärztliche Chirurgie und Implantologie. 2. Auflage; Deutscher Ärzte-Verlag, Köln 2010

Hassfeld S, Streib S, Sahl H, Stratmann U, Fehrentz D, Zoller J (1998): Low- dose computerized tomography of the jaw bone in pre- implantation diagnosis. Limits of dose reduction and accuracy of distance measurements. Mund Kiefer Gesichtschir $\underline{2}$, 188-193

Höhler T: Metrische Genauigkeit der flat panel volumetric computed tomography und verschiedener Orthopantomographien in der präimplantologischen Diagnostik. Med. Dent. Diss. Gießen 2008

Hübinger A: Evaluation dreidimensionaler radiologischer Verfahren zur Darstellung der Mandibula am Humanpräparat. Med. Dent. Diss. Gießen 2008

Klingberg M: Die Abbildungsqualität des digitalen Volumentomographen 3DX im Vergleich zum konventionellen Panoramaschichtgerät. Med. Dent. Diss. Berlin 2008

Koeck B, Wagner W: Implantologie. Elsevier, München 2004

Kreyszig E: Statistische Methoden und ihre Anwendungen. 7. Auflage; Vandenhoeck und Ruprecht, Göttingen 1975

Krockow N: Analyse der Überlebensrate kurzer dentaler Implantate $(\leq 10 \mathrm{~mm})$. Med. Dent. Diss. Düsseldorf 2012

Küker NJ: Metrischer Vergleich von CT, DVT und konventionellen Röntgentechniken in der MKG- Chirurgie. Med. Dent. Diss. Hamburg 2010 
Kyriakou Y, Kolditz D, Langner O, Krause J, Kalender WA (2011): Digitale Volumentomografie (DVT) und Mehrschicht-Spiral-CT (MSCT): eine objektive Untersuchung von Dosis und Bildqualität. Fortschr Röntgenstr 183, 144-153

Lehmann KM, Hellwig E: Zahnärztliche Propädeutik. 12. Auflage; Deutscher ÄrzteVerlag, Köln 2012

Ludlow JB, Ivanovic M (2008): Comparative dosimetry of dental CBCT devices and 64-slice CT for oral and maxillofacial radiology. Oral Surg Oral Med Oral Pathol Oral Radiol Endod 106, 106-114

Ludlow JB, Timothy R, Walker C, Hunter R, Benavides E, Samuelson DB, Scheske MJ (2015): Effective dose of dental CBCT-a meta analysis of published data and additional data for nine CBCT units. Dentomaxillofac Radiol 44(1), 20140197

Mozzo P, Procacci C, Tacconi A, Tinazzi Martini P, Bergamo Andreis IA (1998): A new volumetric $C T$ maschine for dental imaging based on cone-beam technique: preliminary results. European Radiology $\underline{8}, 1558-1564$

Murat S, Kamburoğlu K, Özen T (2012): Accuracy of a newly developed cone-beam computerized tomography- aided surgical guidance system for dental implant placement: an ex vivo study. J Oral Implantol $\underline{38}(6), 706-712$

Nedir R, Bischof M, Briaux JM, Beyer S, Szmukler-Moncler S, Bernard JP (2004): A 7-year life table analysis from a prospective study on ITI implants with special emphasis on the use of short implants. Results from a private practice. Clin Oral Implants Res 15(2), 150-157

Neukam FW, Wichmann M, Wiltfang J: Zahnärztliche Implantologie unter schwierigen Umständen. Georg Thieme Verlag, Stuttgart 2007

Nickenig HJ, Spiekermann H (2006): CT/DVT-basierte implantatprothetische Führungsschablonen vs. Traditionelle Orientierungsschablonen. Z Zahnärztl Impl 22(4), 272-280 
Nitsche T, Menzebach M, Wiltfang J 2012: Indikationen zur implantologischen 3DRöntgendiagnostik und navigationsgestützten Implantologie (S2k). DGZMK vom 8.5.2012 [Zugriff am 10.5.2014]. URL:

http://www.dgzmk.de/zahnaerzte/wissenschaftforschung/leitlinien/details/document/indikationen-zur-implantologischen-3droentgendiagnostik-und-navigationsgestuetzten-implantologie.htm

Park C, Raigrodski AJ, Rosen J, Spiekerman C, London RM (2009): Accuracy of implant placement using precision surgical guides with varying occlusogingival heights: an in vitro study. J Prosthet Dent 101(6), 372-381

Pasler AF: Zahnärztliche Radiologie. 5. Auflage; Georg Thieme Verlag, Stuttgart 2008

Pasler AF, Visser H: Zahnmedizinische Radiologie. 2. Auflage; Georg Thieme Verlag, Stuttgart 2000

Pasler AF, Visser H: Taschenatlas der Zahnärztlichen Radiologie. Georg Thieme Verlag, Stuttgart 2003

Qu XM, Li G, Sanderink GCH, Zhang ZY, Ma XC: Dose reduction of cone beam CT scanning for the entire oral and maxillofacial regions with thyroid collars. Dentomaxillofac Radiol, 41 (5), 373-378

Richter EJ (2005): Implantologie in der Zahnheilkunde. Gemeinsame Stellungnahme der DGZMK und DGI V 3.0, Stand 7/2005. Dtsch Zahnärztl Z 60, 915-941

Román G, Lukas D, Beniashvili R, Schult W : Regionenabhängige Vergrößerungsfaktoren von Panoramaschichtaufnahme in Abhängigkeit von der Patientenpositionierung und ihre Bedeutung für die Implantologie. ResearchPaper, o.Verl. Tübingen, 2003 
Sarment DP, Sukovic P, Clinthorne N (2003): Accuracy of implant placement with stereolithographic surgical guide. Int J Oral Maxillofac Implants $\underline{18}, 571-577$

Schiff T, D'Ambrosio J, Glass BJ, Langlais RP, McDavid, WD (1986): Common positioning and technical errors in panoramic radiography. J Am Dent Assoc 113(3), 422-426

Schulze R, Schalldach F, d'Hoedt B (2000): Auswirkung von Positionierungsfehlern auf Vergrößerungsfaktoren im Unterkiefer bei der digitalen Panoramaschichtaufnahme. Mund Kiefer Gesichts Chir $\underline{4}$ 164-170

Schulze R, Ahlers O, Appel T, Bargholz C, Betz W, Beuer F, Braumann B, Beck J, Deppe H, Edelhoff D, Eickholz P, Haßfeld S, Hirsch C, Hirschfelder U, Jacker-Guhr S, Maager B, Ritter L, Terheyden H (2013): Dentale Digitale Volumentomographie. S2k-Leitlinie. Version Nr. 9 vom 5.8.2013 [Zugriff am 15.6.2014]. URL: http://www.awmf.org/ uploads/tx_szleitlinien/083-005I_S2k_Dentale_ Volumentomographie_2013-10.pdf.

Schwenzer N, Ehrenfeld M: Zahnärztliche Chirurgie. 4. Auflage; Georg Thieme Verlag, Stuttgart New York 2009

Spiekermann H: Implantologie. Georg Thieme Verlag, Stuttgart 1994

Strub JR, Türp JC, Witkowski S, Hürzeler MB, Kern M: Curriculum Prothetik. 3. Auflage; Quintessenz, Berlin 2005

Suckert R.: Okklusionskonzepte. 2. Auflage; Verlag Neuer Merkur, München 1992

Uhl F, Ackermann KL, Dhom GJ, Schmidinger S: Präimplantologische Diagnostik in implantatfrequenten zahnärztlichen, oralchirurgischen und kieferchirurgischen Praxen. Zitiert nach Inhaltsangabe des Vortrages (gehalten 13.10. 2001). 25. Jahrestagung des Arbeitskreises für Forensische Odonto-Stomatologie, Mannheim 11. bis 13.10.2001, Programm und Inhaltsangaben; o. Hrsg., o. Verl., o.O. 2001, 8 (No.3) 
Van Assche N, Van Steenberghe D, Guerrero M E, Hirsch E, Schutyser F, Quirynen $M$, Jacobs $R$ (2007): Accuracy of implant placement based on presurgical planning of three-dimensional conebeam images: a pilot study. J Clin Periodontol 34, 816-821

Weber T: Memorix Zahnmedizin. 3. Auflage; Georg Thieme Verlag, Stuttgart 2010, 307-330

Welander U, McDavid WD, Tronje G, Morris CR (1987): An analysis of different planes within the image layer in rotational panoramic radiography. Dentomaxillofac Radiol $\underline{16}(2), 79-84$

Westendorff C: Experimentelle Untersuchungen zur computergestützten Planung und bilddatengestützten Navigation bei enossalen Implantatlagerpräparationen. Med.

Dent. Diss. Hamburg 2006 
7 Anhang

\subsection{Abkürzungsverzeichnis}

$\begin{array}{ll}\text { CT } & \text { Computertomographie } \\ \text { DVT } & \text { digitaler Volumentomograph } \\ \mathrm{kV} & \text { Kilovolt } \\ \mathrm{I} & \text { Liter } \\ \mathrm{mA} & \text { Milliampere } \\ \mathrm{mSv} & \text { Millisivert } \\ \mu \mathrm{Sv} & \text { Mikrosivert } \\ \mathrm{n} & \text { Anzahl der Versuchsteilnehmer } \\ \text { OPG } & \text { Orthopantomogramm } \\ \mathrm{p} & \text { Wahrscheinlichkeit } \\ \mathrm{t}-\text { Test } & \text { Hypothesentest der t-Verteilung }\end{array}$




\subsection{Auswertungsbögen}

\section{Versuchsbeschreibung (Kugel-, Hülse-, Plättchengruppe)}

Aufgabe: Röntgenbild vermessen

Erklärung des OPG

Kurze Einweisung: Geometriedreieck, digitale Schieblehre

\section{Mit dem Lineal}

Messung für Regio 46:

[Höhe Knochen real] $=\frac{[\text { Höhe Knochen Röntgenbild : }] *[\text { Höhe RöMarker real] }}{\text { [Höhe RöMarker Röntgenbild : }}$

Messung für Regio 47:

[Höhe Knochen real] $=\frac{[\text { Höhe Knochen Röntgenbild : }] \text { [Höhe RöMarker Röntgenbild : }[\text { Höhe RöMarker real] }}{]}$

\section{Mit der Schieblehre}

Messung für Regio 46:

$\left[\right.$ Höhe Knochen real] $=\frac{[\text { Höhe Knochen Röntgenbild : }] *[\text { Höhe RöMarker real] }}{[\text { Höhe RöMarker Röntgenbild : }}$

Messung für Regio 47:

[Höhe Knochen real] $=\frac{[\text { Höhe Knochen Röntgenbild : }] *[\text { Höhe RöMarker real] }}{[\text { Höhe RöM arker Röntgenbild : }}$

Bitte sprechen Sie nicht mit anderen Personen über die Ergebnisse des Versuchs!

Vielen Dank für die Teilnahme! 


\section{Versuchsbeschreibung (Pointgruppe)}

Aufgabe: Röntgenbild vermessen

Erklärung des OPG

Kurze Einweisung: Geometriedreieck, digitale Schieblehre

\section{Mit dem Lineal}

Messung für Regio 46:

[Höhe Knochen real] $=\frac{[\text { Höhe Knochen Röntgenbild : }] * \text { Höhe RöMarker real] }}{[\text { Höhe RöMarker Röntgenbild : }}$

Messung für Regio 47:

[Höhe Knochen real] $=\frac{[\text { Höhe Knochen Röntgenbild : }] *[\text { Höhe RöMarker real] }}{\text { [Höhe RöMarker Röntgenbild : }}$

[Höhe Marker Regio 46: ]

[Höhe Marker Regio 47: ]

\section{Mit der Schieblehre}

Messung für Regio 46:

[Höhe Knochen real] $=\frac{\text { [Höhe Knochen Röntgenbild : }] *[\text { Höhe RöMarker real] }}{\text { [Höhe RöMarker Röntgenbild : }}$

Messung für Regio 47:

[Höhe Knochen real] $=\frac{[\text { Höhe Knochen Röntgenbild : }] *[\text { Höhe RöMarker real] }}{\text { [Höhe RöM arker Röntgenbild : }}$

[Höhe Marker Regio 46: ]

[Höhe Marker Regio 47: ]

Bitte sprechen Sie nicht mit anderen Personen über die Ergebnisse des Versuchs!

Vielen Dank für die Teilnahme! 


\section{Erklärungsbogen für Probanden}

Ziel der Arbeit ist eine Studie zum Vergleich verschiedener Röntgenmarker bei der präoperativen Planung vor einer Implantatinsertion. Dazu wird ein zu diesem Zweck, eigens weiterentwickeltes Röntgenmodell, welches den Unterkiefer mit Zähnen und Canalis Mandibulae darstellt, verwendet.

Vorliegender Fall: Kennedyklasse II mit einseitig verkürzter Zahnreihe rechts, distal des 44. Hier sollen zwei Implantate inseriert werden. Das erste in Regio 46 und das zweite in Regio 47. Da in dieser Region die Gefahr der Verletzung des N. Alveolaris inferior besteht, muss eine sorgfältige präoperative Planung durchgeführt werden.

Erklärung der digitalen Schieblehre: Schieblehre ganz zusammenschieben. Dann einschalten [on]. Gemessen wird die Strecke zwischen den Spitzen der Schieblehre. Das Display zeigt die Distanz in $\mathrm{mm}$ mit zwei Nachkommastellen, die bitte berücksichtigt werden.

\section{Erklärung des OPG:}

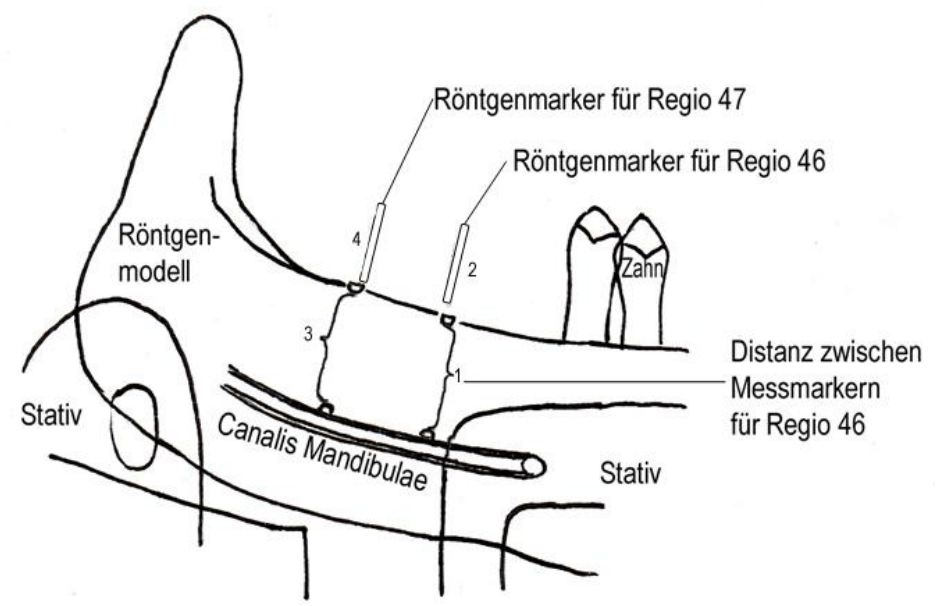

Ihre Aufgabe: Bitte messen Sie mit Hilfe der Schieblehre möglichst genau jeweils die Distanz zwischen den beiden Messmarkern (kürzeste Strecke zwischen den Konvexitäten der Halbkreise) um die röntgenologische Knochenhöhe zu bestimmen. Tragen Sie die beiden Werte in die Gleichung in die leere [ ] ein.

Messung für Regio 46:

$[$ Höhe Knochen real $]=\frac{[}{[\text { Höhe Kugel Röntgenbild }]}$

Messung für Regio 47:

$[$ Höhe Knochen real $]=\frac{[}{[\text { Höhe Kugel Röntgenbild }]}$

Vielen Dank für die Teilnahme! 


\section{OPG/DVT Vergleich}

Knochenhöhe

OPG

Regio 46:_ $\mathrm{mm}$

Regio 47:_ $\mathrm{mm}$

\section{DVT}

Regio 46:_ $\mathrm{mm}$

Regio 47:_ $\mathrm{mm}$ 


\subsection{Rohdaten}

\subsubsection{Versuch 1: Vergleich verschiedener Markersysteme}

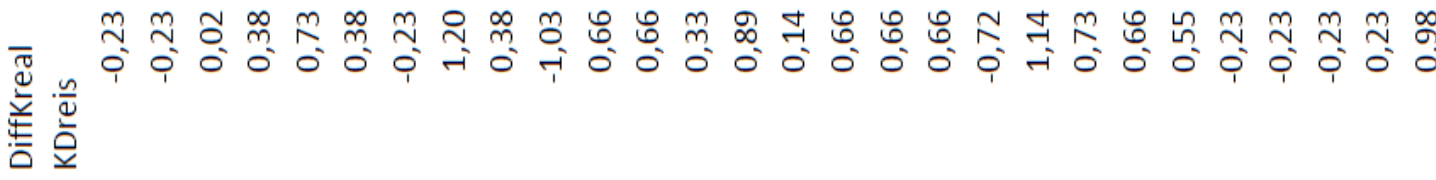

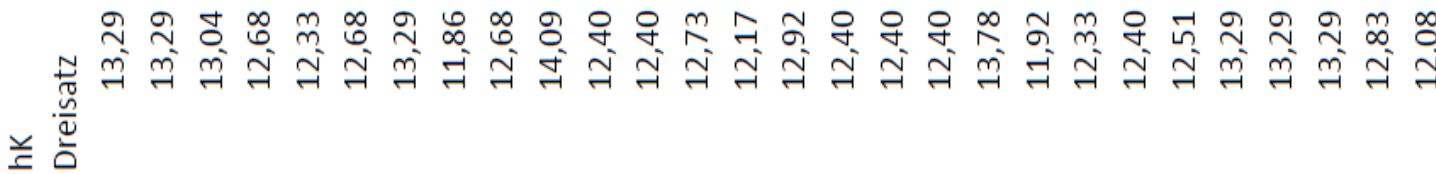

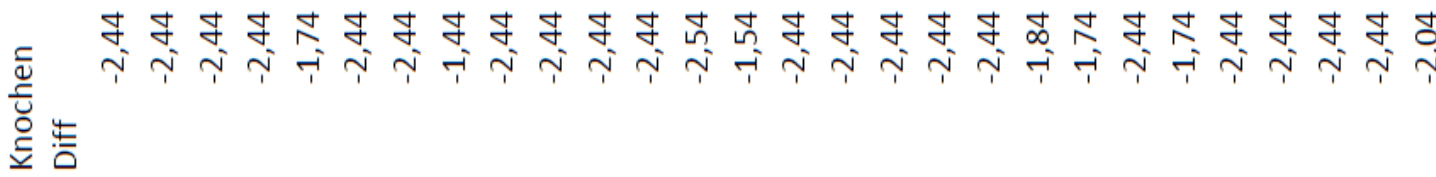

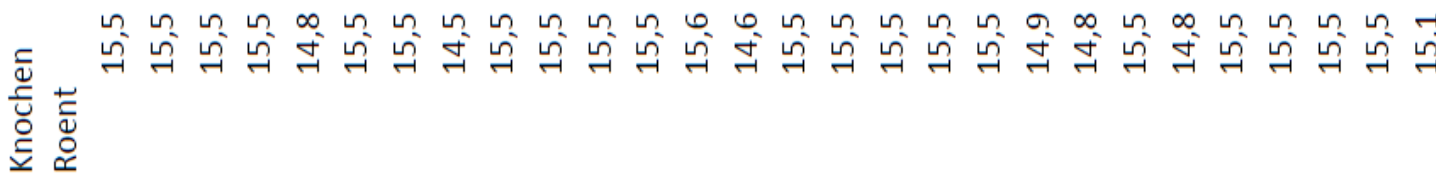
ธ 依

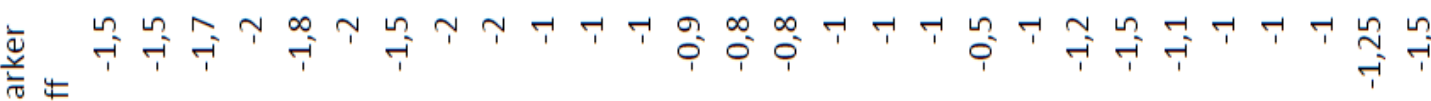
$\sum^{\frac{\pi}{2}}$

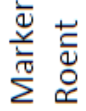

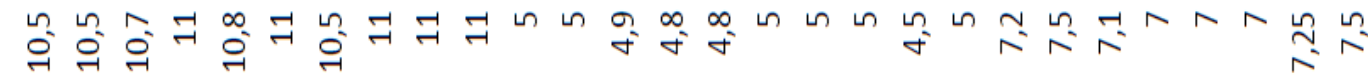

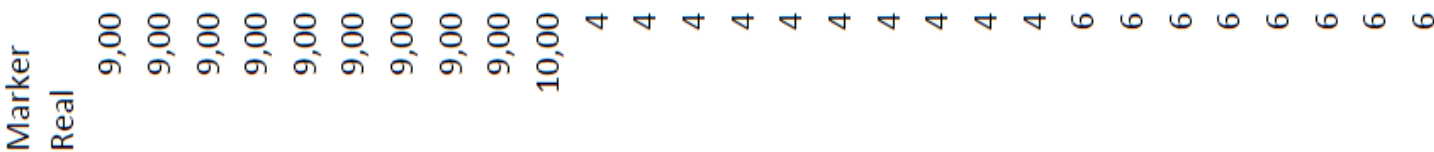

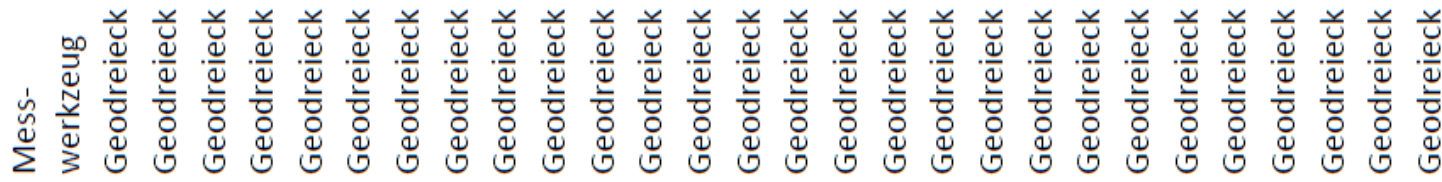

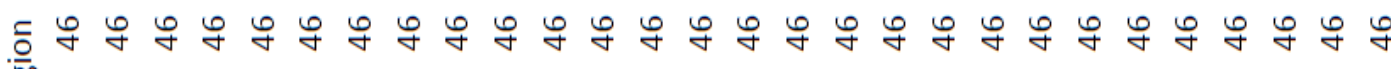

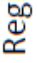

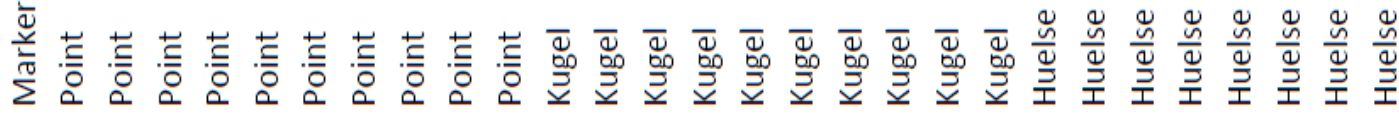

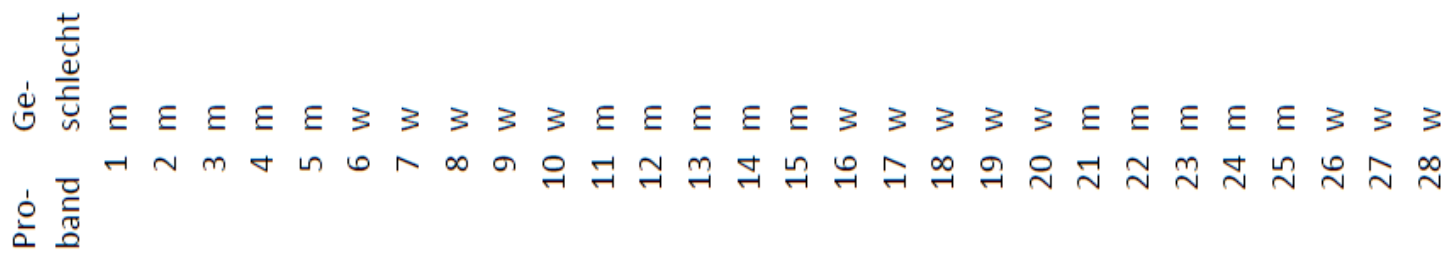




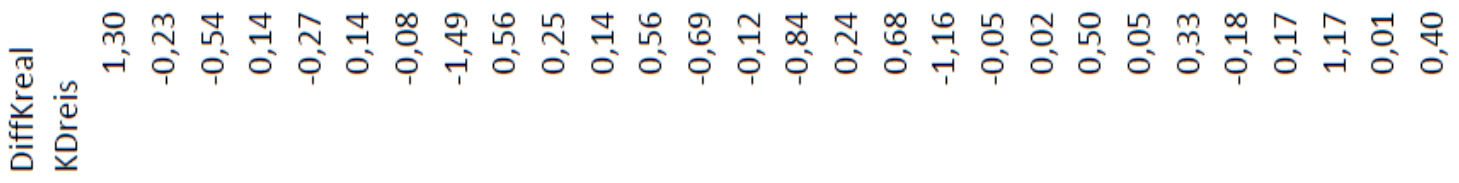

오 우 苞 兰

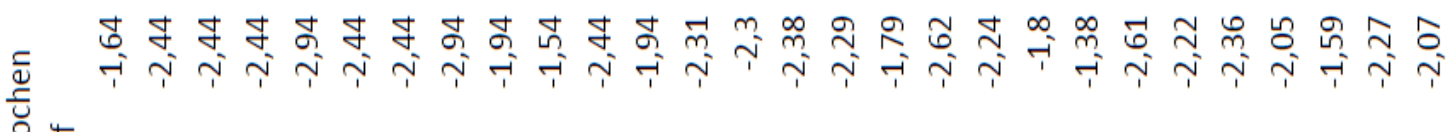
亭

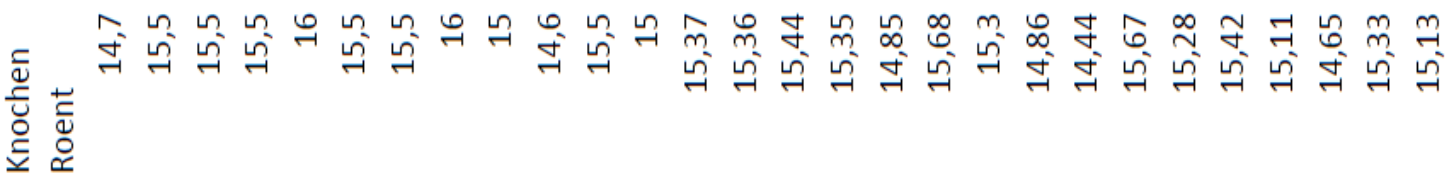

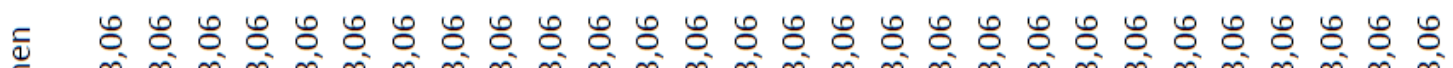
远

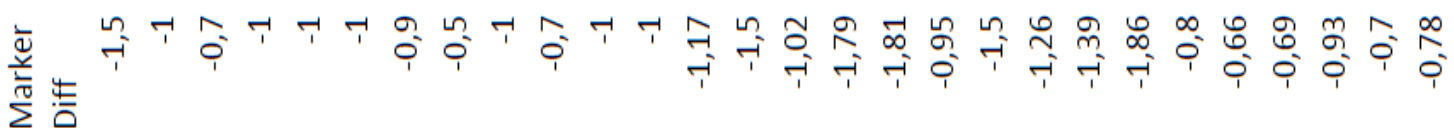

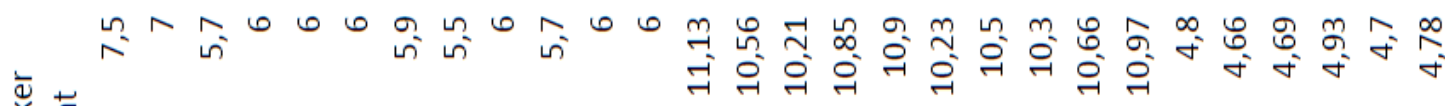

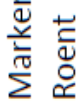

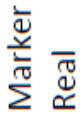

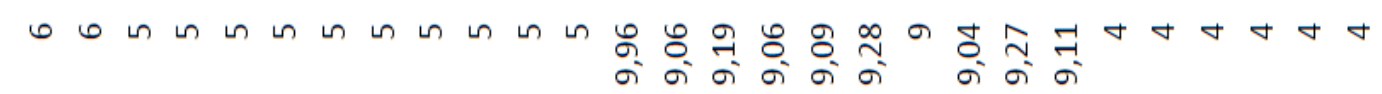

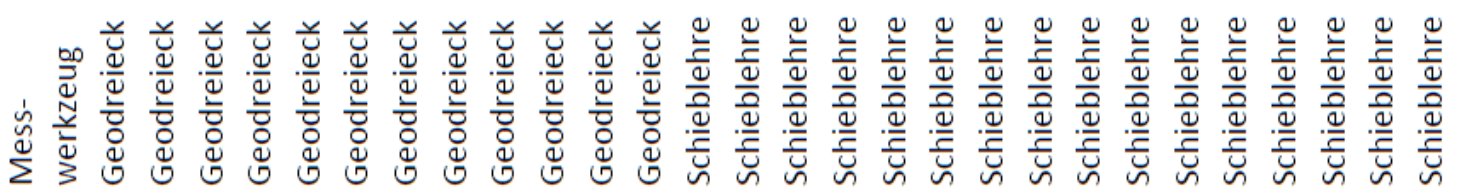
흥 $\stackrel{\infty}{\propto 2}$

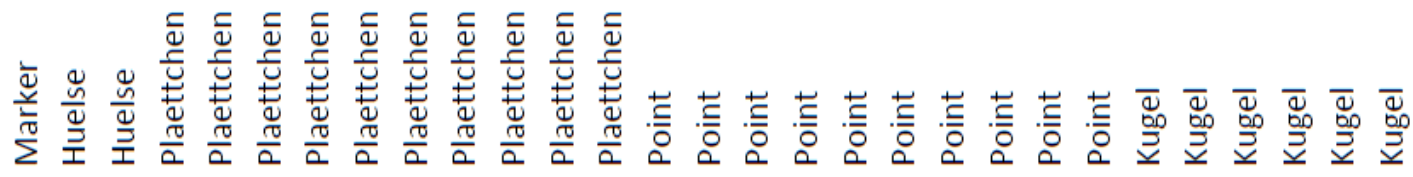

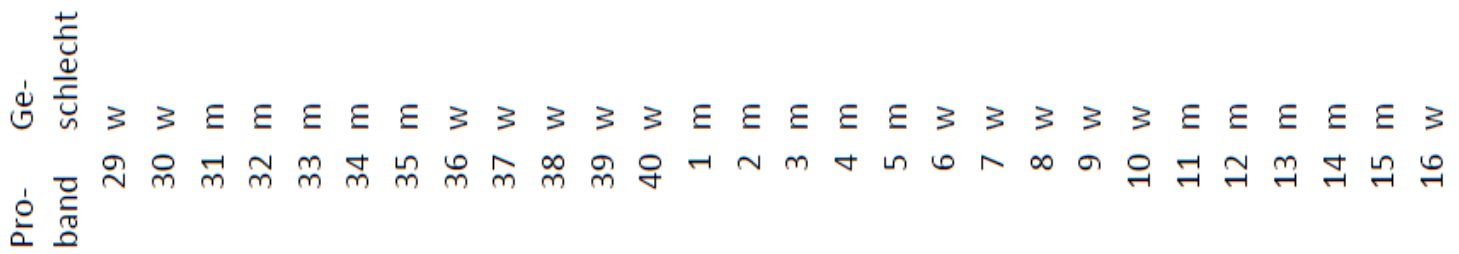




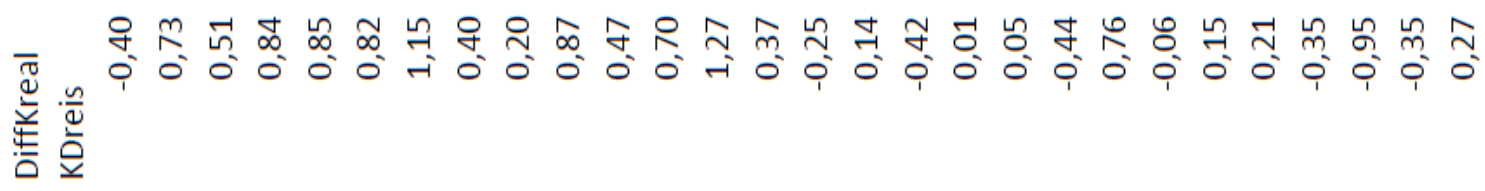

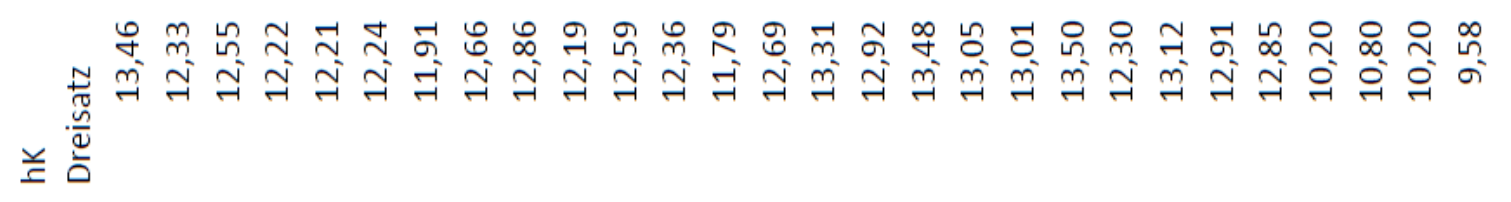

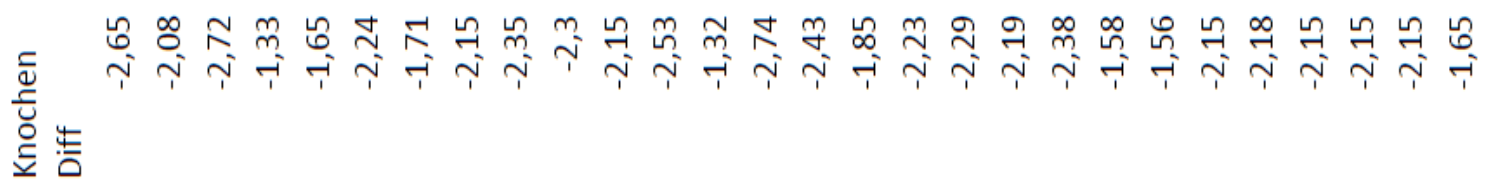

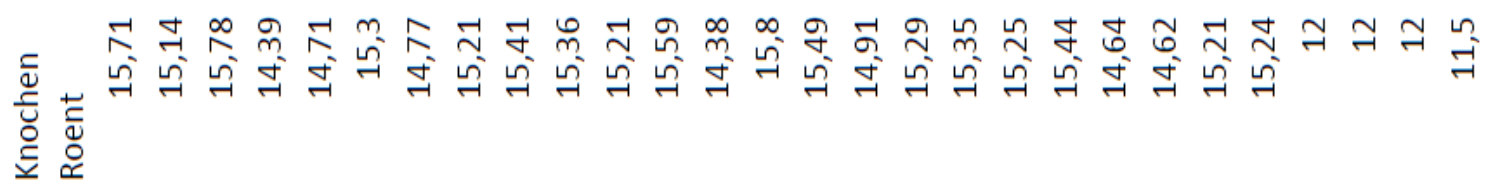

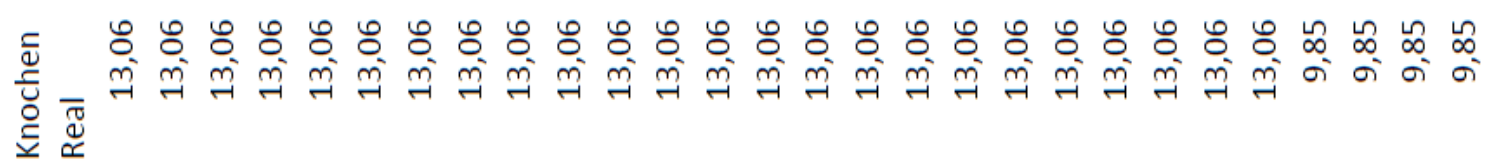

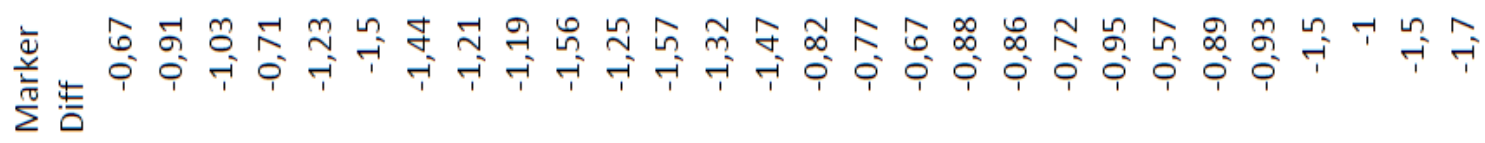
峷言

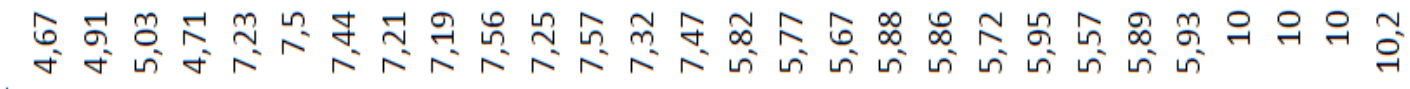
旁京

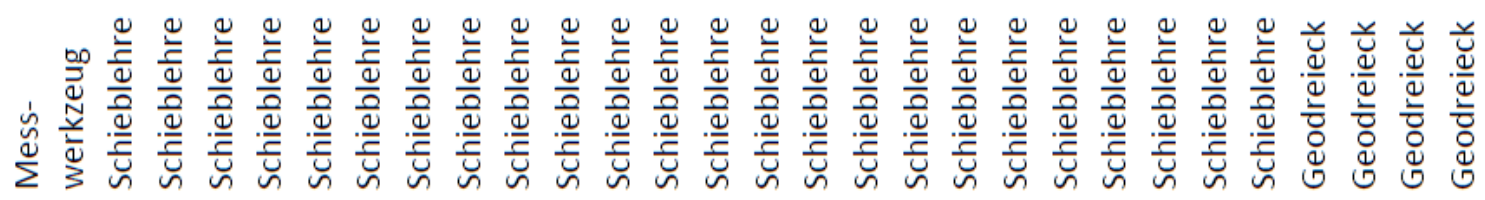

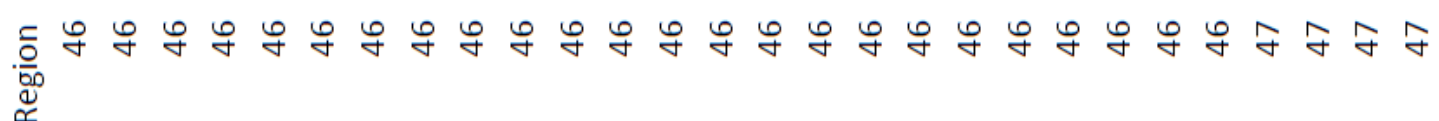

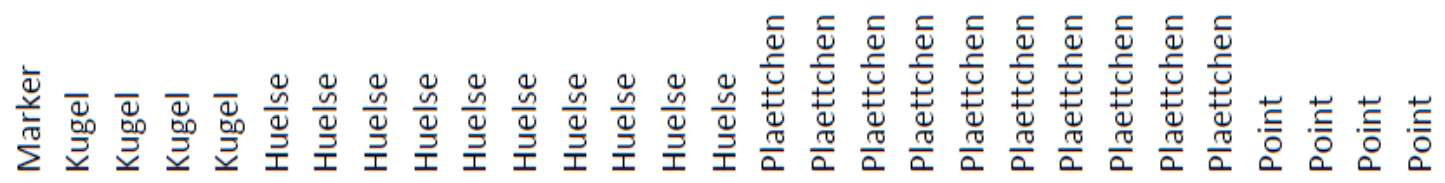

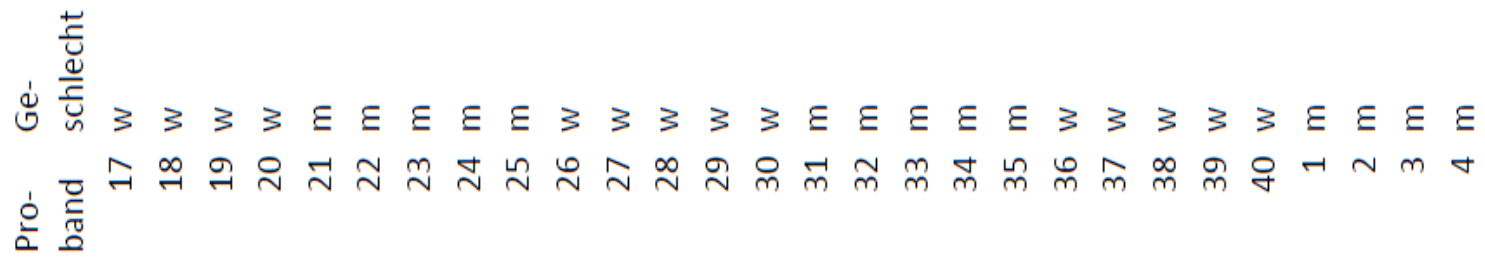




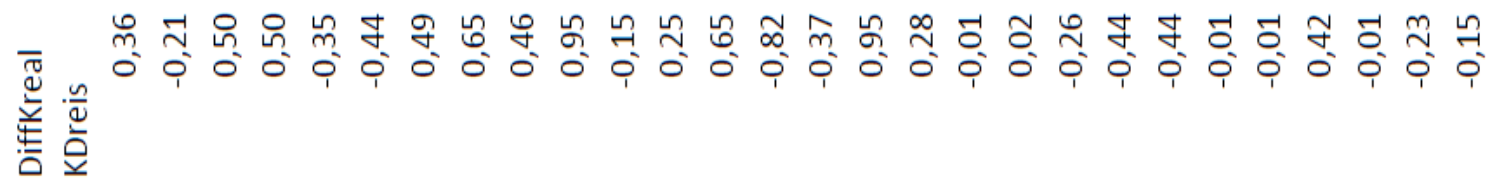

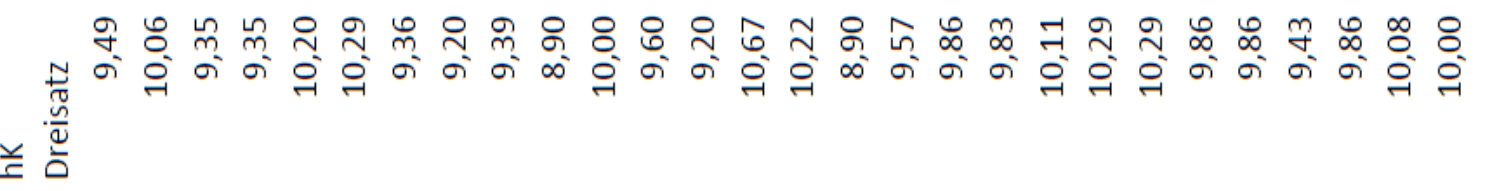

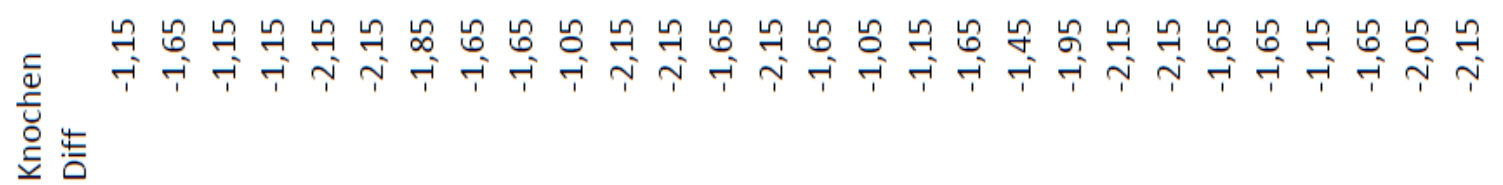

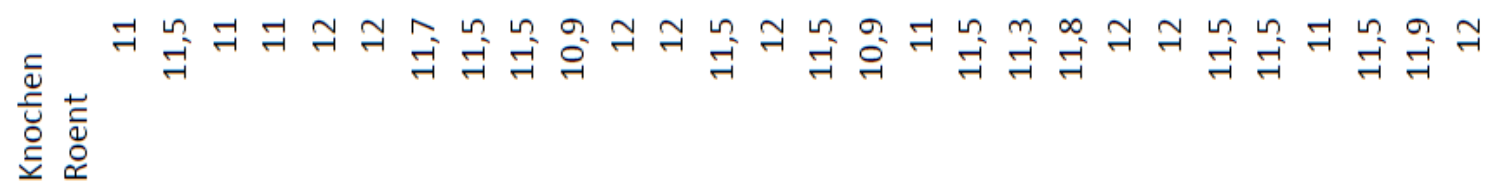

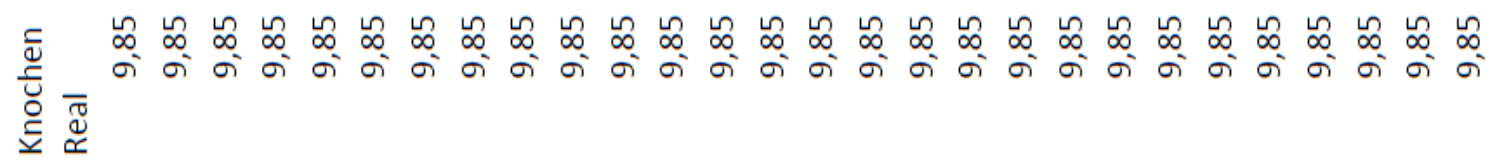

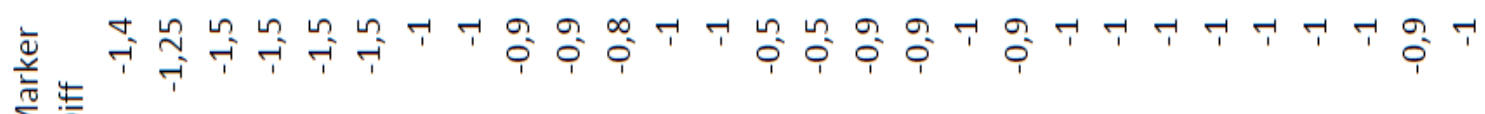

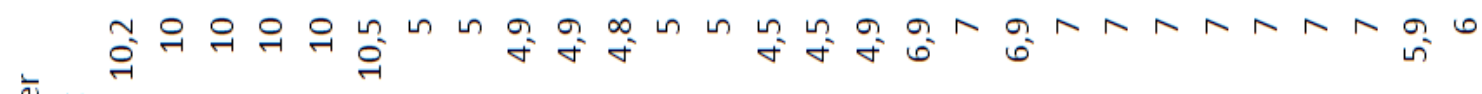
离

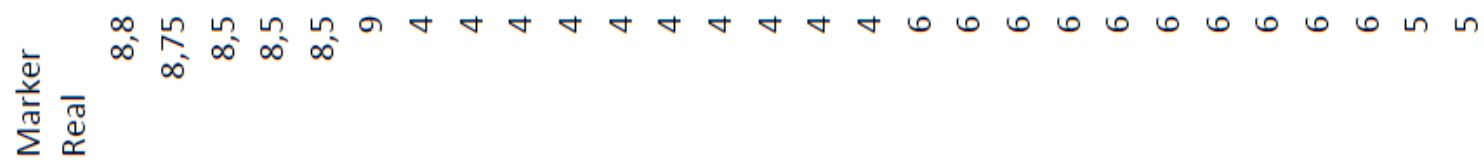

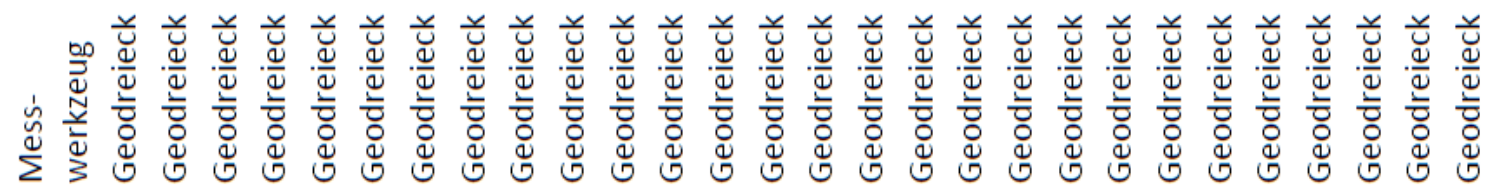
。チ์

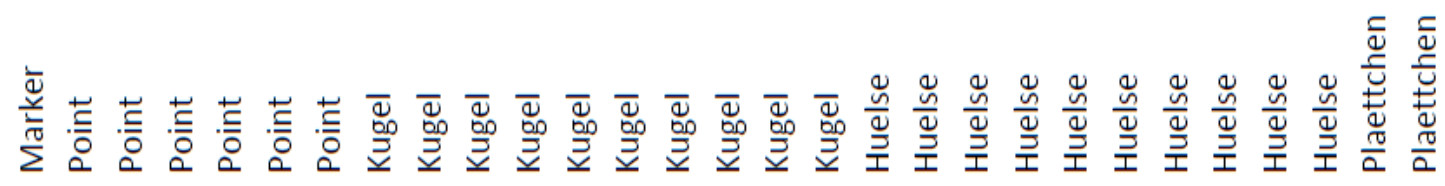
ì $\stackrel{\frac{2}{2}}{\frac{0}{\pi}}$

$\varepsilon 33333 \varepsilon \varepsilon \varepsilon \varepsilon \varepsilon 33333 \varepsilon \varepsilon \varepsilon \varepsilon \varepsilon 33333 \varepsilon \varepsilon$

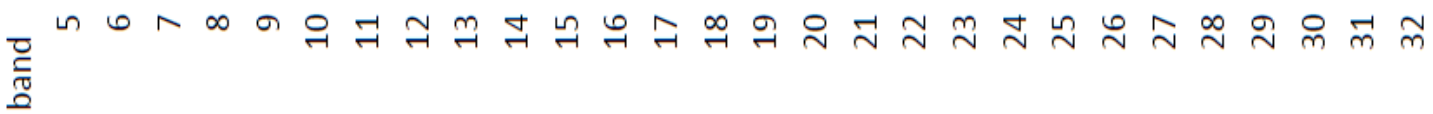




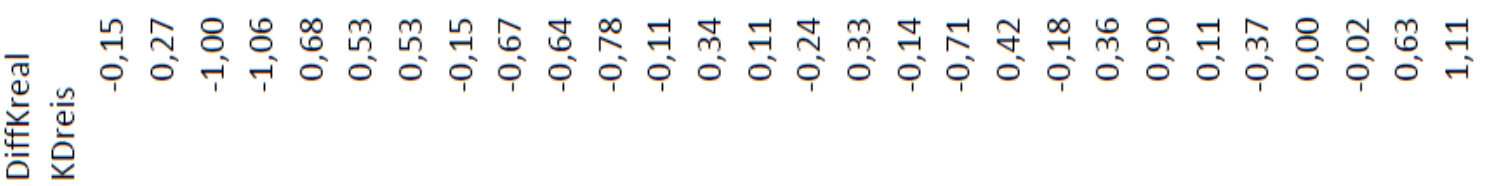

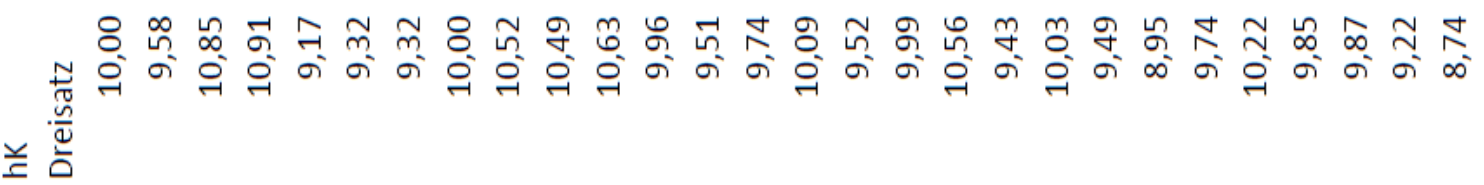

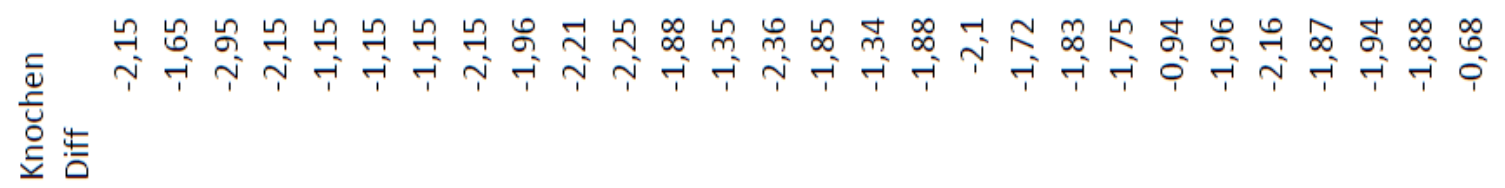

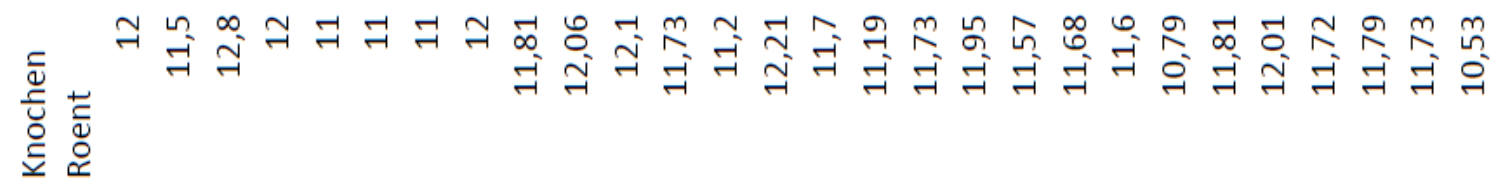

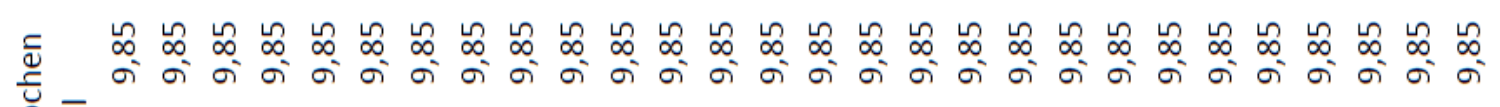
高要

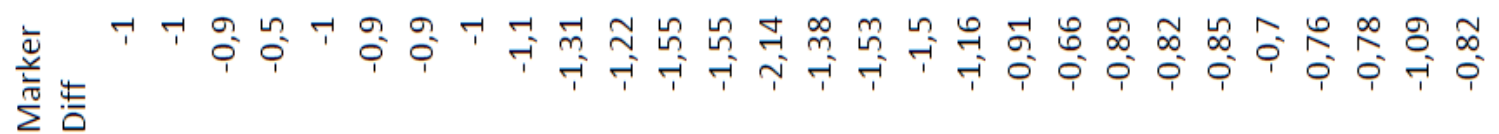

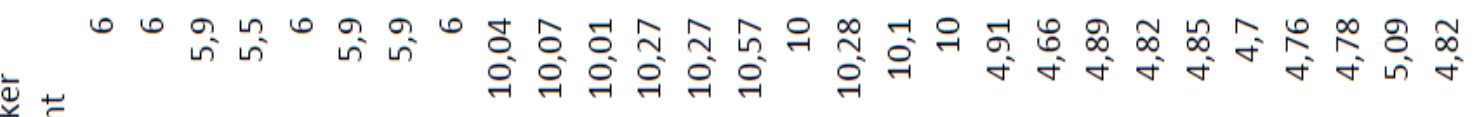
謩言 喜焉

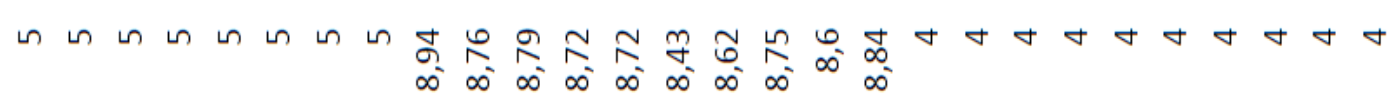

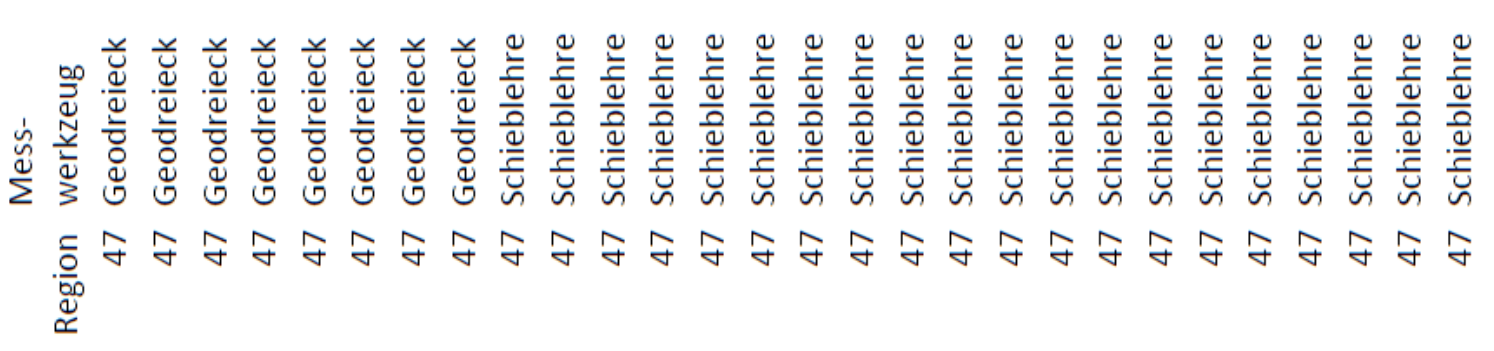

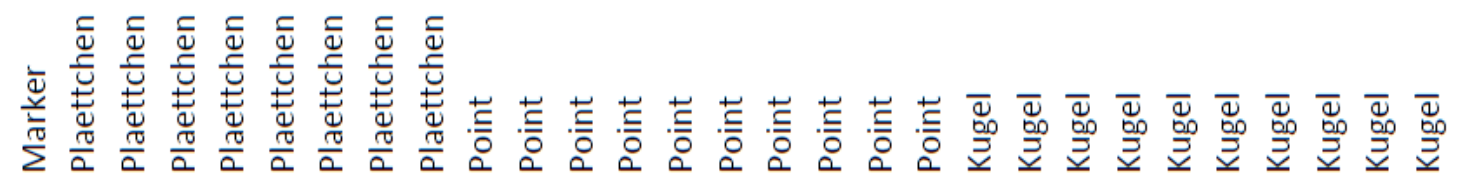
i 인

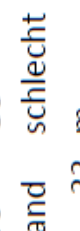

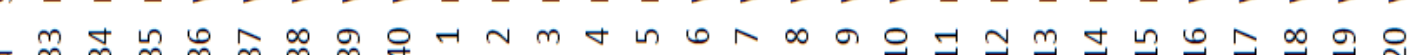
苋 


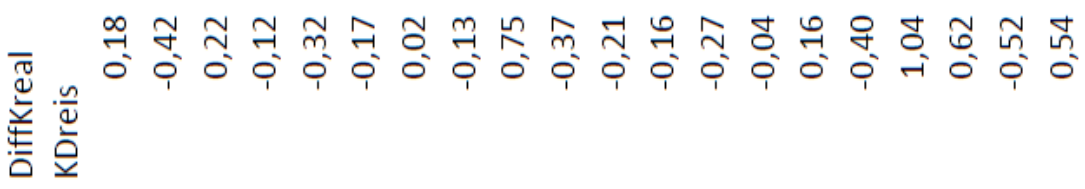

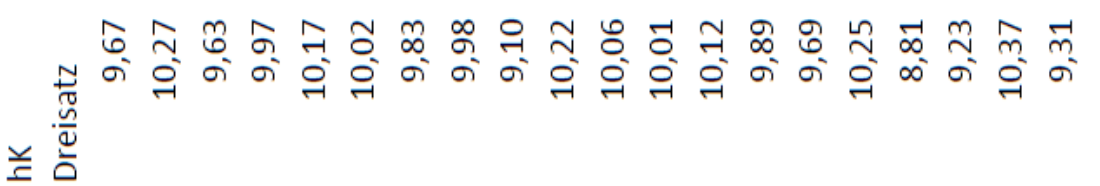

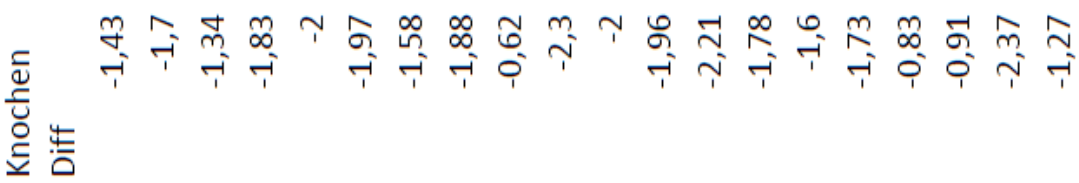

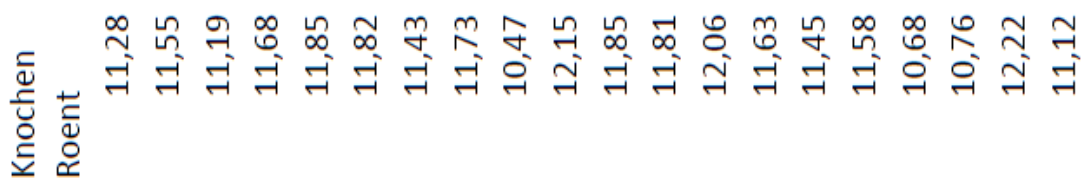

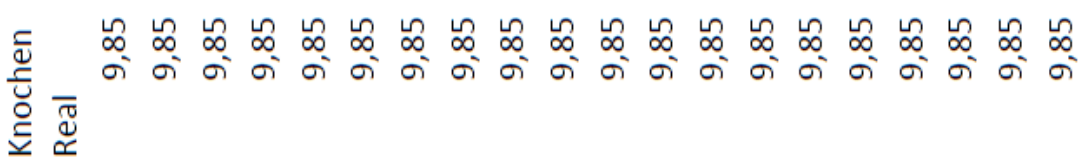

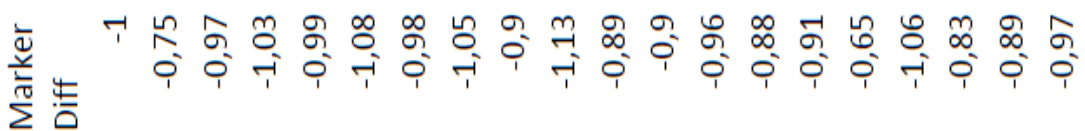

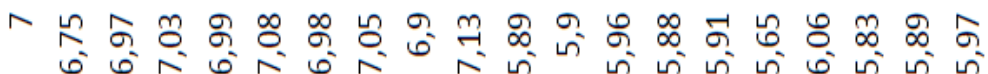

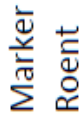
•

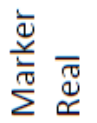

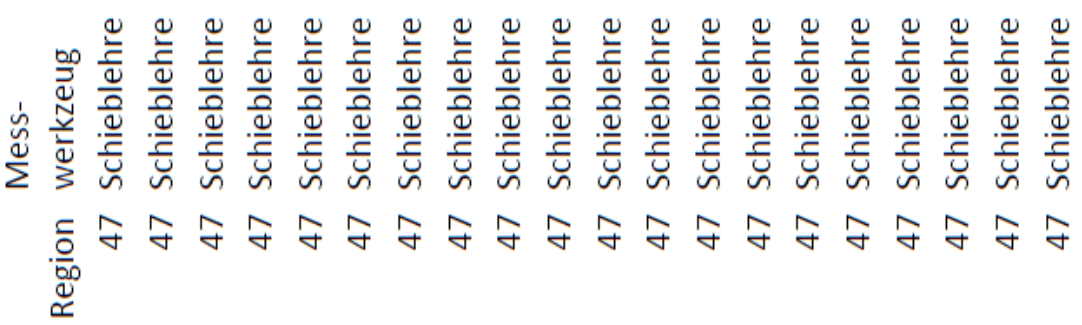

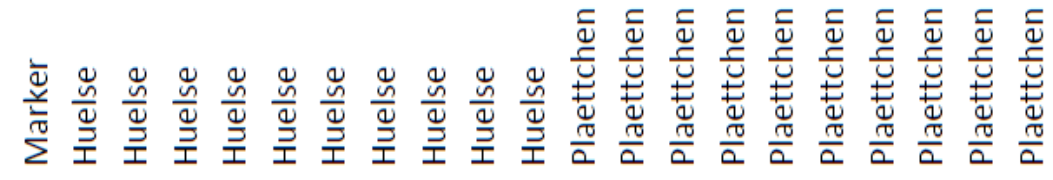

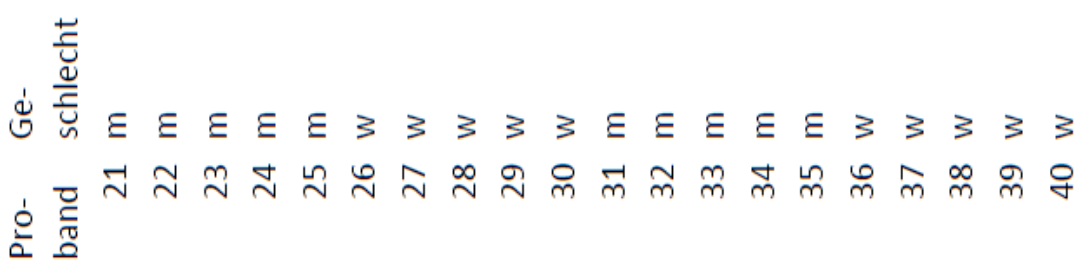




\subsubsection{Versuch 2: DVT vs OPG}

Proband Geschlecht Marker Region Messwerkzeug KnochenRoent KnochenReal KnochenDiff

\begin{tabular}{|c|c|c|c|c|c|c|c|}
\hline 1 & w & Kugel & 46 & OPG X GPlus & 12,59 & 13,06 & 0,47 \\
\hline 2 & $w$ & Kugel & 46 & OPG X GPlus & 12,66 & 13,06 & 0,4 \\
\hline 3 & w & Kugel & 46 & OPG X GPlus & 12,4 & 13,06 & 0,66 \\
\hline 4 & $w$ & Kugel & 46 & OPG X GPlus & 12,57 & 13,06 & 0,49 \\
\hline 5 & $w$ & Kugel & 46 & OPG X GPlus & 12,99 & 13,06 & 0,07 \\
\hline 6 & $\mathrm{~m}$ & Kugel & 46 & OPG X GPlus & 12,49 & 13,06 & 0,57 \\
\hline 7 & $\mathrm{~m}$ & Kugel & 46 & OPG X GPlus & 12,85 & 13,06 & 0,21 \\
\hline 8 & $\mathrm{~m}$ & Kugel & 46 & OPG X GPlus & 12,57 & 13,06 & 0,49 \\
\hline 9 & $\mathrm{~m}$ & Kugel & 46 & OPG X GPlus & 12,58 & 13,06 & 0,48 \\
\hline 10 & $\mathrm{~m}$ & Kugel & 46 & OPG X GPlus & 12,77 & 13,06 & 0,29 \\
\hline 1 & $w$ & Kugel & 46 & NewTom DVT & 13,5 & 13,06 & $-0,44$ \\
\hline 2 & $w$ & Kugel & 46 & NewTom DVT & 13,7 & 13,06 & $-0,64$ \\
\hline 3 & $w$ & Kugel & 46 & NewTom DVT & 13,4 & 13,06 & $-0,34$ \\
\hline 4 & $w$ & Kugel & 46 & NewTom DVT & 13,5 & 13,06 & $-0,44$ \\
\hline 5 & $w$ & Kugel & 46 & NewTom DVT & 14 & 13,06 & $-0,94$ \\
\hline 6 & $\mathrm{~m}$ & Kugel & 46 & NewTom DVT & 13,1 & 13,06 & $-0,04$ \\
\hline 7 & $\mathrm{~m}$ & Kugel & 46 & NewTom DVT & 13,4 & 13,06 & $-0,34$ \\
\hline 8 & $\mathrm{~m}$ & Kugel & 46 & NewTom DVT & 13,4 & 13,06 & $-0,34$ \\
\hline 9 & $\mathrm{~m}$ & Kugel & 46 & NewTom DVT & 13,1 & 13,06 & $-0,04$ \\
\hline 10 & $\mathrm{~m}$ & Kugel & 46 & NewTom DVT & 14 & 13,06 & $-0,94$ \\
\hline 1 & $w$ & Kugel & 47 & OPG X GPlus & 9,37 & 9,85 & 0,48 \\
\hline 2 & $w$ & Kugel & 47 & OPG X GPlus & 9,48 & 9,85 & 0,37 \\
\hline 3 & $w$ & Kugel & 47 & OPG X GPlus & 9,39 & 9,85 & 0,46 \\
\hline 4 & $w$ & Kugel & 47 & OPG X GPlus & 9,49 & 9,85 & 0,36 \\
\hline 5 & $w$ & Kugel & 47 & OPG X GPlus & 9,93 & 9,85 & $-0,08$ \\
\hline 6 & $\mathrm{~m}$ & Kugel & 47 & OPG X GPlus & 9,25 & 9,85 & 0,6 \\
\hline 7 & $\mathrm{~m}$ & Kugel & 47 & OPG X GPlus & 9,48 & 9,85 & 0,37 \\
\hline 8 & $\mathrm{~m}$ & Kugel & 47 & OPG X GPlus & 9,31 & 9,85 & 0,54 \\
\hline 9 & $\mathrm{~m}$ & Kugel & 47 & OPG X GPlus & 9,46 & 9,85 & 0,39 \\
\hline 10 & $\mathrm{~m}$ & Kugel & 47 & OPG X GPlus & 10,22 & 9,85 & $-0,37$ \\
\hline 1 & $w$ & Kugel & 47 & NewTom DVT & 10,2 & 9,85 & $-0,35$ \\
\hline 2 & $w$ & Kugel & 47 & NewTom DVT & 10,1 & 9,85 & $-0,25$ \\
\hline 3 & $w$ & Kugel & 47 & NewTom DVT & 9,6 & 9,85 & 0,25 \\
\hline 4 & $w$ & Kugel & 47 & NewTom DVT & 9,6 & 9,85 & 0,25 \\
\hline 5 & $w$ & Kugel & 47 & NewTom DVT & 10,5 & 9,85 & $-0,65$ \\
\hline 6 & $\mathrm{~m}$ & Kugel & 47 & NewTom DVT & 9,6 & 9,85 & 0,25 \\
\hline 7 & $\mathrm{~m}$ & Kugel & 47 & NewTom DVT & 9,9 & 9,85 & $-0,05$ \\
\hline 8 & $\mathrm{~m}$ & Kugel & 47 & NewTom DVT & 9,9 & 9,85 & $-0,05$ \\
\hline 9 & $\mathrm{~m}$ & Kugel & 47 & NewTom DVT & 9,9 & 9,85 & $-0,05$ \\
\hline 10 & $\mathrm{~m}$ & Kugel & 47 & NewTom DVT & 10,1 & 9,85 & $-0,25$ \\
\hline
\end{tabular}




\section{Danksagung}

Die vorliegende Arbeit entstand unter der Leitung von Herrn Prof. Dr. Dr. Henning Schliephake, Leiter der Klinik für Mund-, Kiefer- und Gesichtschirurgie der Universitätsmedizin Göttingen.

Mein Dank gilt Herrn Dr. Berhard Kirchner für die Einführung in das interessante Thema, die wissenschaftliche Betreuung sowie die Unterstützung bei der Durchführung der Versuche.

Mein besonderer Dank gilt Herrn Prof. Dr. Heiko Visser für die Übernahme der weiteren Betreuung der Dissertation.

Herrn Dr. Klaus Jung aus der Abteilung Medizinische Statistik (Prof. Dr. E. Brunner), gilt mein besonderer Dank für die Unterstützung bei der Versuchsplanung und statistischen Auswertung der Ergebnisse.

Herrn Dr. Thomas Bohne danke ich für die Überlassung der röntgenopaken Unterkiefermodelle, auf deren Grundlage die Modelle für die eigenen Versuche entwickelt werden konnten.

Den MTRAs unserer Röntgenabteilung möchte ich für die freundliche Unterstützung danken; vor allem den leitenden MTRAs Frau Sabine Degenhardt und Frau Tanja Diedrich.

Frau MTA Roswitha Schäfer danke ich für die Einweisung in die Benutzung der Diamantbandsäge zum Bearbeiten der Röntgenmodelle.

Den Zahntechnikern des Zentrums für Zahn-, Mund-, Kieferheilkunde Göttingen unter der Leitung von Herrn Zahntechnikermeister Bernd Weigand danke ich für die fachliche Unterstützung bei der Erstellung der Referenzierungsschablonen. 


\section{Lebenslauf}

Am 8.11.1984 wurde ich, Stephan Lorenz, in Elsterwerda als Sohn der Diplompharmazeutin Andrea Lorenz, geb. Scholz, und des Orgelbaumeisters Heiko Lorenz geboren. Am 11.12.1985 wurde mein Bruder Karsten Lorenz geboren.

Vom 28.8.1997 bis 25.6.2004 besuchte ich das Käthe-Kollwitz-Gymnasium in Wilhelmshaven und erlangte dort die allgemeine Hochschulreife.

Anschließend leistete ich vom 1.7.2004 bis 31.3.2005 meinen Zivildienst in der gastroenterologischen Abteilung des Reinhard-Nieter-Krankenhauses in Wilhelmshaven.

Im April 2005 begann ich das Studium der Zahnmedizin an der Georg-AugustUniversität in Göttingen, an der ich am 18.6.2010 meine Approbation als Zahnarzt erlangte.

Am 12.7.2010 begann ich meine Zeit als Vorbereitungsassistent in der Praxis von Herrn Prof. Dr. Heiko Visser in Oldenburg und bin seit dem 1.1.2013 als angestellter Zahnarzt in derselben Praxis tätig.

Am 20.8.2010 habe ich Merle Kati Lorenz, geb. Weigand, Lehrerin für Grund-, Hauptund Realschule, geheiratet. Am 14.7.2015 kam unsere Tochter Luisa Lorenz auf die Welt. 